\title{
Méthodes expérimentales de l'optique non linéaire
}

\author{
B. Hönerlage
}

Groupe d'Optique Non Linéaire et d'Optoélectronique, Institut de Physique et Chimie des Matériaux de Strasbourg, UMR 7504 du CNRS, ULP, 23 rue du Loess, BP. 20 CR, 67037 Strasbourg cedex, France

\begin{abstract}
Résumé : Dans cet article, nous discutons les différentes techniques et méthodes expérimentales utilisées en spectroscopie optique non linéaire. En particulier, nous considérons les changements d'absorption et de dispersion des matériaux soumis à une forte intensité d'excitation, des mesures de photoluminescence et de gain optique ainsi que du mélange à quatre ondes dans différentes configurations. Dans nos exemples, nous appliquons principalement ces méthodes à des semiconducteurs possédant des zones interdites directes et dipôles actives où des quasi-particules électroniques peuvent être excitées en résonance. Nous obtenons ainsi des informations sur leur durée de vie et de cohérence, les processus de collision et de relaxation d'énergie ainsi que sur les propriétés de transport de ces matériaux.
\end{abstract}

\section{INTRODUCTION : LE CONCEPT DES POLARITONS EXCITONIQUES DANS DES SEMI-CONDUCTEURS MASSIFS}

Dans ce cours, nous discutons principalement la spectroscopie optique des semi-conducteurs soumis à une forte intensité d'excitation lumineuse résonante. Nous introduisons donc tout d'abord le concept du polariton excitonique. Il décrit la relation de dispersion du champ électromagnétique et le lie avec la fonction diélectrique complexe.

Nous considérons l'équation de Maxwell macroscopique qui relie le champ électrique $\vec{E}$ (qui dépend de sa coordonnée dans l'espace $\vec{r}$ et du temps t) et la polarisation $\vec{P}$ avec le déplacement électrique $\vec{D}$ :

$$
\vec{D}(\vec{r}, t)=\varepsilon_{0} \vec{E}(\vec{r}, t)+\vec{P}(\vec{r}, t)
$$

$\varepsilon_{0}$ est la permittivité du vide. Les composantes $\mathrm{P}_{\mathrm{i}}, \mathrm{i}=(\mathrm{x}, \mathrm{y}, \mathrm{z})$ du vecteur $\overrightarrow{\boldsymbol{P}}$, sont reliées à celles du champ électrique par le tenseur de la susceptibilité $\chi\left(\vec{r}, \overrightarrow{\left.r^{\prime}, t, t^{\prime}\right)}\right.$ :

$$
P_{i}(\vec{r}, t)=\varepsilon_{0} \sum_{j} \iint \chi_{i j}\left(\vec{r}, \vec{r}^{\prime}, t, t^{\prime}\right) E_{j}\left(\vec{r}^{\prime}, t\right) d^{3} r^{\prime} d t^{\prime}
$$


où $\chi_{\mathrm{ij}}$ sont les composantes du tenseur $\chi$. Si le système est homogène dans l'espace et en temps, le tenseur de susceptibilité ne dépend que de la différence des coordonnées spatiales et temporelles, et il vient:

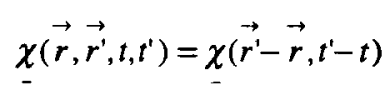

Dans la suite, nous nous limitons à cette situation, ce qui implique que nous ne pouvons pas décrire des effets dus aux surfaces ou à des variations temporelles très rapides. $\vec{D}, \vec{E}$ et $\vec{P}$ possèdent une transformée de Fourier et on peut écrire l'équation (1) sous la forme :

$$
\begin{aligned}
D_{i}(\vec{r}, t)=\iint D_{i}(\vec{k}, \omega) e^{i(\vec{k} \vec{r}-\omega . t)} d^{3} k d \omega=\varepsilon_{0} \iint \vec{E}_{i}(\vec{k}, \omega) e^{i(\vec{k} \vec{r}-\omega \cdot t)} d^{3} k d \omega+ \\
\quad+\varepsilon_{0} \sum_{j} \iint \chi_{i j}\left(\overrightarrow{r^{\prime}}-\vec{r}, t^{\prime}-t\right) E_{j}(\vec{k}, \omega) e^{i\left(\vec{k} \vec{r}^{\prime}-\omega \cdot t^{\prime}\right)} d^{3} k d \omega d^{3} r^{\prime} d t^{\prime}
\end{aligned}
$$

L'équation (4) étant valable à chaque instant $t$ et en tout point $\vec{r}$, on peut appliquer le théorème de Fourier et écrire :

$$
D_{i}(\vec{k}, \omega) e^{i(\vec{k} \vec{r}-\omega x)}=\varepsilon_{0} E_{i}(\vec{k}, \omega) e^{i(\vec{k} \vec{r}-\alpha r)}+\sum_{j} \varepsilon_{0} E_{j}(\vec{k}, \omega) \iint \chi_{i j}\left(\overrightarrow{r^{\prime}}-\vec{r}, t^{\prime}-t\right) e^{i\left(\vec{k} \vec{r}^{\prime}-\omega r^{\prime}\right)} d^{3} r^{\prime} d t^{\prime}
$$

En divisant l'équation (5) par $e^{i(\vec{k} \vec{r}-\omega x)}$ et en introduisant les nouvelles variables $\vec{\rho}=\overrightarrow{r^{\prime}}-\vec{r}$ et $\tau=t^{\prime}-t$, on obtient finalement :

$$
D_{i}(\vec{k}, \omega)=\varepsilon_{0} E_{i}(\vec{k}, \omega)+\varepsilon_{0} \sum_{j} \chi_{i j}(\vec{k}, \omega) E_{j}(\vec{k}, \omega)
$$

où $\chi_{i j}(\vec{k}, \omega)$ est la transformée de Fourier de la susceptibilité :

$$
\chi_{i j}(\vec{k}, \omega)=\iint \chi_{i j}(\vec{\rho}, \tau) e^{i(\bar{k} \vec{\rho}-\omega \tau)} d^{3} \rho d \tau
$$

En utilisant la notation vectorielle, on peut écrire l'équation (6) sous la forme :

$$
\vec{D}(\vec{k}, \omega)=\varepsilon_{0}[1+\underline{x}(\vec{k}, \omega)] \vec{E}(\vec{k}, \omega)
$$

où la fonction diélectrique $\varepsilon(\vec{k}, \omega)$ est donnée par :

$$
\varepsilon(\vec{k}, \omega)=1+\chi \underline{\underline{k}}, \omega)
$$

On peut remarquer que $\varepsilon(\vec{k}, \omega)$ est une constante si la réponse du milieu est locale, c'est-àdire si $\chi(\rho, \tau)=\delta(\rho) \delta(\tau) \chi_{0}$. Dans notre cas, la réponse est non locale, mais comme nous le verrons dans la suite, $\vec{k}$ et $\omega$ ne sont pas des variables indépendantes l'une de l'autre. Elles sont connectées par la relation de dispersion due à la nature ondulatoire du champ électromagnétique. 
Comme il a été discuté dans le chapitre "Introduction à l'optique non linéaire" de ce cours, le modèle le plus simple pour discuter la réponse linéaire d'un milieu diélectrique consiste à considérer les électrons comme des oscillateurs harmoniques, soumis à un champ électromagnétique. Ce champ induit la polarisation atomique macroscopique $\vec{P}_{A}$ qui est gouvernée par l'équation différentielle :

$$
\frac{\partial^{2} \overrightarrow{P_{A}}(t)}{\partial t^{2}}+\omega_{0}^{2} \overrightarrow{P_{A}(t)}=\frac{e N}{m} \vec{E}(t)
$$

où $\omega_{0}, \mathrm{e}, \mathrm{m}, \mathrm{N}$ sont respectivement la fréquence de résonance, la charge élémentaire, la masse et le nombre des électrons qui sont liés harmoniquement. À l'aide des équations de Maxwell, en éliminant le champ magnétique, on trouve, pour un système diélectrique sans sources (électrons libres), l'équation différentielle du champ électromagnétique propagatoire :

$$
\Delta \vec{E}-\mu_{0} \varepsilon_{0} \frac{\partial^{2} \vec{E}}{\partial t^{2}}=\mu_{0} \frac{\partial^{2} \vec{P}_{A}}{\partial t^{2}}
$$

où $\mu_{0}$ est la perméabilité du vide.

Les deux équations différentielles (10) et (11) peuvent être résolues en termes d'ondes planes. En éliminant $\vec{P}_{A}, \vec{E}$ prend la forme :

$$
\vec{E}=\left(0, E_{y}, 0\right)
$$

avec $\mathrm{E}_{\mathrm{y}}(\mathrm{k}, \omega)=\mathrm{A} \mathrm{e}^{\mathrm{i}(\mathbf{k z -}-\boldsymbol{\omega t})}$ si on suppose que le champ $\vec{E}$ est polarisé linéairement suivant $\mathrm{y}$ et que l'onde se propage parallèlement à $z$. De plus, $\omega$ et $\mathbf{k}$ doivent obéir à la relation de dispersion:

$$
\frac{k^{2}}{\mu_{0} \varepsilon_{0} \omega^{2}}=1+\frac{\alpha}{\omega_{0}^{2}-\omega^{2}}
$$

ainsi que :

$$
\frac{k^{2}}{\mu_{0} \varepsilon_{0} \omega^{2}}=\varepsilon_{\perp}(k, \omega)
$$

où $\frac{1}{\sqrt{\mu_{0} \varepsilon_{0}}}=c$ est la vitesse de la lumière dans le vide, $\varepsilon_{\perp}(k, \omega)$ est la fonction diélectrique transverse et $\alpha=\mathrm{Ne}^{2} / \mathrm{m}$ est la polarisabilité macroscopique du système. $\mathrm{D}^{\circ}$ autre part, les équations (11) et (12) donnent aussi la condition :

$$
0=\omega^{2} \varepsilon_{l l}(k, \omega)
$$

Pour $\omega \neq 0$, l'équation (15) définit la condition pour l'existence d'un état propre longitudinal à la fréquence $\omega_{\mathrm{L}}$ :

$$
\varepsilon_{/ \prime}\left(k, \omega_{L}\right)=0
$$

Les plasmons ainsi que les excitons longitudinaux correspondent à une excitation 
collective avec une polarisation d'un métal ou semi-conducteur parallèle à leur direction de propagation.

L'équation 13 veut dire que si nous excitons avec une onde électromagnétique des oscillateurs harmoniques (ici des électrons que nous déplaçons par rapport à leur barycentre de charge) c'est l'ensemble du champ et de l'onde de polarisation qui se propage. Nous introduisons donc la notation des polaritons pour ces nouvelles quasi-particules. La vitesse de groupe de ce paquet d'ondes (donné par d $\omega / \mathrm{dk}$ ) est plus petite que celle du champ électromagnétique dans le vide. Elle diminue d'autant plus que l'on s'approche de la fréquence propre des oscillateurs $\omega_{0}$. Ceci est bien visible sur la figure 1 qui montre la forme typique de la relation de dispersion (équation 13).

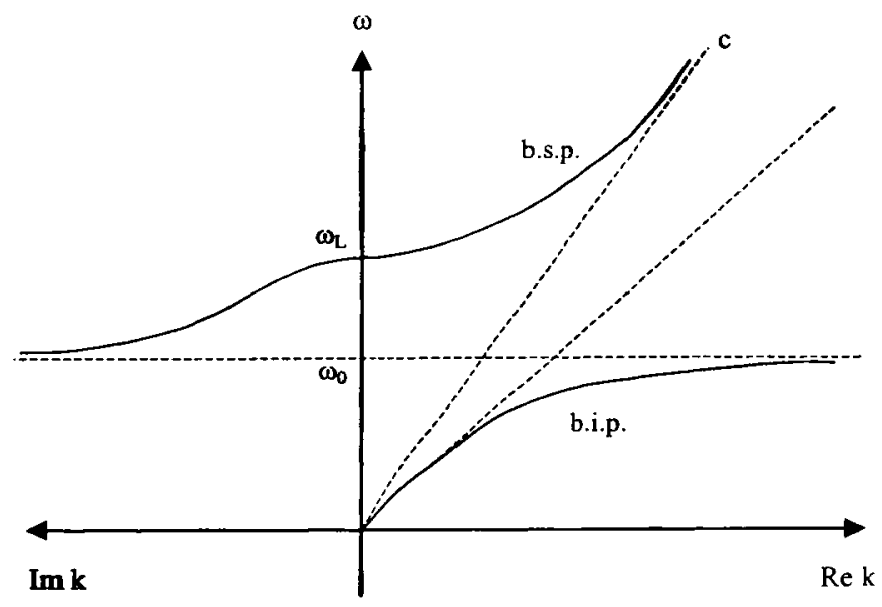

Figure 1 : Relation de dispersion E (k) pour des oscillateurs isolés.

On remarque bien que si des quasi-particules avec leur fréquence de résonance $\omega_{0}$ sont excitées par le champ électromagnétique, la dispersion possède deux branches réelles que nous appelons branche "supérieure" et "inférieure de polariton" (b.s.p. et b.i.p.). Ces deux branches sont séparées par une zone interdite entre $\omega_{0}$ et $\omega_{\mathrm{L}}$ où la solution de l'équation 13 est purement imaginaire. Entre $\omega_{\mathrm{L}}$ et $\omega_{0}$ n'existe donc pas de solution propagatoire du système composé du champ électromagnétique couplé aux oscillateurs harmoniques. Cette situation est bien connue en physique : les solutions propres d'un Hamiltonien décrivant deux états qui possèdent la même symétrie qui sont couplés ne peuvent pas être dégénérés (level anticrossing). Ici, les deux états sont représentés par le champ électromagnétique et les oscillateurs qui peuvent être des phonons (phonons-polaritons) ou, dans notre cas, des excitons (excitons-polaritons).

La dispersion de la b.s.p. commence à $k=0$ où $\omega=\omega_{\mathrm{L}}$, c'est-à-dire que la branche transverse de polariton est ici dégénérée avec l'onde de polarisation longitudinale. Ceci est une conséquence de la symétrie du problème : pour $k \neq 0$, on peut définir une polarisation perpendiculaire à la direction de propagation. $\grave{A} \mathrm{k}=0$ ceci n'est pas possible (il n'existe pas de direction préférentielle) et les fréquences des oscillateurs longitudinaux et transverses doivent être les mêmes. 
Nous allons maintenant discuter la dispersion qualitativement, en introduisant quelques modifications au modèle simple.

Tout d'abord si l'on calcule la relation de dispersion en mécanique quantique à partir d'un modèle analogue de celui présenté ici, on obtient aussi le résultat donné par l'équation (13) Dans ce modèle, on élimine le couplage fort champ-matière par une transformation de Bogoliubov. La polarisabilité atomique macroscopique $\alpha$ est dans ce cas remplacée par la force d'oscillateur $\beta_{\mathrm{j}}$ qui est proportionnelle au carré de l'élément de transition entre l'état fondamental " 0 " et l'état final “ $j$ " [1,2] sous l'action du champ périodique. Si le système possède plusieurs résonances dipolactives isolées, il faut sommer sur ces différentes contributions. De plus, on peut considérer le cas où les oscillateurs harmoniques sont couplés entre eux et sont amortis par un terme de forme $-\gamma, \dot{\vec{P}}_{A}$ dans l'équation différentielle (10). Le résultat est donné par l'expression :

$$
\varepsilon(k, \omega)=\frac{c^{2} k^{2}}{\omega^{2}}=1+\sum_{j} \frac{\beta_{j} \omega_{0}^{j^{2}}(k=0)}{\omega_{0}^{j^{2}}(k)-\omega^{2}-i \omega \gamma_{j}}
$$

où $\varepsilon$ est une fonction complexe. De plus, dans le cas des excitons polaritons, on montre $[1,2]$ que :

$$
\beta_{j}=\frac{\omega_{L}^{j}(k=0)^{2}-\omega_{0}^{j}(k=0)^{2}}{\omega_{0}^{j}(k=0)^{2}}
$$

est donné par l'interaction d'échange non analytique entre électron et trou. $\omega_{0}^{j}(k)$ donne la dispersion des excitons qui est habituellement considérée dans l'approximation de la masse effective (donc avec " dispersion spatiale").

$$
\omega_{0}^{j}(k)=\omega_{0}^{j}+\hbar^{2} k^{2} / 2 M
$$

où $\mathbf{M}$ est la masse effective du mouvement du centre de masse de l'exciton et $\hbar \mathrm{k}$ sa quantité de mouvement. Si une des résonances "j" est très loin et bien isolée des autres, on peut simplifier l'équation (17) pour $\omega$ autour de $\omega_{0}^{j}$ et on obtient dans cette "approximation à un oscillateur" :

$$
\varepsilon(k, \omega)=\frac{c^{2} k^{2}}{\omega^{2}}=\varepsilon_{b}\left(1+\frac{\beta \omega_{0}^{2}}{\omega_{0}^{2}-\omega^{2}-i \gamma \omega}\right)
$$

où nous avons considéré l'indice $\mathrm{j}$ comme étant muet et où $\varepsilon_{\mathrm{b}}$ désigne la constahte diélectrique dit du "background", terme simulant l'influence de toutes les autres transitions qui sont permises et qui sont excitées virtuellement par le champ électromagnétique. Pour $\gamma=0$, l'équation (17) possède une solution analytique de forme :

$$
E(Q)=\frac{1}{\sqrt{2}}\left(A \pm \sqrt{A^{2}-4 B^{2}}\right)^{1 / 2}
$$

avec $A=\hbar^{2} c^{2} Q^{2} / \varepsilon_{b}+\hbar^{2} \omega_{0}^{2}(Q)(1+\beta), B^{2}=\hbar^{4} c^{2} Q^{2} \omega_{0}^{2}(Q) / \varepsilon_{b}, \mathrm{E}_{\mathrm{L}}=\hbar \omega_{\mathrm{L}}$ et $\mathrm{E}_{0}=\hbar \omega_{0}$ $\mathrm{Q}$ est la quantité de mouvement (dans ce cas réel) de l'exciton-polariton. Les solutions de l'équation (20) sont présentées sur la figure 2 


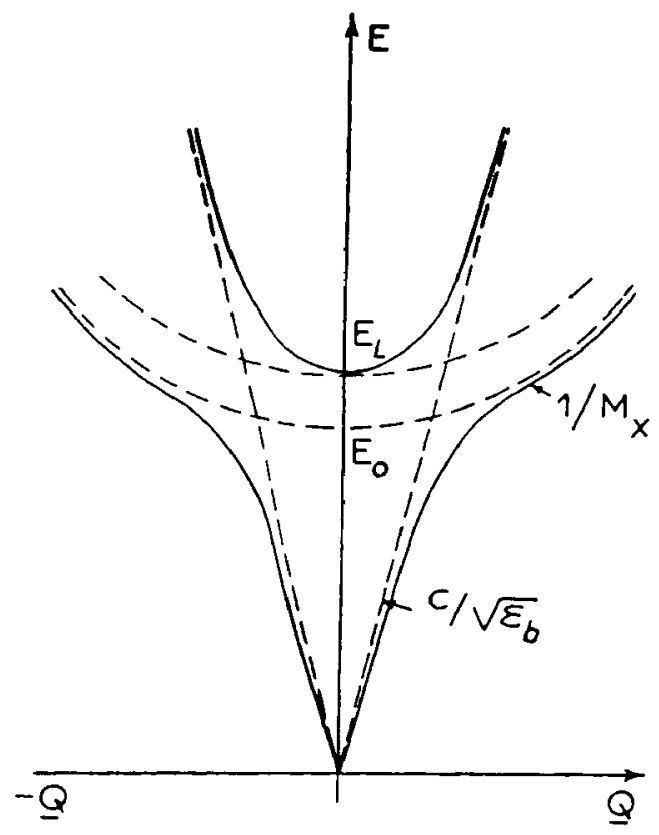

Figure 2 : Relation de dispersion des polaritons excitoniques dans l'approximation d'un oscillateur avec dispersion spatiale et sans amortissement.

Si l'on compare la figure 2 avec la figure 1 , on remarque que pour $E<\hbar \omega_{\mathrm{L}}$ il existe toujours une solution réelle. Pour $\mathrm{E}>\hbar \omega_{\mathrm{L}}$, deux solutions réelles (propagatoires) sont présentes qui correspondent à des paquets d'ondes de caractère (excitoniques ou photoniques) et de vitesse de groupes différents. Hors de la résonance, la b.s.p. s'approche d'une manière asymptotique d'une droite de pente $c / \sqrt{\varepsilon_{b}}$. Autour de $\mathrm{E} \sim \mathrm{E}_{0}$, la densité d'état de la b.i.p. change rapidement et les quasi-particules ont un caractère excitonique pour $E \gg E_{0}$ et photonique pour $\mathrm{E} \ll \mathrm{E}_{0}$. Cette région autour de $\mathrm{E}_{0}$ est appelée "bottleneck region" des polaritons excitoniques sous forte intensité d'excitation. Elle joue un rôle important dans des processus de thermalisation et de collision entre quasi-particules. Pour $\gamma \neq 0$, en retournant à l'équation (19) et en négligeant la dispersion spatiale, nous remarquons que $\varepsilon(k, \omega)$ est complexe et possède une partie réelle $\left(\varepsilon_{1}\right)$ et imaginaire $\left(\varepsilon_{2}\right)$ :

$$
\begin{aligned}
\varepsilon(\omega) & =\varepsilon_{b}\left(1+\frac{\beta \omega_{0}^{2}\left(\omega_{0}^{2}-\omega^{2}\right)}{\left(\omega_{0}^{2}-\omega^{2}\right)^{2}+\omega^{2} \gamma^{2}}+i \frac{\beta \omega \gamma \omega_{0}^{2}}{\left(\omega_{0}^{2}-\omega^{2}\right)^{2}+\omega^{2} \gamma^{2}}\right) \\
& =\varepsilon_{1}(\omega)+i \varepsilon_{2}(\omega)
\end{aligned}
$$

$\varepsilon_{1}$ et $\varepsilon_{2}$ ont des formes Lorentziennes, et, pour $\gamma \rightarrow 0, \varepsilon_{2}(\omega)$ tend vers une fonction $\delta\left(\omega-\omega_{0}\right)$ et $\varepsilon_{1}(\omega)$ montre une singularité à $\omega=\omega_{0}$ Si l'on introduit l'indice de réfraction complexe $\tilde{n}$ par:

$$
\tilde{n}(\omega)=\sqrt{\varepsilon(\omega)}=n(\omega)+i \xi(\omega)
$$


on obtient :

$$
\begin{aligned}
& \varepsilon_{1}(\omega)=n^{2}(\omega)-\xi^{2}(\omega) \\
& \varepsilon_{2}(\omega)=2 n(\omega) \xi(\omega) \\
& \text { ou encore : } \\
& n(\omega)=\left[\frac{1}{2}\left(\varepsilon_{1}+\left(\varepsilon_{1}^{2}+\varepsilon_{2}^{2}\right)^{1 / 2}\right)\right]^{1 / 2} \\
& \xi(\omega)=\left[\frac{1}{2}\left(-\varepsilon_{1}+\left(\varepsilon_{1}^{2}+\varepsilon_{2}^{2}\right)^{1 / 2}\right)\right]^{1 / 2}
\end{aligned}
$$

Avec ces dernières définitions, la partie réelle de la quantité de mouvement devient :

$$
\operatorname{Re}(k)=\omega n(\omega) / c
$$

qui gouverne la propagation des polaritons, et le coefficient d'absorption $\alpha$ est donné par

$$
\alpha=2 \omega \xi(\omega) / c
$$

Le facteur 2 dans l'équation (27) a son origine dans le fait que le coefficient d'extinction $\xi$ décrit la diminution de l'amplitude d'un champ électromagnétique se propageant dans le milieu quand $\alpha$ est celui de l'intensité. Cette dernière est proportionnelle à l'amplitude du champ au carré.

À partir de l'équation (22) nous pouvons déterminer les propriétés de propagation et d'atténuation d'un champ électromagnétique dans un matériau. À l'interface entre l'air et le matériau, il y a réflexion. $\grave{A}$ incidence normale, la réflexion $R_{\perp}$ est donnée par $n$ et $\xi$ et on obtient :

$$
R_{\perp}=\frac{I_{r}}{I_{i}}=\frac{[n(\omega)-1]^{2}+\xi(\omega)^{2}}{(n+1)^{2}+\xi(\omega)^{2}}
$$

où $\mathrm{I}_{\mathrm{r}}$ et $\mathrm{I}_{\mathrm{i}}$ sont respectivement les intensités réfléchie et incidente. À des incidences obliques, $R$ est donné par les formules de Fresnel [3]. Il est important de noter que $\xi(\omega)$ prend des valeurs importantes à la résonance $\left(\omega_{0}<\omega<\omega_{\mathrm{L}}\right)$ lorsque $n(\omega)$ est lui aussi important en dehors de cette région.

Normalement, $n(\omega)$ est déterminé par des méthodes linéaires comme par la méthode du prisme ou à partir des franges d'interférence ou ellipsométrie, mais nous verrons par la suite qu'il existe aussi des méthodes optiques non linéaires puissantes qui permettent de le faire.

Une méthode originale qui utilise une détection non linéaire est la méthode de temps de vol. La figure 3a schématise un montage type : des impulsions courtes, accordables en fréquence, traversent l'échantillon. Avec une impulsion synchrone de référence on enregistre la corrélation croisée des deux impulsions. À cause de l'indice de réfraction du matériau, l'impulsion test est retardée par rapport au temps de parcours dans le vide et le retard est ainsi mesuré. En connaissant l'épaisseur de l'échantillon, on peut calculer la vitesse de groupe $v_{g}$ qui est donnée par $\mathrm{d} \omega / \mathrm{dk}$. La figure $3 \mathrm{~b}$ montre un exemple où la vitesse de groupe d'un film de $\mathrm{CuCl}$ a été mesurée par cette méthode et la dispersion reconstruite [4].

Jusqu'à présent, nous avons discuté la réponse linéaire d'un matériau soumis à un champ électromagnétique. Si le système est soumis à une forte intensité d'excitation résonante, on créé des quasi-particules électroniques réelles. À cause de la nature de Fermion des électrons, 


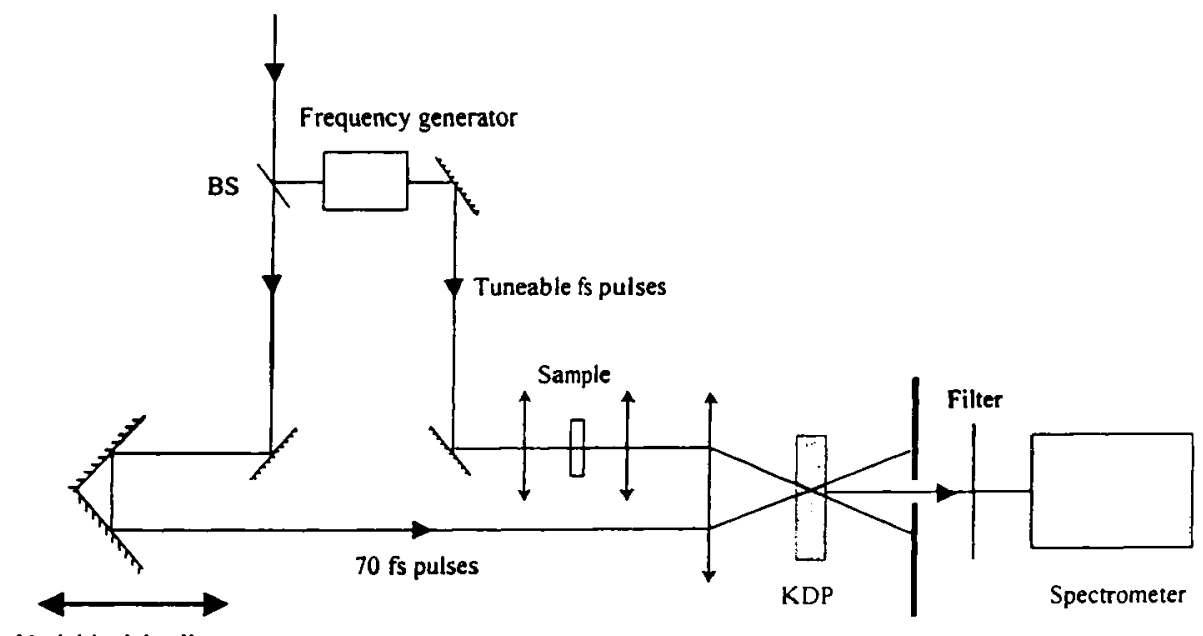

Variable delay line

Figure 3a : Expérience de temps de vol dans $\mathrm{CuCl}$ : montage.

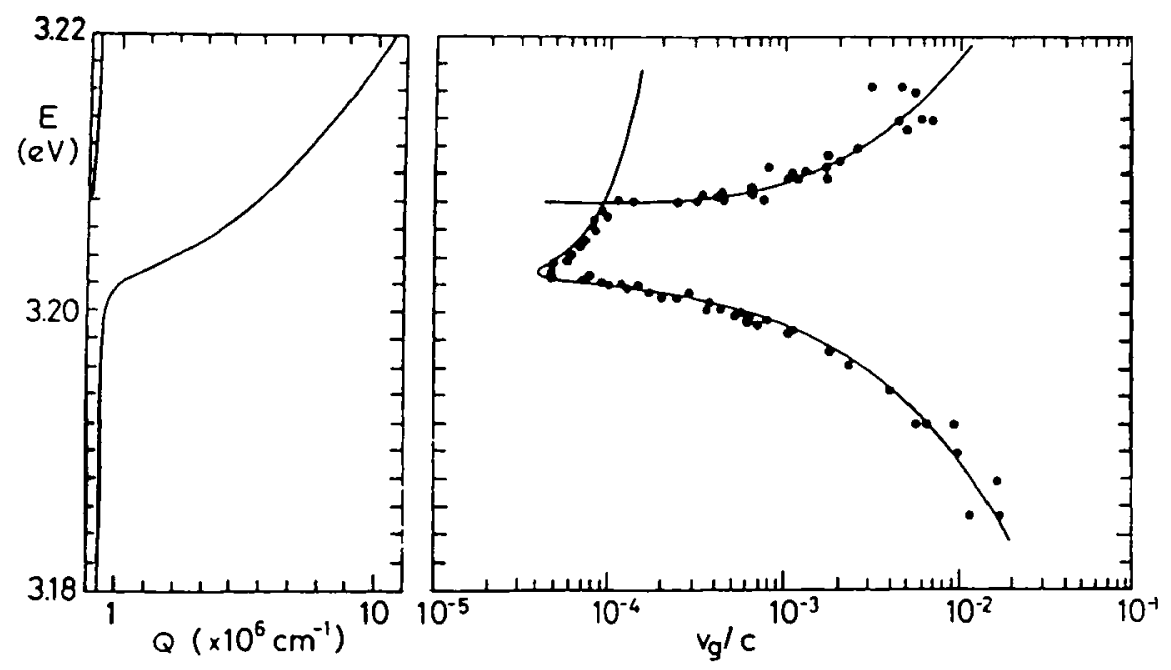

Figure 3b : Expérience de temps de vol dans $\mathrm{CuCl}$ : résultats expérimentaux (Réf. 4).

on ne peut pas exciter un deuxième électron dans le même état que celui qui est déjà excité. Ceci change donc, si l'on revient au modèle à un oscillateur (équation 19), la force des oscillateurs $\beta$ de la transition considérée et son damping $\gamma$. Ceci donne' lieu à des effets de saturation et à des processus de collisions. De plus, la forte intensité d'excitation peut changer par exemple l'écrantage des interactions entre les paires électron-trou et déplacer ainsi la fréquence propre $\omega_{0}$ de l'oscillateur. Elle est aussi directement modifiée par l'effet Stark optique. De plus, à cause de l'énergie déposée dans l'échantillon, la température $\mathbf{T}$ peut augmenter ce qui modifie $\gamma$ et $\varepsilon_{b}$. Comme nous le verrons, ces deux grandeurs sont aussi directement accessibles par des transitions à plusieurs photons. De plus (en considérant l'équation 17) comme nous le verrons au paragraphe 4 , le fait d'exciter en résonance un semiconducteur peut peupler ces niveaux, et leur excitation possède une certaine durée de vie. 
Ainsi, à partir de ces niveaux, de nouvelles transitions sont possibles, qui n'existent pas lorsqu'il n'y a que le niveau fondamental de peuplé.

Pour conclure ce paragraphe, il est important de noter que les propriétés optiques d'un matériau sont données par la fonction diélectrique $\varepsilon(k, \omega)$ qui, dans le modèle à un oscillateur, dépend de cinq quantités : $\varepsilon_{b}, \omega_{0}, M, \gamma$ et $\beta$. Sous forte excitation résonante, ces quantités sont modifiées et des nouvelles transitions apparaissent. L'ensemble donne lieu à des nonlinéarités optiques. Ces non-linéarités dépendent de la variation temporelle de l'impulsion excitatrice. De plus, puisque des quasi-particules réelles sont excitées, les systèmes montrent une dynamique propre. Ils apparaît donc des phénomènes optiques cohérents et incohérents qui peuvent être étudiés par de différentes méthodes de spectrocopie non linéaire. La compréhension des processus qui sont à leur origine est primordiale pour la conception des dispositifs optoélectroniques.

\section{SPECTROSCOPIE DANS L'ESPACE RÉCIPROQUE}

\subsection{Absorption à plusieurs photons non résonants}

La spectroscopie optique non linéaire est un outil puissant pour étudier les propriétés et la nature des états d'un matériau. La méthode la plus simple est l'étude de l'absorption à deux photons. Nous discutons cette méthode tout d'abord pour déterminer la dispersion de polariton excitonique d'un corps modèle : le chlorure de cuivre.

Comme indiqué dans le chapitre "Non-linéarités dans les matériaux semiconducteurs" pour discuter les transitions optiques (linéaires ou non linéaires), nous devons tout d'abord discuter les règles de sélection. $\mathrm{CuCl}$ est un semi-conducteur à large bande interdite $\left(E_{\mathfrak{g}} \sim 3,4 \mathrm{eV}\right.$ à température ambiante) et cristallise dans la structure de la blende (groupe ponctuel $T_{d}$ ). Sa bande de conduction est deux fois dégénérée et possède son minimum au point $\Gamma$ ayant la symétrie $\Gamma_{6}$. Les bandes de valence ont la symétrie (comme la plupart des semi-conducteurs de structure cubique) $\Gamma_{8}$ et $\Gamma_{7}$. La zone interdite est directe, située au point $\Gamma$. La figure 4 donne cette structure de bande $\mathrm{E}(\mathrm{k})$ autour du point $\Gamma$.

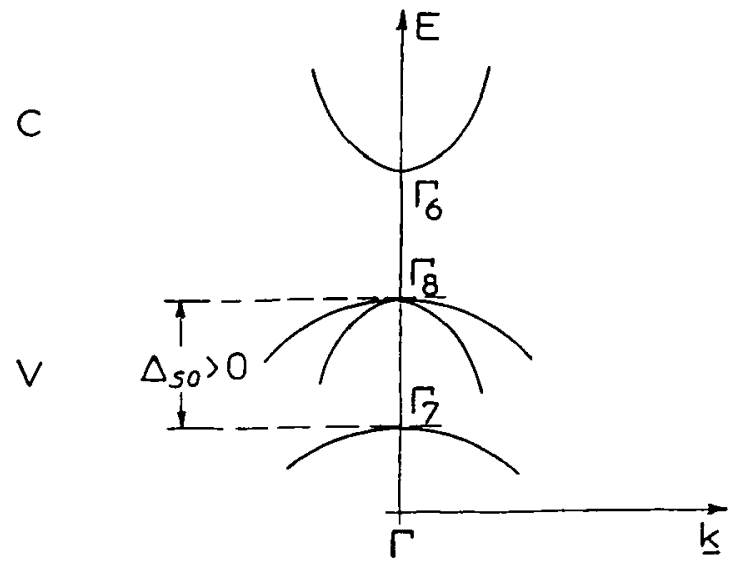

Figure 4 : Structure de bande typique des semi-conducteurs de structure de la blende. 
Les transitions

$$
\begin{gathered}
\Gamma_{8} \otimes \Gamma_{6}=\Gamma_{3} \oplus \Gamma_{4} \oplus \Gamma_{5}\left(\mathrm{Z}_{12}\right) \text { et } \\
\Gamma_{7} \otimes \Gamma_{6}=\Gamma_{2} \oplus \Gamma_{5}\left(\mathrm{Z}_{3}\right)
\end{gathered}
$$

entre les deux bandes de valence et la première bande de conduction font intervenir leur produit de représentations irréductibles. Ils sont permis, ce qui signifie que la transition est dipolactive (le dipole, vecteur de coordonnées $x, y, z$, se transforme comme $\Gamma_{5}$ en $T_{d}$ ). Par couplage spin-orbite, les deux bandes de valence sont séparées par $\Delta_{\text {So. }}$. La structure de bande donne donc lieu à deux séries d'excitons $[1,2]$ notées $Z_{12}$ et $Z_{3}$.

$\mathrm{CuCl}$ a la particularité que $\Delta_{\mathrm{SO}}=-81 \mathrm{meV}$ est négatif [1], donc la série excitonique $Z_{3}$ est la plus basse en énergie. Elle possède (équation. 29) dans l'état fondamental quatre états. L'un d'eux se transforme comme $\Gamma_{2}$ qui n'est pas optiquement actif et possède l'énergie la plus basse. $\mathrm{Si}$ " $\mathrm{z}$ " est la direction de propagation, par interaction d'échange analytique, deux états transverses (se transformant comme " $x$ " et " $y$ ") sont plus hauts en énergie que l'état de symétrie $\Gamma_{2}$. L'exciton longitudinal (se transformant comme " $\mathrm{z}$ ") est séparé d'eux par l'interaction d'échange non analytique [1]. Si l'on utilise de la lumière polarisée linéairement selon " $x$ " ou " $y$ ", ce champ ne se couple qu'avec un seul oscillateur donnant lieu à un polariton. Dans ce cas, les approximations faites pour obtenir l'équation (19) sont valables parce que les autres résonances se trouvent loin de l'état fondamental de la série $Z_{3}$.

Puisque la structure cristalline ne possède pas de centre d'inversion, les trois états de symétrie $\Gamma_{5}$ peuvent être excités par absorption à deux photons $[5,6]$. Le dispositif utilisé est décrit en référence [6]. Les impulsions d'un laser Nd:Yag d'une durée de 20 ns excite à $E_{1}=1,1648 \mathrm{eV}$ un échantillon dans un cryostat à 1,5 K. Son intensité est mesurée par une photodiode. L'échantillon est en même temps éclairé par un flash provenant d'une lampe à Xénon avec une énergie de photon $\mathrm{E}_{2}$ autour de $2,03 \mathrm{eV}$. Sa transmission par l'échantillon est mesurée en fonction de l'énergie de photon avant et simultanément à l'excitation. Le changement de la transmission induit par le laser est évalué. Ainsi, le coefficient d'absorption à deux photons peut être déterminé comme le montre la figure 5.

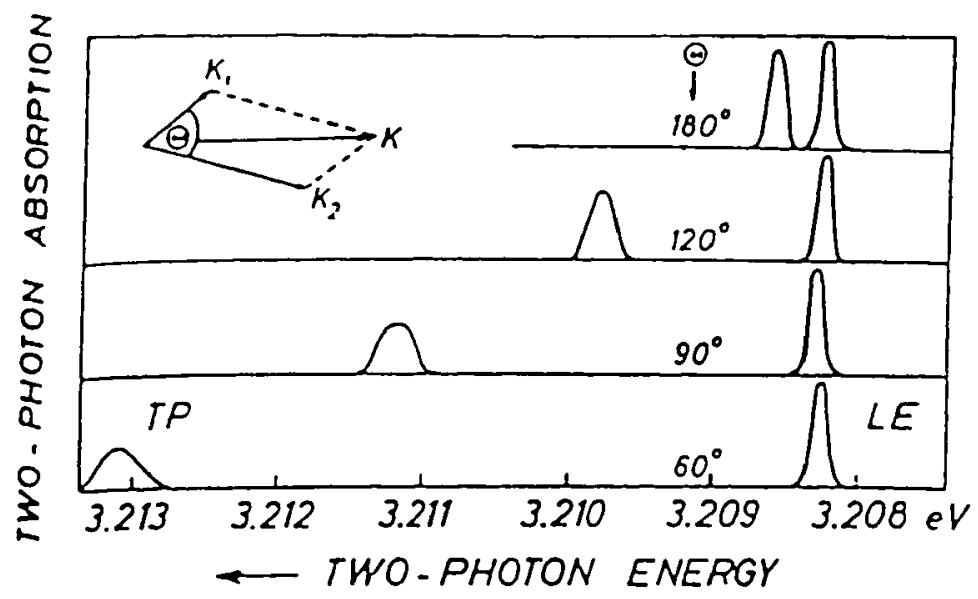

Figure 5a : Absorption à 2 photons dans $\mathrm{CuCl}[6]$. 


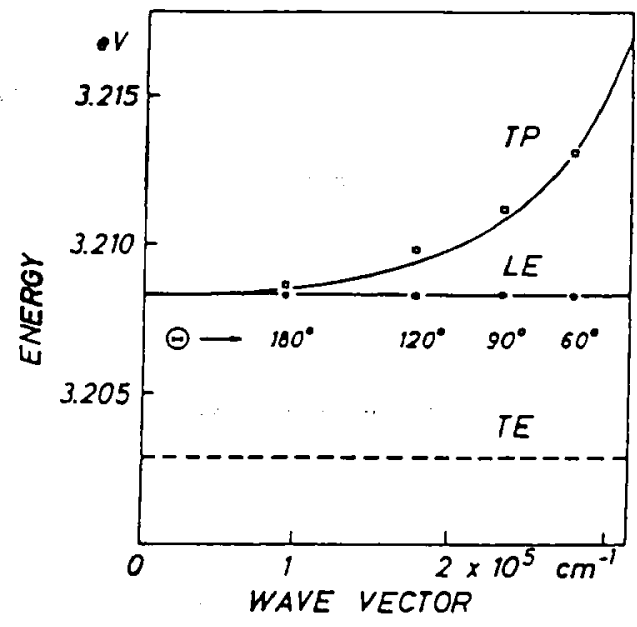

Figure 5b : Dispersion de polaritons dans $\mathrm{CuCl}[6]$.

Les deux photons d'énergies $\mathrm{E}_{1}$ et $\mathrm{E}_{2}$ et de vecteurs d'ondes $\vec{K}_{1}$ et $\vec{K}_{2}$ (dont la valeur et la direction dans le cristal sont bien connues d'après l'indice de réfraction et l'angle d'incidence sur la surface) créent la nouvelle quasi-particule en obéissant à la loi de conservation d'énergie et des moments. Ces quasi-particules sont créées avec le vecteur d'onde

$$
\vec{K}^{i}=\vec{K}_{1}+\vec{K}_{2}^{i}
$$

qui correspond à la condition d'accord de phase. De plus, ils ont une énergie de photon donnée par

$$
E^{i}=E_{1}+E_{2}^{i}
$$

où " $i$ " désigne les différents états finaux de la transition. Si l'on regarde les figures 1 et 2 , on $s$ 'aperçoit que selon la configuration géométrique,

$$
\left|K^{i}\right| \leq|K|+\left|K_{2}^{i}\right|
$$

En utilisant donc deux polaritons sur la branche inférieure, on peut atteindre la branche supérieure et la branche excitonique longitudinale si les règles de sélection le permettent. Comme constaté avant, ceci est le cas dans $\mathrm{CuCl}$ et on observe (figure 5a) deux raies d'absorption dont la position spectrale de l'une (b.s.p.) change si l'on change, l'angle $\theta$ entre les faisceaux. L'autre (correspondant à la création d'un exciton longitudinal) est spectralement fixe, c'est-à-dire que son énergie est indépendante du vecteur $\vec{K}^{i}$. Ceci est typique pour une branche qui ne montre qu'une faible dispersion spatiale parce que dans ces expériences $\left|\vec{K}^{i}\right| \leq 3.10^{5} \mathrm{~cm}^{-1}$.

À partir des équations (30) et (31), la dispersion des polaritons (linéaires) peut être reconstruite. Le résultat est montré sur la figure $5 \mathbf{b}$.

Les équations (30) à (32) impliquent que, par absorption à deux photons, la branche inférieure de polariton ayant une courbure concave ne peut pas être atteinte et, en conséquence, on ne peut pas déterminer la séparation $\hbar\left(\omega_{\mathbb{L}}-\omega_{0}\right)$ qui détermine la force 
d'oscillateur de la transition. Ceci devient possible, par contre [7], par une spectroscopie à 3 photons : à partir de 2 photons d'énergie et de moments différents, on excite (virtuellement) un troisième état qui possède le vecteur d'onde et la fréquence de différence des deux polaritons mélangés. Ce mode non propagatoire se couple avec un troisième polariton et ainsi on peut exciter un polariton sur la branche inférieure (b.i.p.) Ce processus est non résonant et relativement faible. Il est devenu possible parce que les lois de conservation d'énergie et du vecteur d'onde

$$
E_{f}=\sum_{i} E_{i} \text { et } \vec{K}_{f}=\sum_{i} \vec{K}_{i}
$$

ne font intervenir que des quasi-particules initiales et finales sans s'appliquer aux états intermédiaires dans les processus de génération.

Dans la référence [7], on utilise une cellule Raman de $\mathrm{H}_{2}$ à 40 bars. On génère des impulsions à l'énergie de photon de $\hbar \omega=0,64972 \mathrm{eV}$ à partir de l'émission d'un laser à $\mathrm{Nd}$ :Yag $(\hbar \omega=1,6475 \mathrm{eV})$. L'émission de ce laser, triplé en fréquence, pompe un laser à colorant, spectralement fin et accordable en fréquence. Sa transmission par l'échantillon est filtrée par un monochromateur et détectée par un photomultiplicateur. Comme précédemment, en changeant les angles entre les deux faisceaux et l'énergie de photon du laser à colorant, des quasi-particules sur les différentes branches peuvent être excitées. Elles ont des énergies et vecteurs d'ondes précises. Ceci donne lieu à une baisse d'intensité transmise du laser à colorant. Comme précédemment, en utilisant les équations (30) et (31), l'indice de réfraction du $\mathrm{CuCl}$ pour les différents photons ainsi que leurs angles d'incidence, on peut reconstruire la dispersion de polariton (Fig. 6). On ajuste après les paramètres de l'équation (19) ou même (dans le cas de plusieurs oscillateurs) de l'équation (17) et on détermine les valeurs des fréquences propres longitudinales ou transverses.

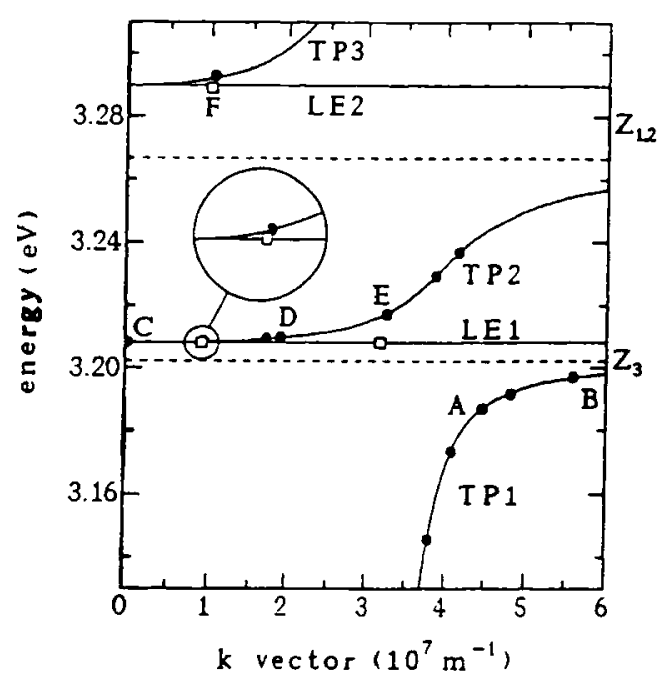

Figure 6 : Dispersion de polaritons dans $\mathrm{CuCl}[7]$.

Les méthodes d'absorption discutées demandent des échantillons épais de quelques millimètres. Hors résonance on peut estimer que le coefficient d'absorption à deux photons $\alpha_{2}$ 
est de l'ordre de $10^{-9} \mathrm{~cm} . \mathrm{W}^{-1}$. En conséquence, en utilisant des impulsions nanosecondes de puissance crête de quelques $\mathrm{MW} / \mathrm{cm}^{2}$, la variation de l'absorption induite par laser est faible. $\mathrm{Si}$ l'on veut étudier des films minces, on peut utiliser une autre technique plus sensible où on ne détecte pas la variation d'absorption mais on mesure l'émission des excitons créés auparavant par absorption à deux photons [5] . Cette technique possède un meilleur rapport signal/bruit. On ne peut, par contre, utiliser qu'une seule source excitatrice et perd ainsi les avantages que présentent une configuration avec deux sources de polarisation, de vecteur d'onde et d'énergie de photon variables indépendamment. Ceci est dû au fait que l'on ne peut pas distinguer si la luminescence observée était due à un état excité auparavant par deux photons provenant de la même source ou de deux sources différentes.

\subsection{Absorption et émission à plusieurs photons résonants : diffusion Hyper-Raman}

La figure 2 montre la dispersion des polaritons excitoniques et des excitons longitudinaux que nous voulons étudier dans la suite mais en utilisant des énergies de photons presque résonantes. Par rapport à la technique de spectroscopie à trois photons discutée auparavant, nous pouvons ainsi espérer augmenter la probabilité de transition de l'ordre d'un facteur $10^{6}$. Nous verrons, par contre, que l'utilisation des photons presque résonants peut également avoir des inconvénients.

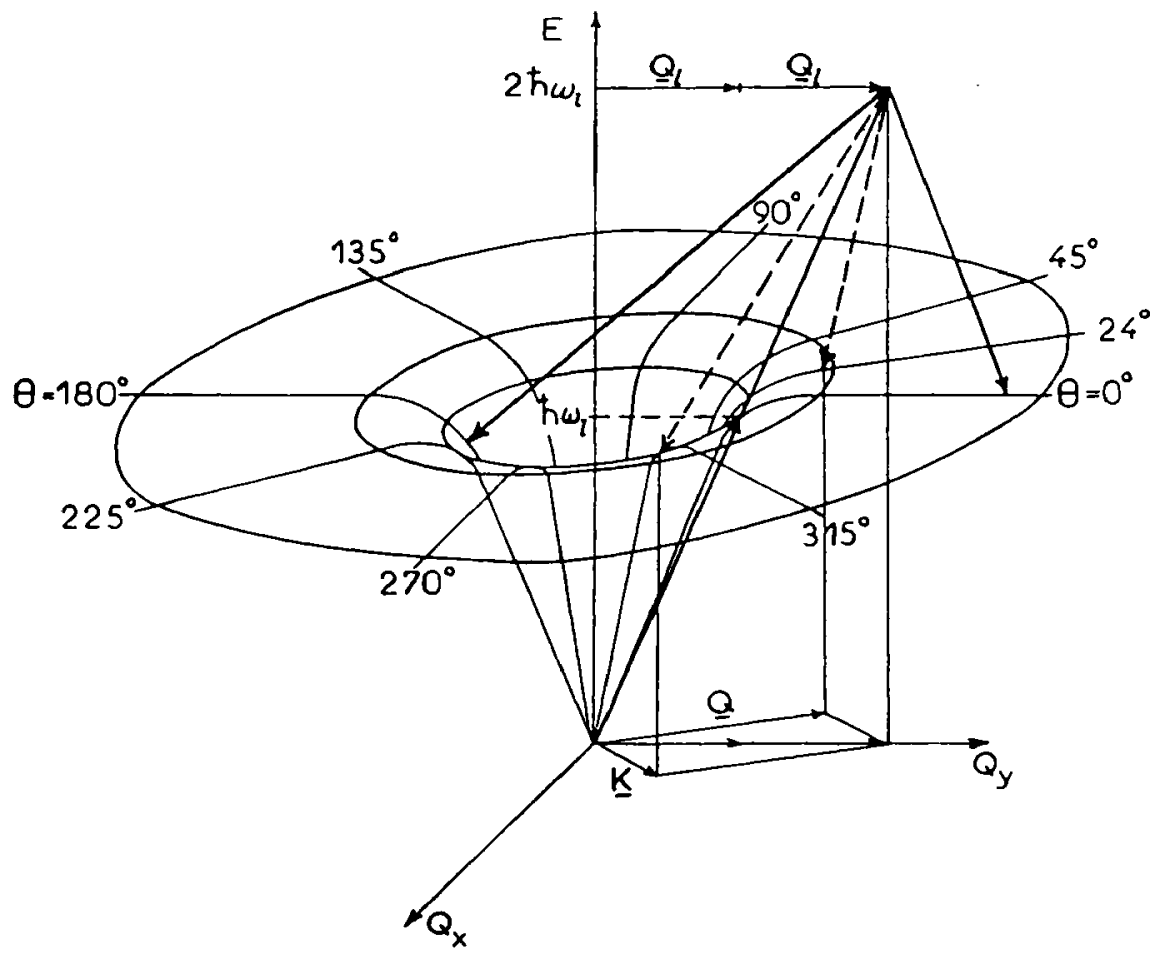

Figure 7 : Principe de la diffusion hyper-Raman excitonique.

Dans $\mathrm{CuCl}, 1$ 'approximation à un oscillateur est justifiée $[1,8-16]$ et la dispersion est 
isotrope. Nous la traçons (figure 7) dans le plan (X,Y). Nous excitons un échantillon avec un laser accordable d'énergie de photon $\hbar \omega_{1}$ et les polaritons ont le vecteur d'onde $\vec{Q}_{l} / / \vec{Y}$. On excite, par absorption à deux photons un état intermédiaire à l'énergie $2 \hbar \omega_{1}$ et de vecteur d'onde $2 \vec{Q}_{l}$. Sous conservation d'énergie et de moment (équations 30 et 31 ), cet état peut se décomposer spontanément en deux autres polaritons sur la b.i.p. (ou bien un exciton longitudinal et un b.i.p.). Discutons, comme il est indiqué dans la figure 7 , l'émission de l'échantillon en rétrodiffusion (flèches continues). On observe le processus où un b.i.p. avec un vecteur d'onde $\vec{Q}_{3} \approx-\vec{Q}_{1}$ est créé $(\theta=180)$ et étudié comme photon émis par l'échantillon. Le deuxième b.i.p. avec $\vec{Q}_{4} \approx 3 \vec{Q}_{l}(\theta=0)$ possède l'énergie $\mathrm{E}_{4} \approx \mathrm{E}_{0}$. D'après l'équation (31) l'énergie du polariton observé est donc $E_{3} \approx 2 \hbar \omega_{1}-E_{0}$, et la position spectrale varie donc avec $\hbar \omega_{1}$. Ceci est montré sur la figure 8 où une raie $\left(R_{T}\right)$ se déplace[10 - 12] si l'on change l'énergie de photon du laser excitateur $\hbar \omega_{1}$. La figure 9 montre la position spectrale des raies fines en fonction de $\hbar \omega_{\text {J. }}$ La pente de la raie $R_{\mathrm{T}}$ est égale à 2 .
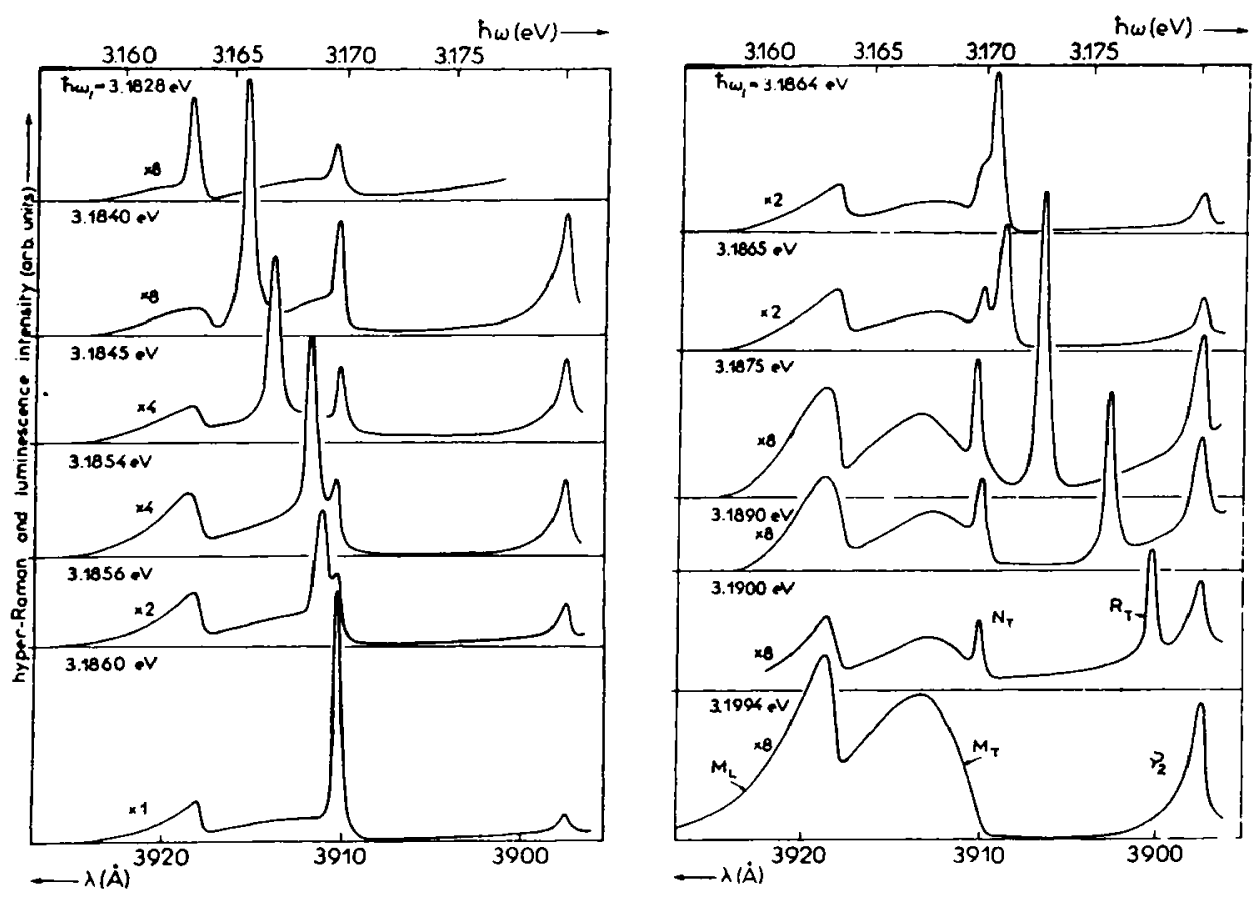

Figure 8 : Spectre d'émission du $\mathrm{CuCl}$ à $4 \mathrm{~K}$ pour différentes énergies de photon d'excitation.

Si l'on revient à la figure 7, on voit que le processus étudié n'est pas le seul : si l'on analyse (par exemple dans une configuration en transmission) l'émission de l'échantillon faisant un angle $\theta$ avec la direction d'excitation, on trouve trois raies d'émission. Une est proche de $E_{0}$ et dépend faiblement de $\hbar \omega_{1}$ et de $\theta$. La position spectrale des deux autres, notées $R_{T}^{+}$et $R_{T}^{-}$en dépendent fortement. La figure 7 (flèches pointillées) schématise la situation 
pour $R_{T}^{+}$, observé dans une direction de $24^{\circ}$ par rapport à $\vec{Q}_{l}$. La variation de la position spectrale avec $\theta$, pour $\hbar \omega_{1}$ donné, a son origine dans le fait que les deux polaritons finaux du processus de collision se trouvent dans la région de "bottleneck" de la branche de polariton où $\mathrm{E}$ varie fortement avec $|\mathrm{Q}|$.

Cette situation est montrée sur la figure 10 où on observe des raies d'émission $R_{T}^{+}$et $R_{T}^{-}$ à des positions spectrales différentes si l'on change l'angle d'incidence du laser $\alpha$ et garde la configuration $\left(\hbar \omega_{1}, \beta\right)$ constante. La figure 11 montre la position spectrale des raies pour des différentes configurations $\left(\alpha, \hbar \omega_{1}\right)$. L'indice de réfraction et les angles d'incidences $\alpha$ et $\beta$ déterminent l'angle de diffusion $\theta$ à l'intérieur de l'échantillon.
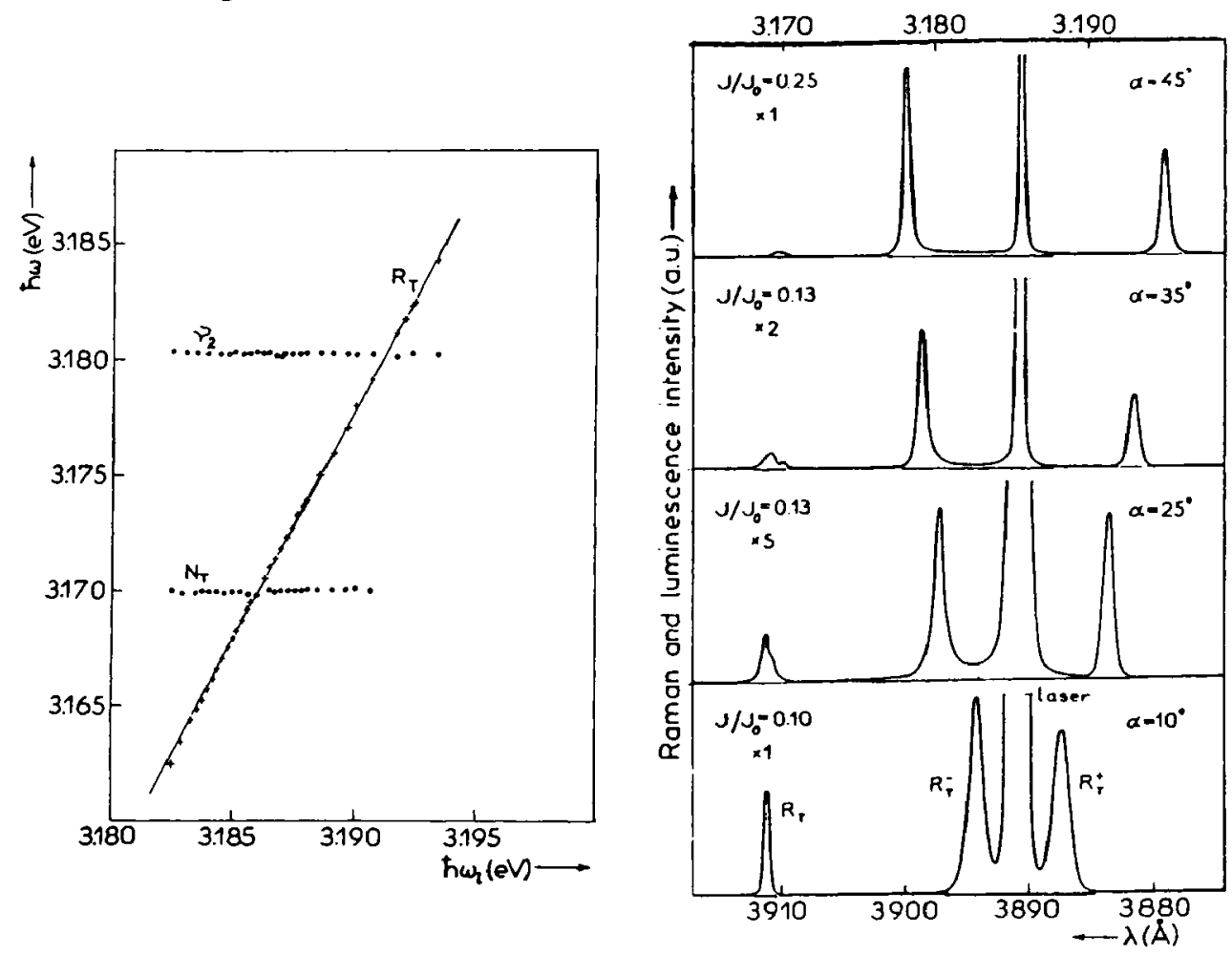

Figure 9 : Variation de la position des raies fines en fonction de $\hbar \omega_{1}$.

Figure 10: Spectre d'émission de $\mathrm{CuCl}$ pour $\hbar \omega_{1}=3,1856 \mathrm{eV}$ et différents angles d'incidence du laser $\alpha$ sur la surface de l'échantillon. L'angle que la direction de l'observation fait avec la surface de l'échantillon $\beta=0$ est fixe.

Si l'angle de diffusion $\theta$ est grand $\left(\theta-60^{\circ}\right)$ et si la polarisation de l'émission est dans le plan de diffusion, un des états finaux peut être un exciton longitudinal et une raie notée $R_{L}$ est observée. Elle a été mesurée pour deux configurations : rétrodiffusion (où le vecteur d'onde de l'exciton $\left.\mathrm{Q}_{\mathrm{ex}} \approx 3 \mathrm{Q}_{1}\right)$ et en transmission $\left(\mathrm{Q}_{\mathrm{ex}} \approx \mathrm{Q}_{1}\right)$. Un déplacement systématique de la raie $\mathrm{R}_{\mathrm{L}}$ est observé qui est attribué à la dispersion spatiale de l'exciton longitudinal [12]. 
Afin d'atteindre la b.s.p., on ne peut pas utiliser un seul laser d'excitation parce que le module du vecteur de $2 \bar{Q}_{l}$ est trop important pour que l'équation (33) possède une solution.

On peut, par contre, utiliser deux lasers à colorant différents et exciter l'échantillon des deux côtés [13]. Ainsi, le module du vecteur avec lequel l'état intermédiaire est excité est plus petit et un processus de recombinaison qui fait intervenir la b.s.p. est possible.

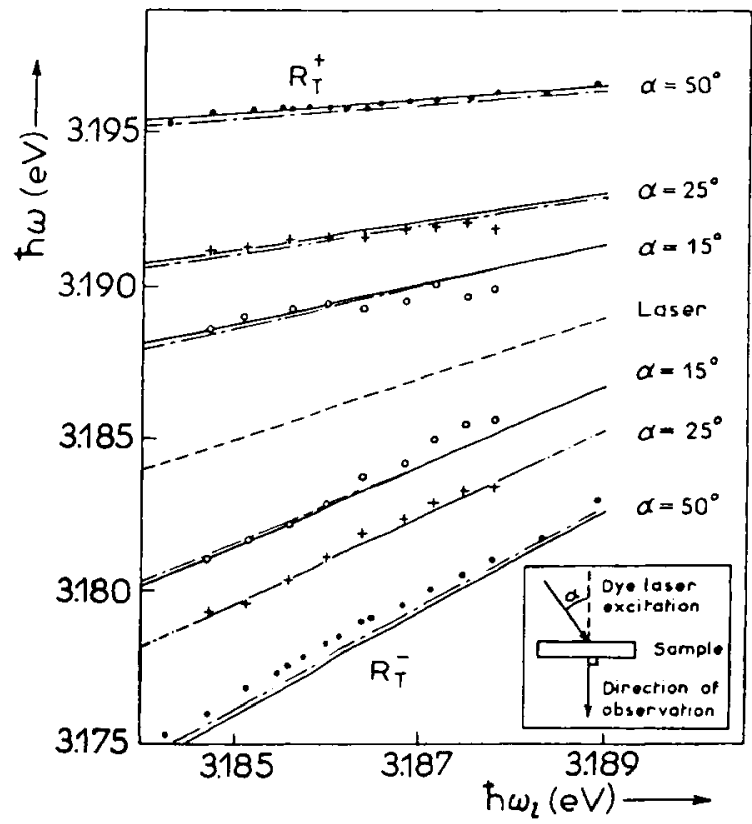

Figure 11 : Positions spectrales des raies $R_{T}^{+}$et $R_{T}^{-}$pour différents angles $\alpha$ en fonction de $\hbar \omega_{1}$.

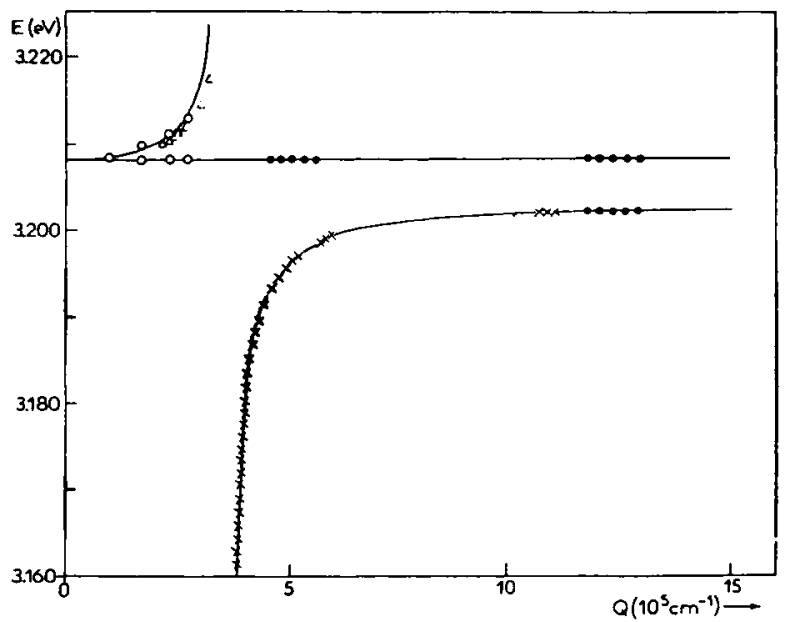

Figure 12 : Dispersion d'exciton et polariton obtenus par diffusion hyper-Raman $(\bullet, \times, \Delta)$ d'après les références [12,13] et absorption à 2 photons (o) d'après la référence [6]. 
L'analyse de l'ensemble des résultats obtenus par diffusion hyper-Raman est plus compliquée que dans le cas d'absorption à deux ou trois photons non résonants : comme le montre la figure 2 et l'équation (26), l'indice de réfraction $n$ varie fortement en fonction de l'énergie quand un polariton est dans la région du bottleneck. Pour $\gamma=0$, on fixe donc un jeu de paramètres qui définit la dispersion (équation. 17 ou 20). On mesure l'angle d'incidence du faisceau laser et son énergie de photon et on calcule $\vec{Q}$, à partir de l'équation (17). Par suite, on cherche la solution autoconsistante qui remplit les règles de conservation d'énergie et de moment de l'équation (33) avec la condition supplémentaire que la b.i.p. est émise par l'échantillon dans la direction $\beta$. On compare le résultat du calcul avec les mesures et ajuste éventuellement le jeu initial des paramètres. La solution que l'on obtient est donnée par les traits dans les figures (9) et (11). La figure 12 montre l'ensemble de ces résultats où on a attribué l'énergie des raies au module du vecteur d'onde calculé.

Afin de montrer la complexité de la situation dans d'autres semi-conducteurs dans la figure 13 on donne pour $\mathrm{CuBr}$ les positions spectrales des raies hyper-Raman en fonction de l'énergie de photon du laser dans une configuration de rétrodiffusion. La direction de propagation des polaritons est parallèle à [110], leur polarisation e // [001] ou e // [-110]. Ceci correspond aux représentations irréductibles $\Sigma_{1}$ et $\Sigma_{2}$ dans $T_{d}$ [17]. Comme je l'ai mentionné avant, dans la plupart des semi-conducteurs qui cristallisent dans la structure de la blende, $\Delta_{\text {so }}>0$ et la série excitonique la plus basse se forme à partir de la bande de valence de symétrie $\Gamma_{8}$ et de bande de conduction $\Gamma_{6}$. Ceci donne lieu à huit états excitoniques de symétrie $\Gamma_{3} \oplus \Gamma_{4} \oplus \Gamma_{5}$ dont la dégénérescence entre $\Gamma_{3} \oplus \Gamma_{4}$ et $\Gamma_{5}$ est levée par l'interaction

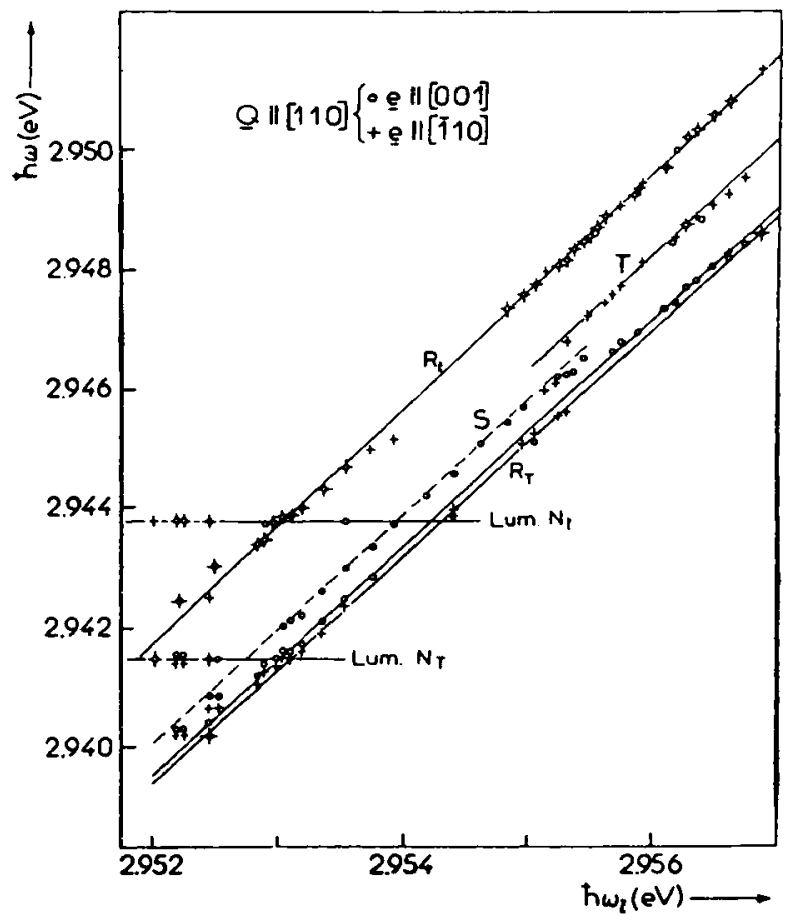

Figure 13 : Positions spectrales des raies hyper-Raman dans $\mathrm{CuBr}$ dans une configuration de retrodiffusion. 
d'échange analytique. $\grave{A} \vec{Q} \neq 0$, les états sont mélangés par des interactions qui dépendent de $\vec{Q}$ et peuvent devenir tous partiellement dipolactifs. Les règles de sélection ont été établies et les différentes transitions attribuées. Les mesures ont ainsi été effectuées pour différentes configurations [17] et les résultats ont été utilisés pour établir la dispersion de polariton de $\mathrm{CuBr}$ (figure 14).
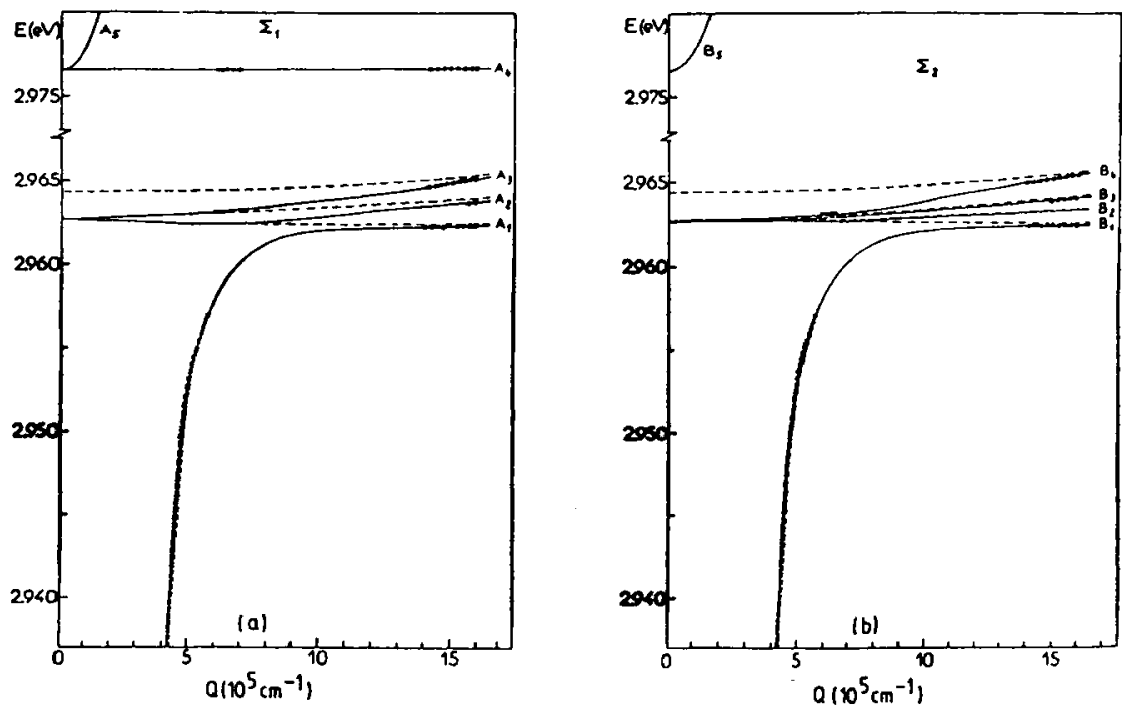

Figure 14 : Dispersion de polaritons de $\mathrm{CuBr}$ pour les représentations irréductibles $\Sigma_{1}$ et $\Sigma_{2}$.

\subsection{Biexcitons et absorption à deux photons}

Comme nous l'avons souligné précédemment, la présence de résonances augmente la probabilité de transition dans un processus à plusieurs photons. Dans le cas de la diffusion hyper-Raman dans $\mathrm{CuBr}$ et $\mathrm{CuCl}$ ce ne sont pas seulement des excitons dipolactifs qui jouent le rôle des états intermédiaires presque résonants, mais il existe d'autres états intermédiaires : les biexcitons. Deux excitons peuvent se coupler et donner lieu à un état lié : le biexciton. Son état fondamental possède la symétrie $\Gamma_{1}$, et peut être atteint par absorption de deux photons de même direction de polarisation linéaire $[1,8]$. On peut donc créer les biexcitons réels avec un photon d'un laser pompe (d'énergie de photon $\hbar \omega_{1}$ ) spectralement fin et accordable et d'un continuum qui ici est préparé par l'émission d'un colorant dans un solvant. Notre montage est montré sur la figure 15. Il est similaire à celui de Staginnus sauf que les deux cuves à colorant sont pompées optiquement par le même laser impulsionnel. La figure 16 montre un spectre de transmission de $\mathrm{CuCl}$ sans (en pointillé) et avec excitation par le laser (trait plein) dont la diffusion Rayleigh est aussi détectée par le spectrographe. On observe que le spectre est modifié par l'excitation et notamment deux raies d'absorption notées IA et TPA apparaissent. Le maximum de la TPA se déplace quand on change l'énergie de photon du laser $\hbar \omega_{1}$. Si on trace la position du dip TPA $\left(\hbar \omega_{c}\right)$ en fonction de $\hbar \omega_{1}$, on obtient une droite. La pente de la droite est de -1 , indiquant que la somme des deux énergies de photon est une constante :

$$
\hbar \omega_{\mathrm{c}}+\hbar \omega_{1}=6,372 \mathrm{eV}
$$




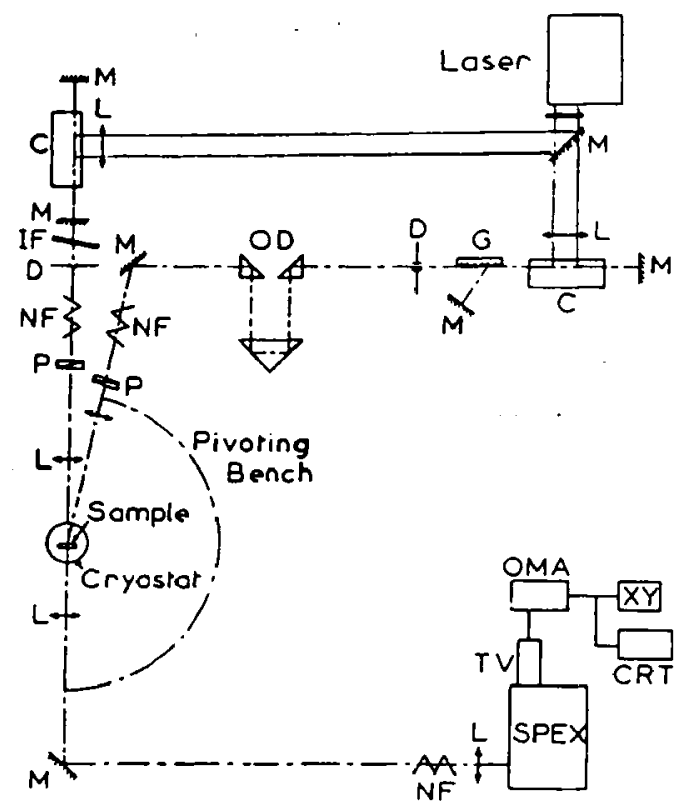

Figure 15 : Montage expérimental pour l'étude d'absorption à deux photons.

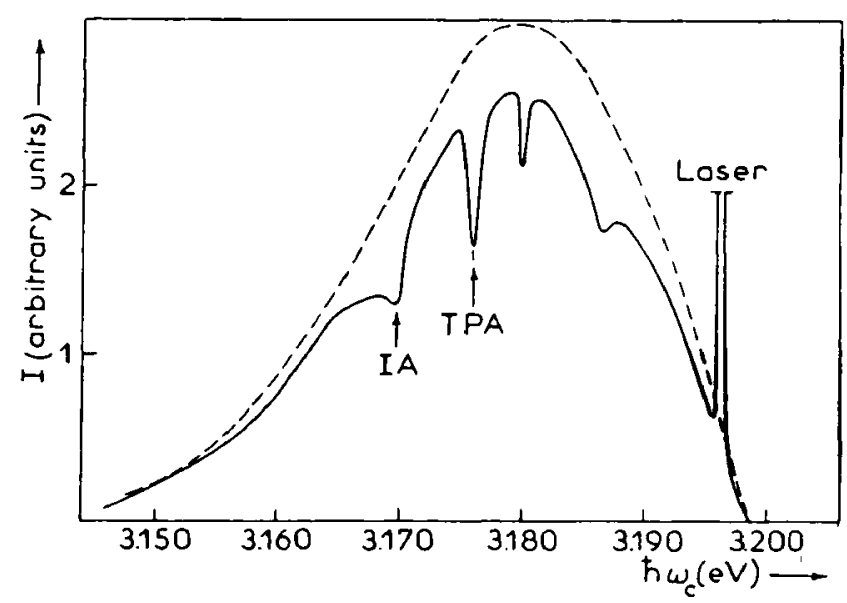

Figure 16 : Spectre de transmission de $\mathrm{CuCl}$ à $4 \mathrm{~K}$ sans (pointillé) et avec (trait plein) excitation par un laser.

Ceci prouve que l'état final est atteint par une absorption à deux photons simultanés. Si l'on change la polarisation linéaire d'un faisceau à l'autre, la'TPA' disparaît quand les polarisations des deux faişceaux sont croisées, indépendammentt de leur direction de propagation par rapport aux axes cristallins. De ce fait, on peut conclure que la symétrie de l'état final est de $\Gamma_{1}$, comme il était prédit théoriquement pour l'état fondamental du biexciton. De nouveau, on peut varier le vecteur d'onde d'un état final $\mathrm{K}$ par la configuration des deux faisceaux et par leur énergie de photon. Cette technique peut être utilisée pour déterminer la 
dispersion des biexcitons. Nous avons fait ces mesures dans $\mathrm{CuBr}$ qui possède six états de biexciton dont la dégénérescence est levée par l'interaction d'échange trou-trou. Ils ont la symétrie $\Gamma_{1}, \Gamma_{3}$ et $\Gamma_{5}$ à $\mathrm{K}=0$ et ont pu être identifiés en utilisant des sources polarisées linéairement [18]. La figure 17 montre le spectre de transmission [19] de $\mathrm{CuBr}$ dans une configuration parallèle et antiparallèle et la figure 18 la dispersion [20] avec le résultat d'un calcul pour des branches optiquement actives ou non. Dans ces figures, les vecteurs d'ondes sont calculés à partir des dispersions de polariton connues par diffusion hyper-Raman et les angles d'incidence des faisceaux, et les points donnent les énergies mesurées.

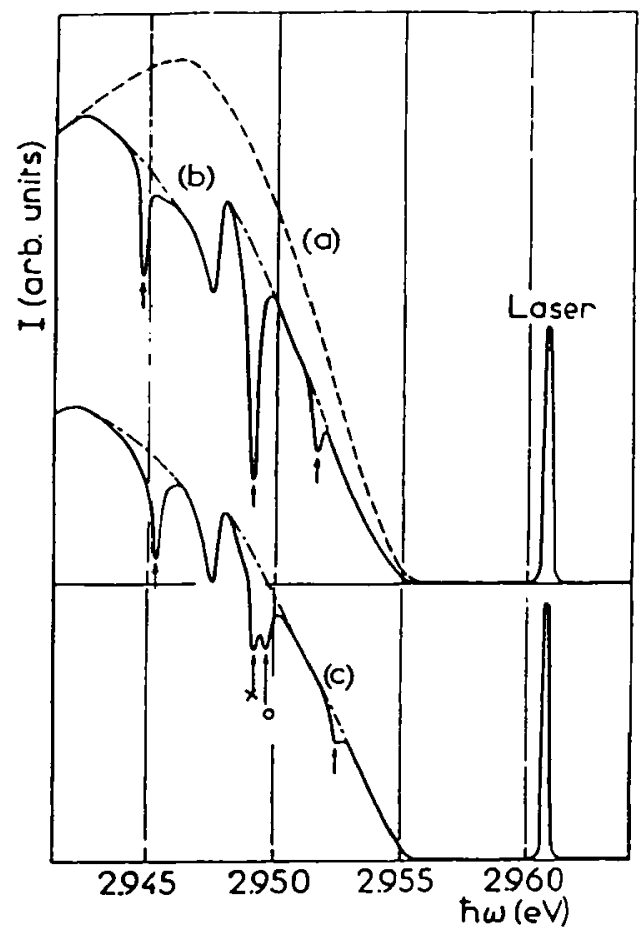

Figure 17 : " TPA " dans CuBr a/sans excitation b/continuum et laser antiparallèle - c/parallèle.

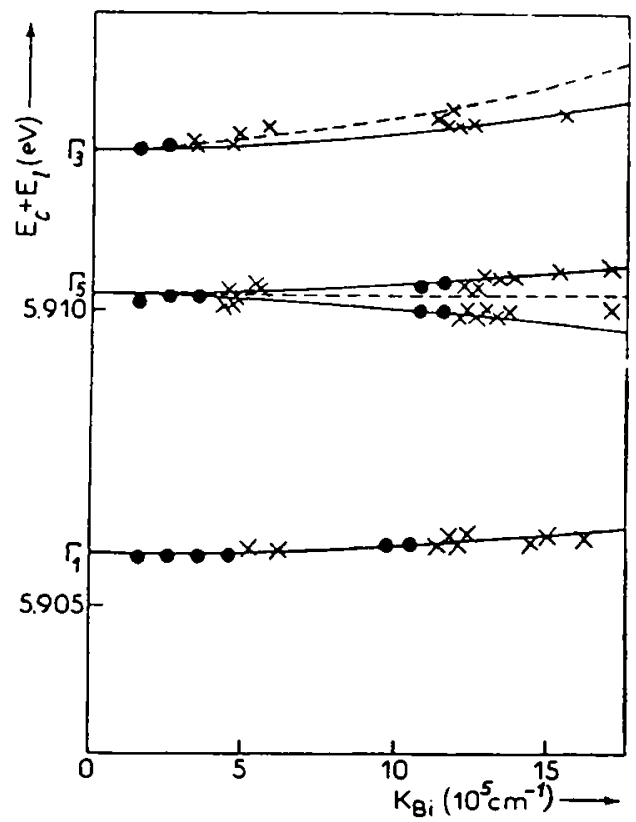

Figure 18 : Dispersion des biexcitons dans $\mathrm{CuBr}$. Traits pleins : niveaux accessibles par absorption à deux photons, pointillés : niveaux inaccessibles.

\subsection{Diffusion Hyper-Raman à forte intensité d'excitation.}

Revenons à $\mathrm{CuCl}$. Après avoir identifié le niveau biexcitonique il est clair que, si un tel niveau existe, il aura des effets non linéaires, c'est-à-dire qu'il modifiera la dispersion de polariton et l'absorption sous forte intensité d'excitation. À ce propos, il faut distinguer deux cas : le cas dégénéré (un seul faisceau modifie la fonction diélectrique à sa propre fréquence) et le cas non dégénéré (il modifie la fonction diélectrique aussi à d'autres fréquences et peut ainsi modifier les propriétés de propagation et d'absorption d'un faisceau test). En utilisant la technique de la matrice densité [21 - 24], nous avons calculé la dispersion et l'absorption d'un faisceau près 
de la résonance excitonique (figure 19). À fortes intensités d'excitation, la résonance se déplace par effet Stark optique. De plus, la figure 20 donne la dispersion de polariton quand un faisceau pompe excite $\mathrm{CuCl}$ à l'énergie de photon $\hbar \omega_{\mathrm{p}}$. Une forte anomalie apparaît à l'énergie de photon $E_{B i}-\hbar \omega_{p}$ et de plus, la dispersion est changée dans une grande plage. En prenant les deux dispersions et en suivant la même procédure que précédemment pour le calcul des positions spectrales des raies hyper-Raman, on obtient le résultat donné sur la figure 21 par le trait plein. Il apparaît clairement que la dispersion est fortement déformée par la grande intensité d'excitation. Les anomalies apparaissent quand, soit le polariton observé sur la b.i.p. ou son complément se trouve dans la région de forte variation de $E_{1}$ avec $Q_{1}[25-$ 29]. Le fait que l'on observe les variations de la position de $R_{T}^{+}$et $R_{T}^{-}$avec l'intensité aussi quand le complément du polariton qui est observé se trouve dans la région perturbée, montre bien qu'il s'agit d'un effet dispersif et pas d'une simple réabsorption.

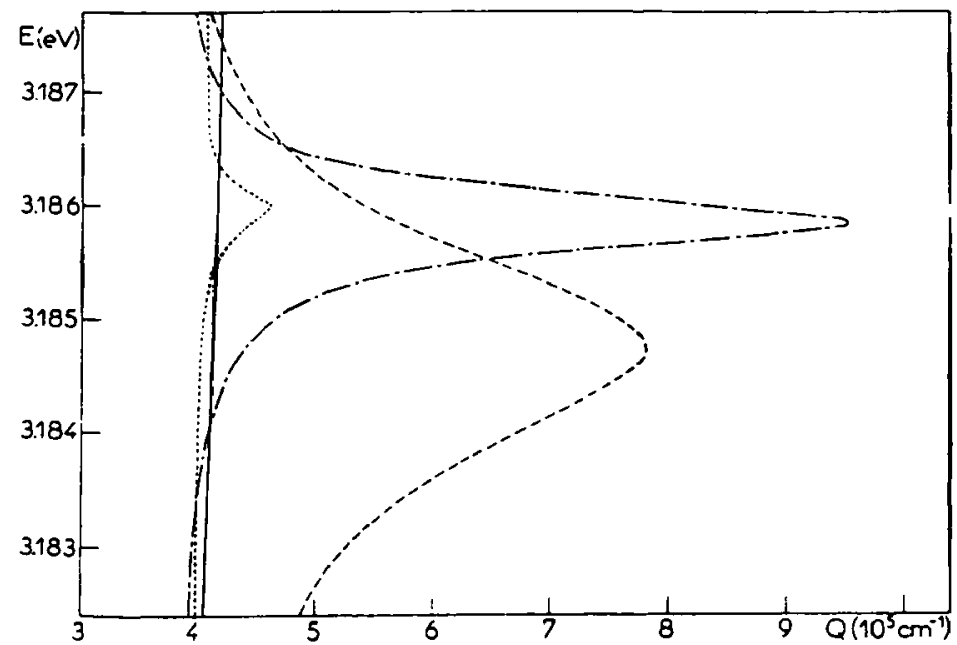

Figure 19a : Dispersion $\mathrm{CuCl}$ proche de l'énergie $E_{\mathrm{bj}} / 2$ pour différentes intensités d'excitation.

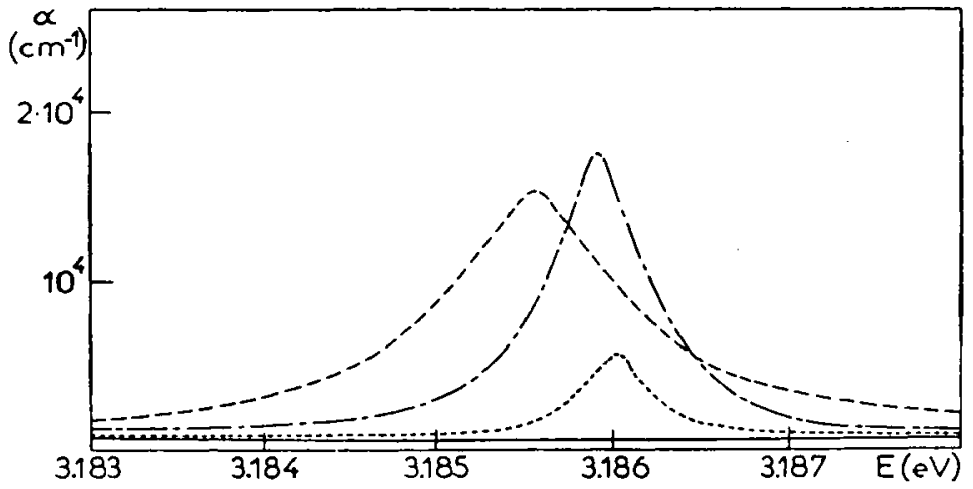

Figure 19b : Absorption de $\mathrm{CuCl}$ proche de l'énergie $\mathrm{E}_{\mathrm{b}} / 2$ pour différentes intensités d'excitation. 


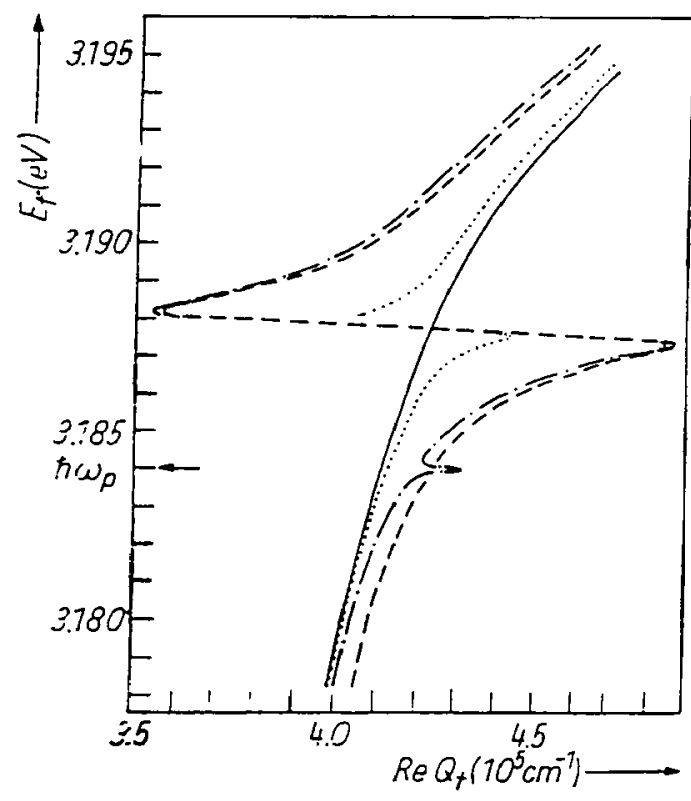

Figure 20 : Dispersion d'un faisceau test au voisinage de $E_{b i} / 2$ quand un faisceau pompe excite $\mathrm{CuCl}$ à

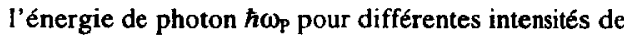
pompe.

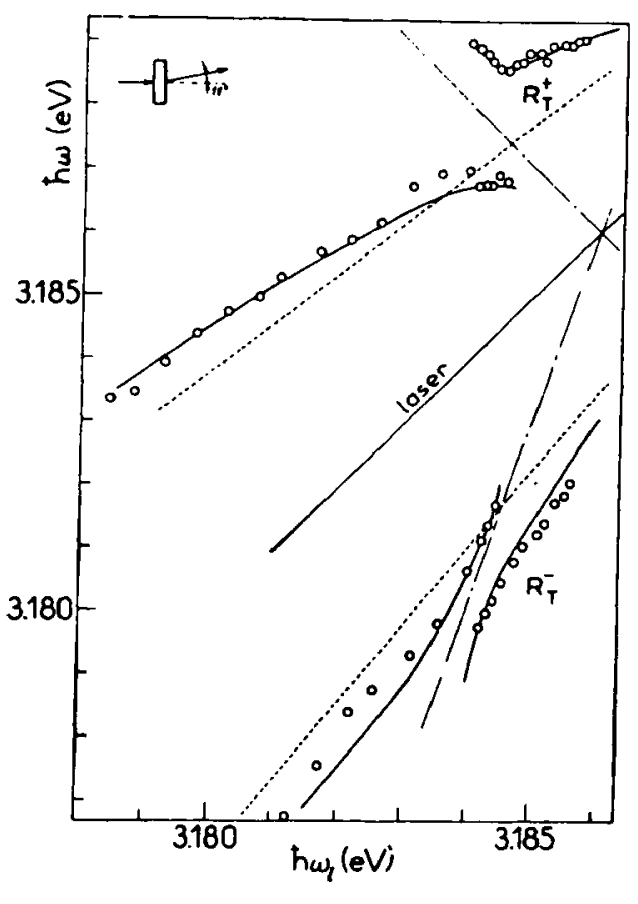

Figure 21 : Positions de Raies hyper-Raman à faible intensité d'excitation (en pointillé) et forte intensité d'excitation (cercles). Le trait plein donne les positions calculées en tenant compte de la renormalisation de la dispersion de polariton.

\subsection{Absorption induite dans $\mathrm{CuCl}$}

Revenons à la figure 16 de $\mathrm{CuCl}$ et la raie notée $\mathrm{AI}$. À partir de notre connaissance des énergies du biexciton, des excitons et de leurs masses effectives, nous pouvons l'identifier comme étant due à une transition entre des polaritons thermalisés sur la b.i.p. et le biexciton. Parce que la masse effective du biexciton est à peu près le double de celle de l'exciton, on obtient des raies spectralement larges si les excitons sont distribués dans leurs bandes [30]. Cette distribution d'excitons peut être décrite par une température effective. Nous avons étudié l'évolution de cette distribution en temps en excitant $\mathrm{CuCl}$ avec la quatrième harmonique d'un laser Nd: Yag à modes couples. Les impulsions ont une durée de $30 \mathrm{ps.} \mathrm{Une}$ partie du faisceau est focalisée dans une cuvette, contenant du $\mathrm{D}_{2} \mathrm{O}$. Par effet de Raman stimulé, un continuum, spectralement large et de même durée que les impulsions laser est créé et nous sert comme impulsion test. Après passage par une ligne de retard, le faisceau test est focalisé sur l'échantillon qui est refroidi à $4 \mathrm{~K}$. Le spectre transmis est analysé en calculant l'absorption induite à partir du rapport des intensités test transmises par l'échantillon sans $\mathrm{J}_{0}\left(\omega_{1}\right)$ et avec $\mathrm{J}(\omega)$ excitation laser. La figure 22 montre le coefficient d'absorption induit $\alpha_{\mathrm{J}} \propto \ln \left(\mathrm{J}_{0} / \mathrm{J}\right)$. 


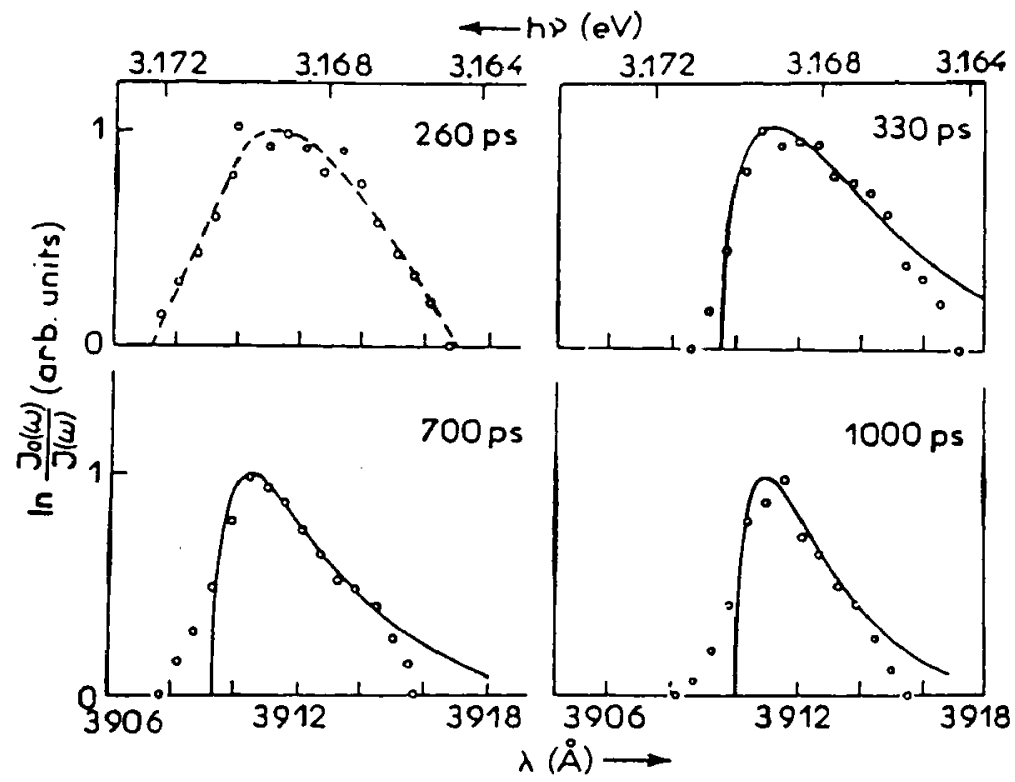

Figure 22 : Spectre d'absorption induite résolu en temps dans $\mathrm{CuCl}$.

On voit bien qu'à un retard $\tau$ de $260 \mathrm{ps}$ la distribution n'est pas entièrement thermalisée et pour $\tau>300$ ps on obtient une raie qui a la forme qui correspond à une distribution de Boltzmann inversée. Pour calculer la forme de la raie (trait plein) nous avons pris une distribution de Boltzmann pour les excitons transverses carractérisée par une température $T_{e}$ différente de celle du réseau. Nous avons négligé l'effet polariton, ce qui explique les différences avec les points expérimentaux à haute énergie de photon. L'absorption induite n'apparaît -due à une émission stimulée- qu'après $250 \mathrm{ps}$ et on voit (figure 23) la thermalisation du gaz d'exciton.

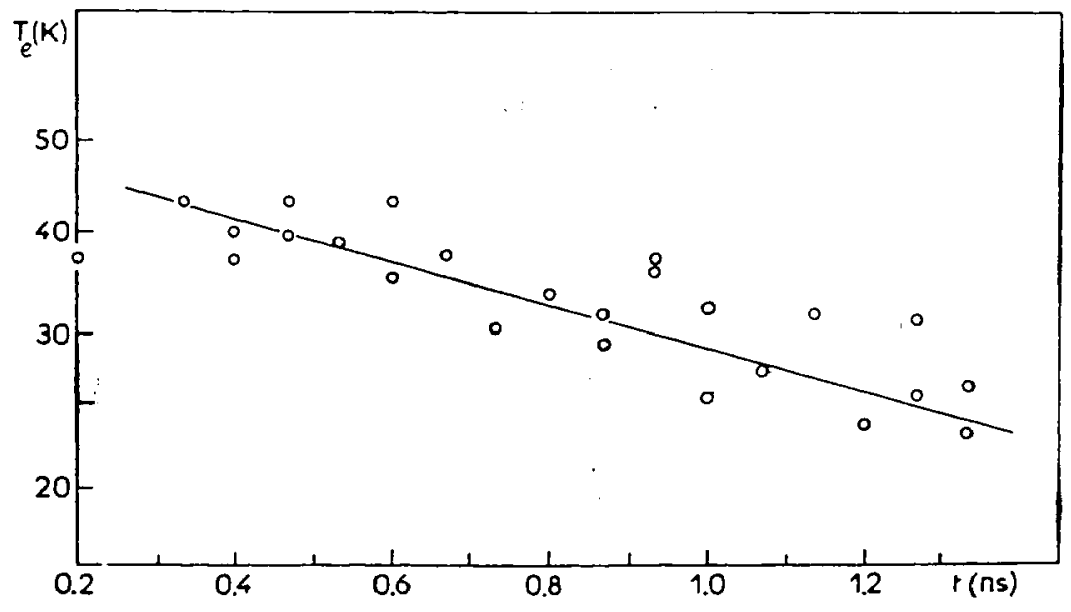

Figure 23 : Thermalisation des excitons dans $\mathrm{CuCl}$ a $4 \mathrm{~K}$. 


\section{ETUDE DE MODIFICATIONS D'ABSORPTION DANS DES SYSTÈMES DIFFÉRENTS}

\subsection{Limitation optique dans $\mathrm{C}_{60}$}

Nous avons jusqu'à présent étudié des systèmes invariants sous translation dans lesquels les transitions se font avec conservation d'énergie et des moments i. e. la dispersion de polaritons. Dans des systèmes où des molécules se trouvent dans un solvant ou des systèmes solides amorphes ou polycristallins, la règle de conservation des moments est relaxée et on étudie plutôt la variation de la fonction diélectrique macroscopique complexe $\varepsilon(\omega)$ qui est modifiée par l'excitation.

Dans le contexte discuté dans ce cours, la limitation optique est importante parce qu'elle trouvera des applications dans des problèmes de protection de dispositifs optiques. Les limitateurs optiques dont la transmission diminue en fonction de l'intensité lumineuse, peuvent être utilisés pour la protection de capteurs contre des grandes intensités lumineuses sans en réduire la sensibilité à faible intensité. La molécule de $\mathrm{C}_{60}$ est en principe un matériau prometteur pour une telle application car son absorption augmente fortement avec l'intensité lumineuse incidente. Plusieurs groupes ont étudié l'absorption non linéaire du $\mathrm{C}_{60}$ dissous dans des solvants ou déposé sous forme de couche mince. L'utilisation de matrices solides de Xérogel imprégnées de $\mathrm{C}_{60}$ présente néanmoins de nombreux avantages (excellente qualité optique, stabilité mécanique, seuil de dommage élevé et possibilité de choisir la concentration de dopage). Nous effectuons une étude des propriétés d'absorption non linéaire de tels verres de Xérogel massifs imprégnés de $\mathrm{C}_{60}$.

Afin de décrire l'absorption non linéaire de la molécule de $\mathrm{C}_{60}$, plusieurs auteurs proposent un modèle à cinq niveaux $[31,32]$. Chaque état électronique de la molécule est associé à un grand nombre d'états vibroniques. Le $\mathrm{C}_{60}$ possède des états excités de deux types, des états singulets $(S)$ et triplets $(T)$, correspondant à un spin total de 0 et 1 respectivement. Des porteurs dans le premier état excité $S_{1}$ sont transférés vers l'état $T_{1}$ de plus basse énergie que $S_{1}$, suivant un processus nommé "intersystem crossing", avec une constante de temps $\tau_{\text {sing-trip }}$ que nous déterminerons dans la section qui traite la dynamique. Le point clé pour l'existence d'une absorption qui augmente avec l'intensité incidente est le fait que la section efficace des états excités, $\sigma_{\mathrm{S} 1}$ ou $\sigma_{\mathrm{T} 1}$, respectivement pour l'état singulet $S_{1}$ ou l'état triplet $T_{1}$, soit plus grande que $\sigma_{\mathrm{SO}}$, la section efficace pour une absorption à partir de l'état fondamental $\left(S_{0}\right)$. Par conséquent, l'absorption totale de l'échantillon augmente avec la population des états excités, donc avec l'augmentation de l'intensité.

\subsubsection{Limitation optique}

Afin de caractériser les non-linéarités optiques de nos échantillons, nous avons étudié leur absorption en fonction de l'intensité d'excitation [33]. Nous avons mesuré la transmission d'un faisceau laser à travers l'échantillon pour différentes intensités lumineuses. La source laser utilisée est un laser $\mathrm{Nd}^{3+}$-YAG impulsionnel ( $25 \mathrm{ps}$ ) doublé en fréquence. Les mesures sont donc effectuées à température ambiante à une longueur d'onde de $532 \mathrm{~nm}$. Nous présentons ici les résultats obtenus avec un échantillon d'une épaisseur de $500 \mu \mathrm{m}$ dont la transmission linéaire est de $30 \%$ à $532 \mathrm{~nm}$. 
Nous mesurons la transmission du faisceau à travers l'échantillon en fonction de l'intensité lumineuse incidente. Une faible fraction du faisceau incident sert de référence afin d'éviter les erreurs de mesures dues aux fluctuations du laser. Les intensités incidentes et transmises sont déterminées à l'aide d'un même analyseur multicanal optique de type réticon. Le rapport des deux quantités nous donne la transmission de l'échantillon. Nous changeons la densité d'énergie sur l'échantillon grâce à des filtres atténuateurs. Nous les déplaçons de devant à derrière l'échantillon. De cette façon, une variation d'intensité mesurée avec la caméra réticon est uniquement due à un changement de transmission de l'échantillon. La figure 24 montre la transmission en fonction de l'énergie d'une impulsion.

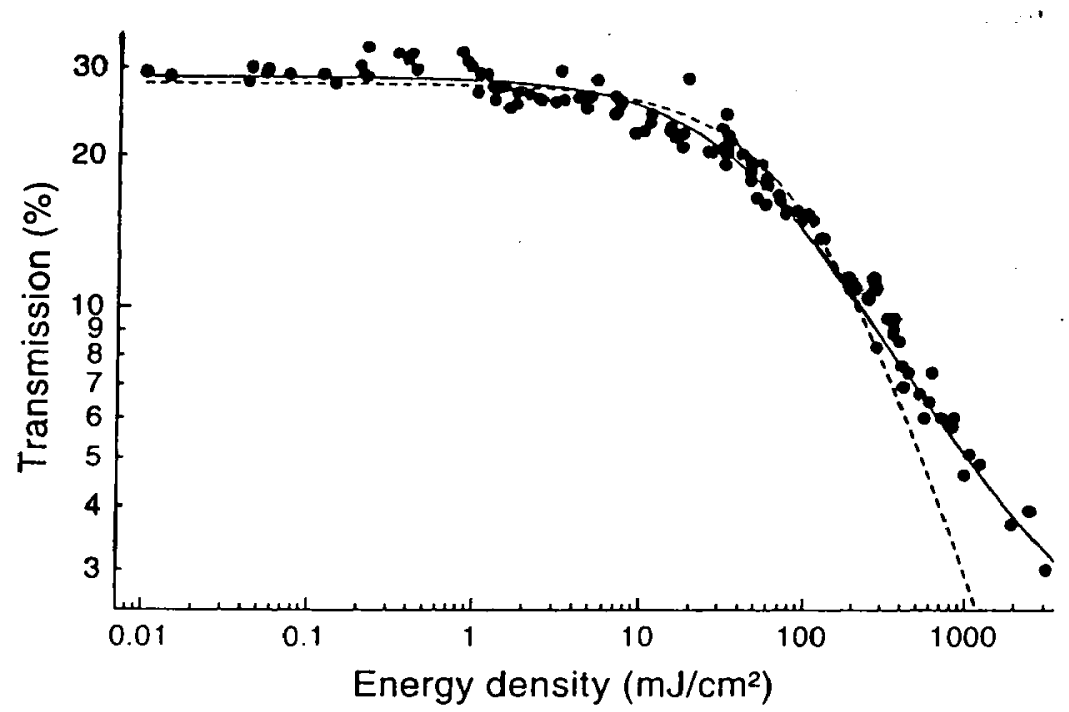

Figure 24 : Limitation optique de $\mathrm{C}_{60}$ : points : transmission mesurée, ligne hachée : absorption à deux photons successifs, trait plein : absorption à deux photons avec saturation.

Nous avons déplacé l'échantillon après chaque tir afin d'être sûr de ne pas avoir détérioré la surface de l'échantillon par une excitation préalable. En augmentant l'énergie des impulsions laser, une absorption induite apparaît. La transmission reste constante jusqu'à une valeur seuil à partir de laquelle elle diminue rapidement jusqu'à $10-20 \%$ de sa valeur pour des faibles intensités. La densité d'énergie maximale qui est de quelques $\mathrm{J} / \mathrm{cm}^{2}$ par impulsion laser, est supérieure au seuil de dommage du Xérogel. En conclusion, on peut constater que les Xérogels dopés au $\mathrm{C}_{60}$ montrent une limitation optique extraordinaire. la forme

Afin d'analyser nos résultats, nous avons supposé que l'absorption non linéaire possède

$$
\alpha=\alpha_{0}+\alpha_{2} I
$$

En intégrant l'équation différentielle

$$
\frac{\partial I(z)}{\partial z}=-\alpha I(z)
$$

pour $\alpha_{0}$ et $\alpha_{2}$ constant, nous obtenons la courbe en pointillé sur la figure 24. Afin d'améliorer l'accord entre points expérimentaux et le résultat de notre intégration à des fortes intensités, 
nous introduisons un terme de saturation $I_{\text {sat }}$ dans l'équation. (34). Dans ce cas, $\alpha_{2}$ prend la forme

$$
\alpha_{2}=\frac{\alpha_{2}^{0}}{1+I / I_{\text {sal }}}
$$

où $\alpha_{2}^{0}$ est une constante. La courbe en trait plein est calculée en utilisant $\alpha_{2}^{0}=11 \mathrm{~cm} / \mathrm{GW}$ et $\mathrm{I}_{\mathrm{sat}}=3 \mathrm{GW} / \mathrm{cm}^{2}$.

\subsubsection{Dynamique}

Afin de déterminer la dynamique du processus de limitation optique observé, nous avons effectué des expériences de pompe-sonde [33]. Cette méthode consiste à utiliser deux impulsions laser d'intensité différente comme le montre la figure 25 . Une première impulsion de forte intensité créé une importante population dans des états excités. Une deuxième impulsion d'intensité beaucoup plus faible sonde les changements de l'absorption engendrés par la première. En retardant l'impulsion sonde, on est ainsi capable de suivre l'évolution temporelle des populations excitées.

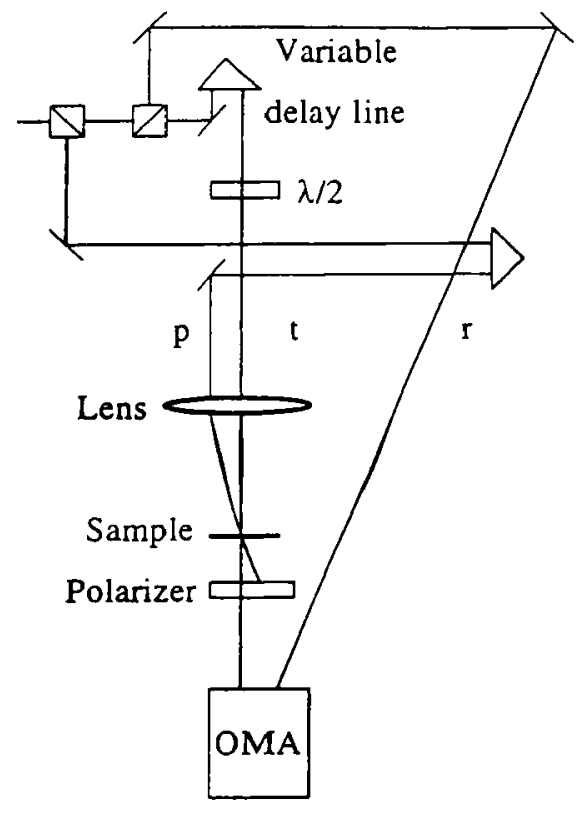

Figure 25 : Dispositif expérimental pour les expériences de transmission résolues en temps.

Une telle expérience a été effectuée en créant les faisceaux de sonde et de pompe à partir d'un même faisceau laser au moyen d'une lame séparatrice. Le laser utilisé est le même laser Nd:YAG décrit dans le paragraphe précédent. Une absorption induite est clairement visible. Nous en avons mesuré la dynamique en faisant varier le retard de l'impulsion sonde. Le résultat est donné par la figure 26. Nous interprétons la dynamique obtenue par une 
transition des excitons qui sont initialement créés dans les états singulets vers les états triplets. Nous en avons déterminé le rapport de deux des sections efficaces des états $S_{1}$ et $T_{1}$ ainsi qu'un $\tau_{\text {sing-trip }}$ de $150 \mathrm{ps}$, temps qui détermine la transition des porteurs de $S_{1}$ à $T_{1}$. Le résultat montre entre autre, que les états singulets sont à l'origine de l'absorption induite pour des

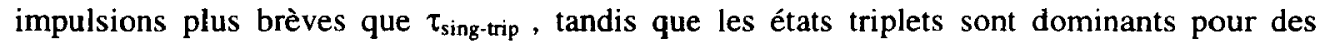
impulsions plus longues. Nous pouvons ainsi constater que les matrices sol-gel imprégnées au $\mathrm{C}_{60}$ jouent leur rôle de limiteur optique pour des impulsions de durée variable, mettant en jeu différentes populations excitées.

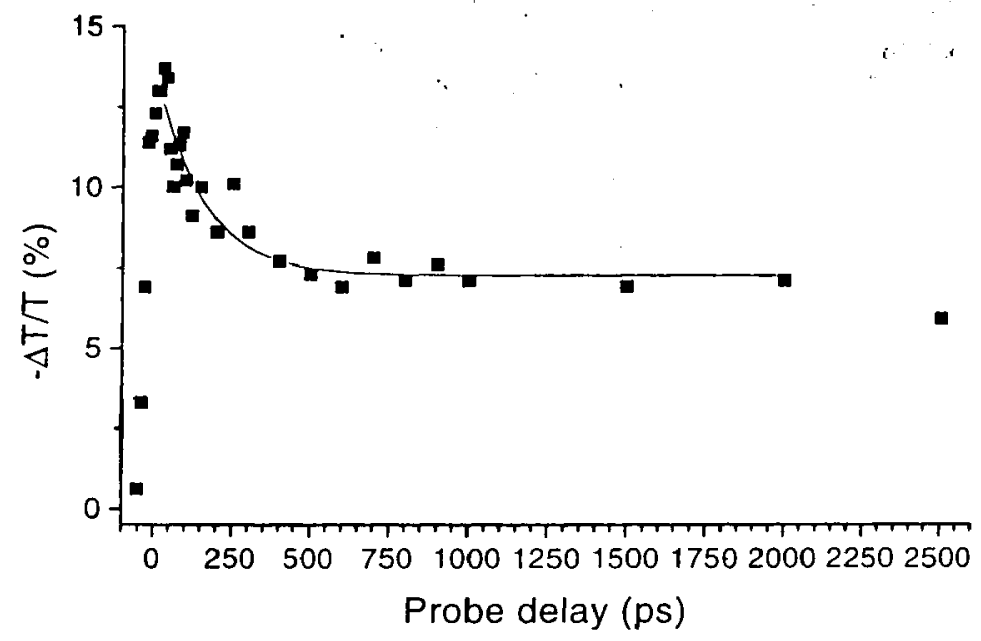

Figure 26 : Absorption induite résolue en temps.

Le même type d'expérience de pompe sonde a été largement appliqué à d'autres corps semi-conducteurs. Nous pouvons citer comme exemple nos travaux sur des films de CdZnTe sur substrat amorphe ou GaAs [34, 35], qui montrent une diminution de l'absorption à la place de l'absorption induite. Comme nous le verrons, ce comportement du au caractère polycristallin de l'échantillon est très différent de celui du monocristal massif.

\subsection{Déflection et absorption induites dans $\mathrm{Cd}_{1-\mathrm{x}} \mathrm{Zn}_{\mathbf{x}} \mathrm{Te}$}

Un exemple d'un système qui montre de fortes non-linéarités optiques absorptives et dispersives est le semi-conducteur ternaire $\mathrm{Cd}_{1-\mathrm{x}} \mathrm{Zn}_{\mathrm{x}} \mathrm{Te}$. Les composés II-VI que sont le tellurure de cadmium et de mercure ( $\mathrm{HgCdTe}$ ) et $\mathrm{CdTe}$ avaient été très étudiés pour des applications comme les détecteurs infrarouges. Ces détecteurs étaient à base de multicouches épitaxiées de $\mathrm{HgCdTe}$ déposées sur des substrats de CdTe. Or, les substrats doivent être de très bonne qualité pour éviter la diffusion de défauts du substrat dans la couche et une détérioration des performances du détecteur. Les cristaux de CdTe, bien qu'ils soient étudiés depuis très longtemps n'ont pas fourni le substrat de très bonne qualité que l'on escomptait. Les premières épitaxies réalisées sur de tels substrats ont révélé une importante densité de défauts. La qualité cristalline de la couche était altérée par les dislocations résultant des différences de maille pourtant faibles dans le cas de $\mathrm{HgCdTe} / \mathrm{CdTe}$. En outre, la croissance de 
CdTe était souvent très difficile à maîtriser et les défauts diffusaient dans la couche. L'adjonction de zinc au matériau binaire $\mathrm{CdTe}$ s'est avérée bénéfique pour la qualité du matériau obtenu.

L'idée d'introduire du zinc dans le tellurure de cadmium est essentiellement venue de l'instabilité latente de la liaison du composé binaire. La substitution des atomes de $\mathrm{Zn}$ aux atomes de $\mathrm{Cd}$ permet ainsi d'améliorer la structure du matériau. Ceci a fait de $\mathrm{Cd}_{1-\mathrm{x}} \mathrm{Zn}_{\mathrm{x}} \mathrm{Te}$ $(x \sim 0,04)$ un bon substrat ou une bonne couche intermédiaire entre le substrat et le détecteur lors de la croissance de ces couches. De plus, son paramètre de maille peut être ajusté avec celui de $\mathrm{HgCdTe}$ et son gap est suffisamment large pour éviter le confinement de porteurs minoritaires. On comprend mieux l'intérêt tout particulier porté à ce matériau $(x=0,04)$.

L'étude des non-linéarités optiques (indice de réfraction et absorption induite) est tout d'abord discutée. Nous avons vu précédemment que les spectres de transmission et d'absorption de $\mathrm{CdZnTe}$ ne présentent aucune résonance, nous observons seulement un bord d'absorption de forme exponentielle pour tous les échantillons quelle que soit leur concentration en zinc et en cadmium. Nous avons choisi de mesurer les changements non linéaires induits dans une région d'énergie inférieure à celle du bord d'absorption fondamental du cristal. Nous rappelons que le bord d'absorption dont nous parlons dans la suite est la région spectrale où le matériau commence à absorber le rayonnement et ne correspond pas au bord de la transition bande-à-bande fondamentale. Le faisceau test est choisi spectralement large (continuum), de façon à pouvoir sonder toute cette zone spectrale. Le faisceau pompe excite toujours en résonance et est accordable, sa longueur d'onde varie dans la même région du spectre visible.

\subsubsection{Etude de la variation d'absorption induite}

À température ambiante, nous faisons varier l'énergie des photons du faisceau pompe [36, 37] entre 2,07 et $2,16 \mathrm{eV}$. Les spectres de changement d'absorption obtenus sont représentés sur l'encadré de la figure 27 pour différentes énergies de photon du faisceau d'excitation.

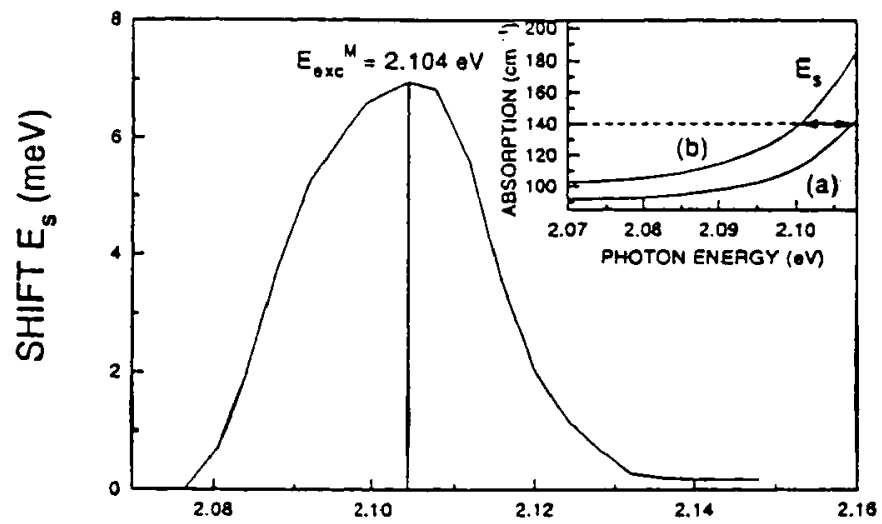

EXCITATION PHOTON ENERGY $E_{\text {exc }}(\mathrm{eV})$

Figure 27 : Déplacement du bord d'absorption par rapport au bord linéaire en fonction de l'énergie de photon d'excitation. 
À l'énergie de photon la plus faible $(2,077 \mathrm{eV})$, l'absorption du faisceau pompe correspond à la courbe (a). On constate qu'il n'y a pas de changement d'absorption. Au fur et à mesure que l'on augmente l'énergie des photons du faisceau pompe, une absorption de plus en plus grande du rayonnement incident apparaît à des énergies inférieures à la largeur de la bande interdite. Nous constatons un déplacement spectral du bord d'absorption vers les faibles énergies si l'énergie des photons de la pompe augmente. Lorsque cette énergie est de 2,104 eV (courbe (b)), l'effet de déplacement du bord d'absorption vers les faibles énergies (vers le rouge) est maximal. Si l'on augmente encore l'énergie de photon de la pompe, il y a un retour vers les hautes énergies jusqu'au bord du régime linéaire. Pour une même valeur du coefficient d'absorption, nous traçons la différence d'énergie $\Delta E$ entre les bords d'absorption de l'échantillon excité ou non, en fonction de l'énergie des photons d'excitation. La figure 27 montre ce spectre d'excitation. Nous faisons apparaître une bande centrée vers $2,104 \mathrm{eV}$ avec une largeur à mi-hauteur de $27 \mathrm{meV}$ (pour une concentration $\mathrm{x}=0,87$ ). Lors de la mesure du spectre d'excitation de la luminescence à la température ambiante et sous forte excitation lumineuse, nous avons constaté que la luminescence est maximale lorsque l'énergie de photon du faisceau d'excitation coöncide avec cette bande. Il y a donc une étroite relation entre la luminescence et l'absorption induite.

Pendant ces mesures, nous avons constaté que les non-linéarités optiques de CdZnTe sont très fortes (environ vingt fois plus grandes que dans le matériau binaire [38]) et donnent lieu à une forte focalisation ou défocalisation du faisceau test transmis par l'échantillon. En conséquence, pour mesurer ces changements et leur dynamique, nous avons ajouté au laser "test" des miroirs supplémentaires pour constituer une deuxième cavité et travaillé avec un laser accordable à contre-réaction distribuée. Les deux sources ont une durée d'impulsion de l'ordre de $150 \mathrm{ps}$ avec des fluctuations temporelles de l'ordre de $100 \mathrm{ps}$, ce qui donne lieu à une résolution globale d'environ $300 \mathrm{ps}$ du système. De plus, nous avons remplacé le spectrographe de la figure 15 par une simple caméra à réseaux de diodes (analyseur multicanaux optiques) qui mesure le profil spatial du faisceau transmis par l'échantillon avec ou sans excitation par laser. Grâce à des diaphragmes, la distribution spatiale de l'intensité incidente peut être modifiée de façon à la rendre proche d'une forme gaussienne dans le plan de la lentille. Dans ce cas, l'amplitude du champ au point de focalisation sur l'échantillon est également de forme gaussienne. Si le faisceau pompe est de forme spatiale gaussienne, nous obtenons, grâce à la caméra, la répartition spatiale de son intensité de la forme suivante :

$$
I=I_{0} e^{-\frac{x^{2}}{w_{p}^{2}}}
$$

Le faisceau pompe induit dans le matériau une variation d'indice complexe de forme gaussienne :

$$
n=n_{0}+\frac{n_{2}}{2} I
$$

où $\mathrm{n}_{0}$ est l'indice linéaire et $\mathrm{n}_{2}$ l'indice non linéaire. C'est le paramètre que nous essayons de caractériser. Nous l'écrivons sous la forme :

$$
n_{2}=n_{2}{ }_{2}+i n^{\prime \prime}{ }_{2}
$$

$n_{2}^{\prime}$ est lié essentiellement à l'indice de réfraction et $n_{2}{ }_{2}$ est proportionnel à l'absorption.

Le trajet optique dans l'échantillon est dans ce cas $\left(n_{0}+\Delta n\right)$ d. Le faisceau test sonde une variation d'indice $\Delta \mathrm{n}$. Le diamètre du faisceau test est quatre ou cinq fois plus petit que celui 
du faisceau pompe sur l'échantillon. Deux cas peuvent se présenter :

Les deux faisceaux sont focalisés concentriquement sur l'échantillon (encadré de la figure 28), le faisceau test sonde une zone presque homogène autour de l'origine : le sommet de la gaussienne est supposé de forme parabolique :

$$
\Delta n=\Delta n_{0}\left(1-\frac{r^{2}}{w_{p}^{2}}\right)
$$

Dans cette configuration, l'échantillon soumis à l'excitation du faisceau pompe se comporte comme une lentille. Le faisceau test subit une focalisation si $\Delta n>0$ ou une défocalisation si $\Delta \mathrm{n}<0$.

Une seconde configuration permet de déterminer la variation maximale de la partie réelle de l'indice de réfraction, lorsque les faisceaux ne sont pas concentriques sur l'échantillon. Dans cette disposition, le faisceau test sonde une zone spatiale où l'excitation n'est plus homogène et il est dévié. Le cristal se comporte comme un milieu d'épaisseur optique de profil gaussien. L'épaisseur optique du cristal s'écrit :

$$
x=n d=x_{0} e^{-\left(\frac{z}{z}\right)^{2}}
$$

La déviation maximale est obtenue lorsque la position de la tache du test par rapport à celle de la pompe est telle que $\frac{d x}{d z}$ soit maximal. Cette situation correspond au point d'inflexion du profil gaussien au point $z=\frac{Z}{\sqrt{2}}$. Le développement au premier ordre de l'épaisseur optique donne, autour du point d'inflexion :

$$
x=n_{0} d+d \frac{d n}{d z} z
$$

Cette variation linéaire provoque une déviation d'un faisceau d'un angle $\Phi$ donné par la relation :

$$
\tan (\Phi)=d \frac{d n}{d z}
$$

Dans la mesure où l'angle $\Phi$ est petit,

$$
\Phi=\frac{d n}{d z} d \approx \frac{1}{Z} \sqrt{\frac{2}{e}} \Delta n d
$$

d'où

$$
\Delta n=\Phi \frac{Z}{d} \sqrt{\frac{e}{2}}
$$

La variation d'indice est proportionnelle à l'angle de déviation. 


\subsubsection{Dynamique de la variation de profil spatial}

L'étude en fonction du temps de la variation du profil spatial d'un faisceau permet ainsi d'étudier la dynamique des processus entrant en jeu dans les non-linéarités optiques des propriétés. Des temps de déclin de l'ordre de la durée des impulsions ou supérieurs sont déterminés. L'intensité au niveau du cristal est de $8 \mathrm{MW} / \mathrm{cm}^{2}$ pour une durée d'impulsion de 150 ps. Cette excitation créé une densité de paires électron-trou de l'ordre de $10^{17} / \mathrm{cm}^{3}$.

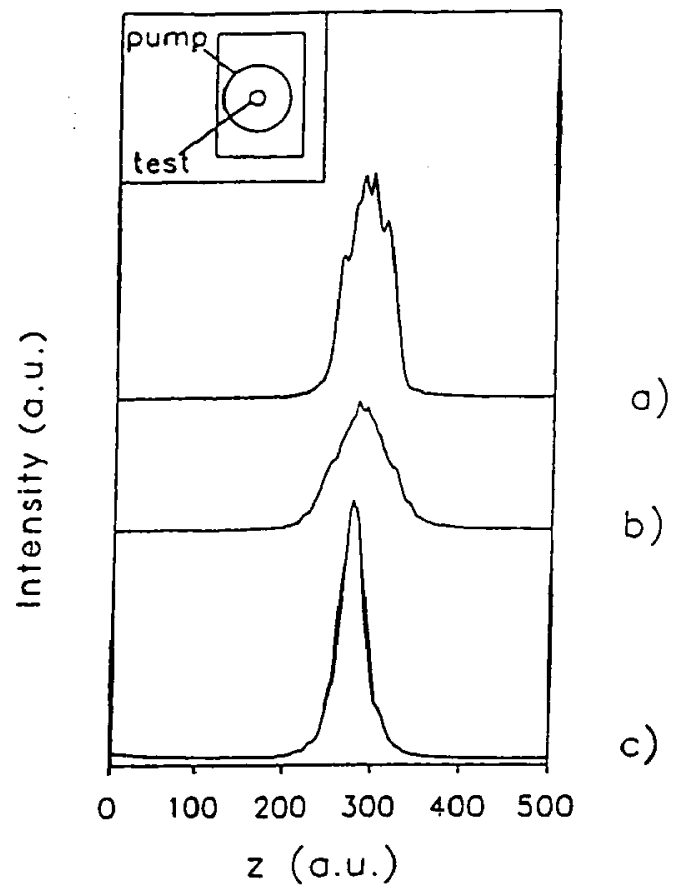

Figure 28 : Modifications spatiales en configuration concentrique. (a) sans excitation, (b) $\tau=3,8 \mathrm{~ns}$ (c) $\tau=12,9 \mathrm{~ns}$.

La figure 28 montre l'évolution temporelle du profil spatial d'un faisceau test. La courbe (a) est la distribution spatiale du faisceau test transmis par l'échantillon sans excitation par le faisceau pompe. Les courbes (b) et (c) présentent les variations de profil à différents instants pour une excitation de $8 \mathrm{MW} / \mathrm{cm}^{2}$. À partir des courbes obtenues, nous pouvons évaluer la variation de transmission différentielle $\gamma$ égale à :

$$
\gamma=\frac{I_{t}-I_{t+p}}{I_{t}}
$$

où $I_{t}\left(I_{t+p}\right)$ est l'intensité sans (avec) excitation et la variation de la taille du faisceau :

$$
\delta=\frac{w_{t+p}-w_{t}}{w_{t}}
$$

où $w_{t}\left(w_{t+p}\right)$ est la largeur du faisceau sans (avec) excitation. Ces quantités sont représentées 
sur les figures 29 et 30 en fonction du retard $(\Delta t)$ du faisceau test par rapport au faisceau pompe. Dès que les deux impulsions commencent à se superposer (figure 29), une focalisation induite du faisceau test est observée après passage dans l'échantillon qui se comporte comme une lentille convergente. Cette réduction du diamètre du faisceau test est rapidement suivie (à partir de $\tau \sim 0$ ) d'une défocalisation (lentille divergente) jusqu'à un retard de 5,6 ns, puis l'échantillon collimate à nouveau le faisceau. À des retards supérieurs à $30 \mathrm{~ns}$, on constate que l'excitation n'a plus d'effet. La focalisation la plus forte a lieu à $\tau=8,0 \mathrm{~ns}$ et l'importante défocalisation à $\tau=2,3$ ns.

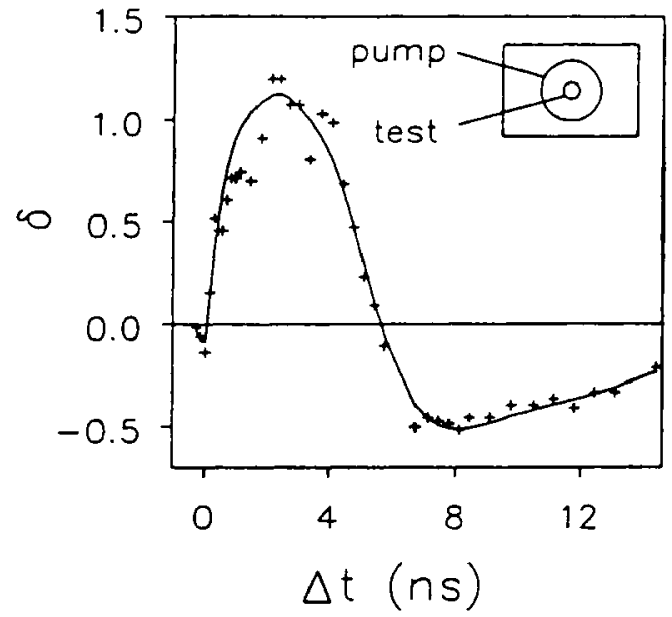

Figure 29 : Variation de la dimension du faisceau test en fonction du retard entre les impulsions des faisceaux d'excitation et de sonde $\delta=\frac{\omega_{t+p}-\omega_{t}}{\omega_{t}}$.

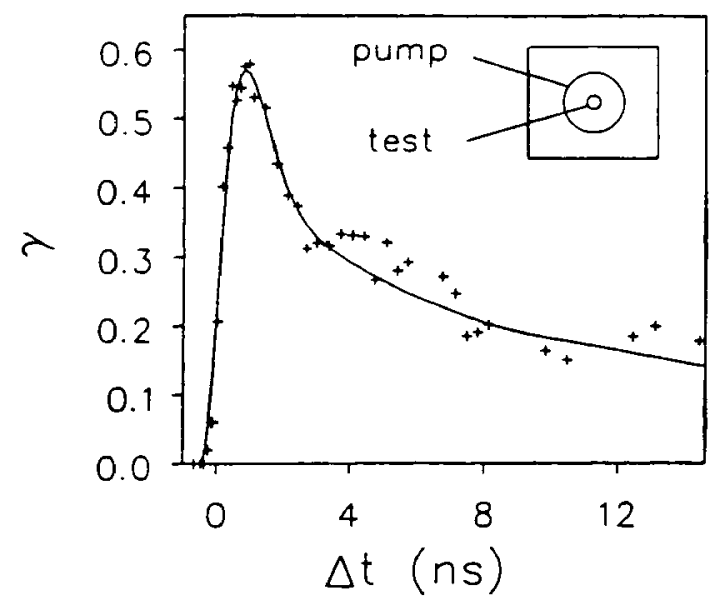

Figure 30 : Transmission différentielle en fonction du retard entre les impulsions des faisceaux d'excitation et de sonde $\gamma=\frac{I_{t}-I_{t+p}}{I_{t}}$. 
La dynamique de l'absorption est très différente. L'absorption induite augmente rapidement jusqu'à $\tau=0,9$ ns pour décroître ensuite exponentiellement comme le montre la figure 30, avec un temps de déclin de $(9,0 \pm 0,5)$ ns. L'absorption induite observée disparaît si l'énergie des photons du faisceau pompe est inférieure à 2,08 eV. Même des excitations plus intenses n'induisent aucun effet dans le matériau à cette énergie de photon.

La figure 31 donne l'évolution temporelle du profil spatial du faisceau test induit par le faisceau pompe lorsque les deux faisceaux ne sont pas concentriques sur le cristal. La courbe (a) correspond à la transmission du faisceau test sans excitation. Sur la courbe (b), on observe une déflection. De plus, une forte absorption induite est également visible.

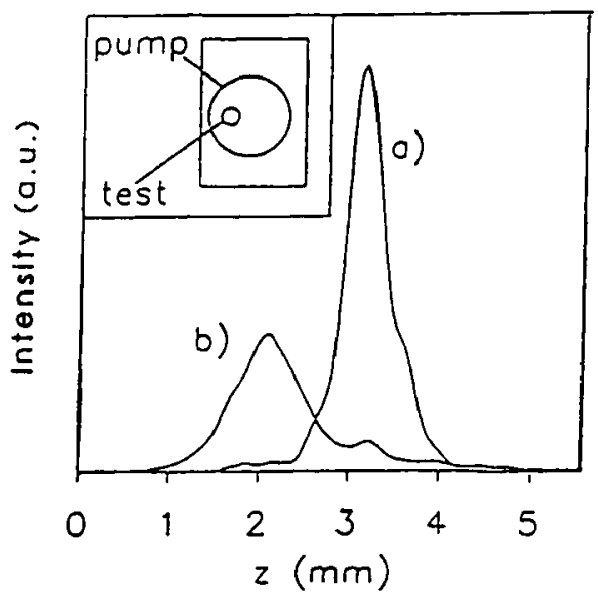

Figure 31 : Profil en configuration non concentrique. (a) sans excitation, (b) $\tau=430 \mathrm{ps.}$

À partir du modèle de la déviation, nous déterminons des déviations de $10^{\circ}$ du faisceau test pour l'échantillon contenant $87 \%$ de zinc pour des intensités de quelques centaines de $\mathrm{kW} / \mathrm{cm}^{2} \mathrm{du}$ faisceau pompe.

À partir de ces mesures, nous obtenons :

$$
\begin{aligned}
& \Delta n_{\text {ch }}^{\prime}=-4,4 \cdot 10^{-19} \mathrm{~cm}^{-3} \\
& \Delta n^{\prime \prime}{ }_{\text {eh }}=2,2 \cdot 10^{-21} \mathrm{~cm}^{-3}
\end{aligned}
$$

Les coefficients non linéaires sont donc très importants dans le tellurure de cadmium et de zinc. Ceci permet d'induire de fortes non-linéarités à des intensités d'excitation faibles comparées à celles obtenues avec de nombreux semi-conducteurs.

Ces études ont été réalisées par des mesures de test-pompe. Il est évident que des changements tellement importants des $n^{\prime}$ donnent lieu à une très forte focalisation ou défocalisation du faisceau pompe et changent profondément les propriétés de propagation des faisceaux dans de tels matériaux (propagation de solitons, par exemple).

\subsection{Z-scan}

Une autre méthode pour déterminer simultanément la variation de la partie réelle et imaginaire de l'indice de réfraction est la méthode de "Z-Scan " où l'échantillon est déplacé par rapport au foyer [39] comme le montre la figure 32. 


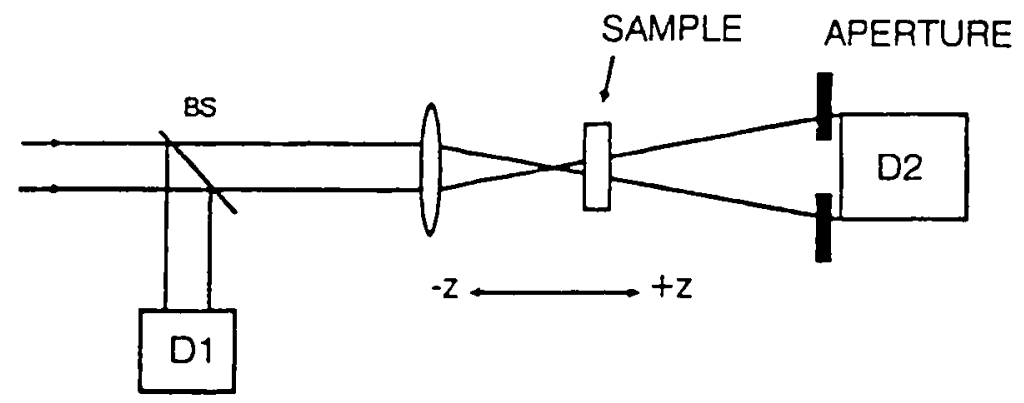

Figure 32 : Montage expérimental de Z-Scan [39].

Le faisceau dont la répartition d'intensité I(r) est de forme gaussienne, est focalisé par une lentille au point $z=0$ de son axe de propagation. Au-delà de ce point, à une dizaine de fois la distance de Rayleigh, une fraction du faisceau est extraite à l'aide d'un diaphragme de faible ouverture. Le Z-Scan consiste à mesurer avec la photodiode D2 la variation de l'intensité du faisceau transmis par le diaphragme en fonction de la position de l'échantillon de part et d'autre du point de focalisation $(z=0)$ du faisceau. Si l'échantillon montre un effet Kerr, $\mathrm{n}$ est de la forme

$$
n=n_{0}+\frac{n_{2}}{2}|E(r)|^{2}
$$

où la distribution radiale du champ électrique $|E(r)|^{2}$ est proportionnelle à $\mathrm{I}(\mathrm{r})$. Si l'échantillon se trouve loin du foyer, l'intensité est faible et son indice de réfraction est spatialement constant. Plus proche du foyer, I devient important et l'échantillon joue le rôle d'une deuxième lentille qui focalise ou défocalise le faisceau, suivant le signe du coefficient de Kerr $\mathrm{n}_{2}$. Ceci déplace le foyer du système lentille/échantillon, et à intensité incidente constante, (qui est mesurée après une lame séparatrice par le détecteur $\mathrm{Dl}$ ) le détecteur $\mathrm{D} 2$ reçoit une intensité plus ou moins grande. Cette intensité transmise par le diaphragme dépend du signe de $n_{2}$ et de la position de l'échantillon par rapport à $z=0$. Pour des faisceaux gaussiens l'intensité transmise par le diaphragme peut être calculée en fonction de $z$ et le résultat est montré sur la figure 33 pour le cas où l'absorption non linéaire est négligeable. Les deux courbes sont obtenues pour $n_{2}<0$ (trait pointillé) et $n_{2}>0$ (trait plein).

$Z_{0}$ est la longueur de diffraction du faisceau qui dépend de la longueur d'onde et du diamètre de la tache de focalisation. Du fait de la focalisation ou de la défocalisation induite discutée précédemment, les courbes sont asymétriques par rapport à $z=0$. Si l'échantillon montre aussi une absorption induite, on peut la déterminer par la même approche en ouvrant le diaphragme. Dans ce cas, le changement d'indice ne joue aucun rôle parce que D2 détecte toujours l'ensemble de la lumière transmise par l'échantillon. Celui-ci absorbe plus quand l'échantillon est au foyer de la lentille (à $\mathrm{z}=0$ ) que quand il est en dehors du foyer.

Comme le montre la figure 34 , l'intensité transmise est une fonction symétrique par rapport à $z=0$. En effectuant des mesures avec (fig. 34b) et sans (fig. 34a) diaphragme, l'absorption induite et le changement d'indice peuvent être déterminés simultanément. 


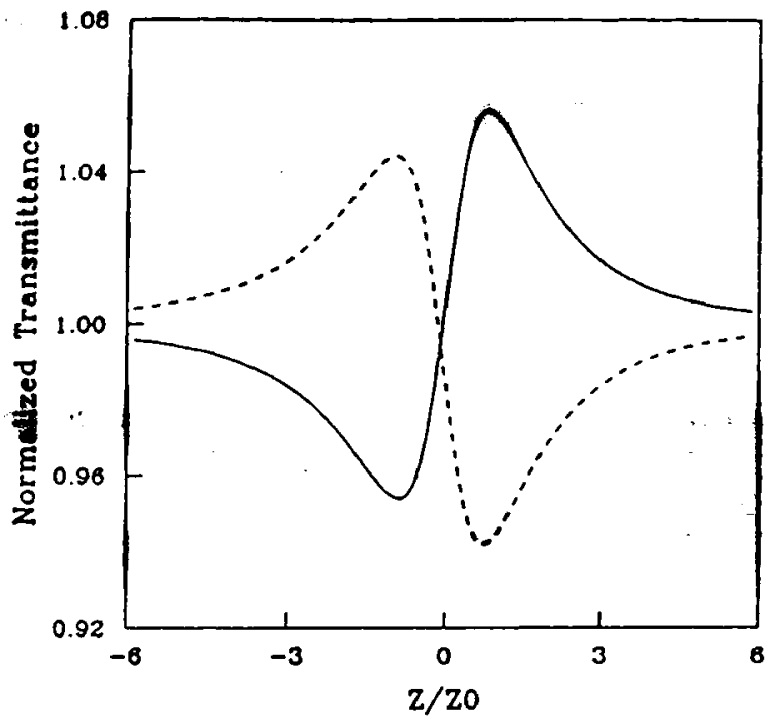

Figure 33 : Transmittance calculé en cas d'une non-linéarité dispersive de Kerr pour le cas $\mathrm{n}_{2}<0$ (trait pointillé) et $\mathrm{n}_{2}>0$ (trait plein).[39].

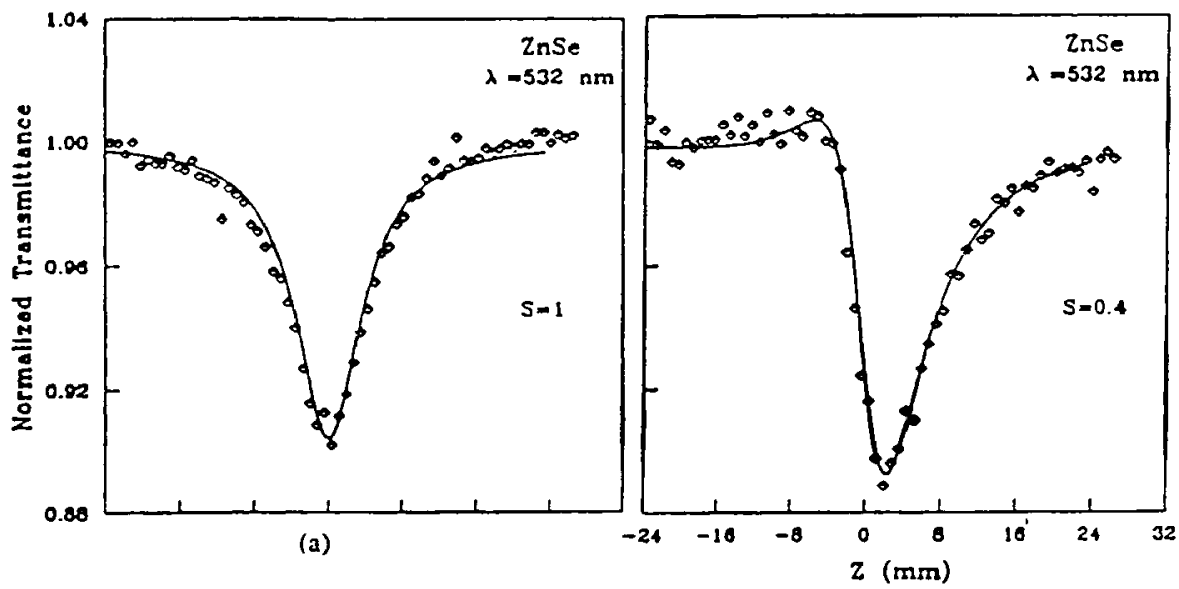

(b)

Figure 34 : Transmittance normalisée de ZnSe déterminée par la technique Z-Scan (a) sans et (b) avec diaphragme. Les traits pleins donnent le résultat pour le meilleur ajustement du calcul [39].

Bien que cette expérience paraisse relativement simple à réaliser du point de vue technique, elle nécessite néanmoins que quelques critères soient impérativement satisfaits : un échantillon homogène et un faisceau laser parfaitement gaussien, dont le profil ne change pas 
d'une impulsion à l'autre. De plus l'analyse théorique des résultats impose que l'on travaille hors du domaine de la saturation des non-linéarités optiques.

\subsection{Transmission induite (spectral hole-burning)}

Selon la configuration électronique du matériau étudié, on peut obtenir une augmentation comme dans le cas du $\mathrm{C}_{60}$ ou une diminution de l'absorption par excitation laser. Si la raie d'absorption est élargie d'une manière inhomogène, une source spectralement fine peut saturer des transitions à l'intérieur de cette bande et on obtient donc une augmentation de la transmission d'un faisceau test [40] (spectral hole-burning). Si la largeur spectrale du faisceau pompe est plus fine que la largeur homogène de la transition, cette dernière peut être déterminée à partir de la largeur spectrale du "trou" (Fig. 35). Cette technique a été largement utilisée pour étudier les transitions dans des gaz mais on peut aussi l'appliquer à l'étude des nanocristallites dans des matrices transparentes [41]. Comme nous l'avons vu déjà, le confinement quantique des électrons et des trous dans des nanocristaux change leur énergie. À cause des fluctuations des dimensions des différents nanocristaux, les raies de transitions excitoniques sont élargies d'une manière inhomogène. Comme nous le verrons, la largeur homogène d'une transition est inversement proportionnelle au temps de cohérence de la quasiparticule qui peut ainsi être déterminé. Nous avons effectué de telles mesures avec des nanocristaux de $\mathrm{CuBr}$ dans une matrice de verre de borosilicate [42-46]. Nous utilisons pour ces mesures des sources impulsionnelles nanosecondes et le montage présenté sur la figure 15.
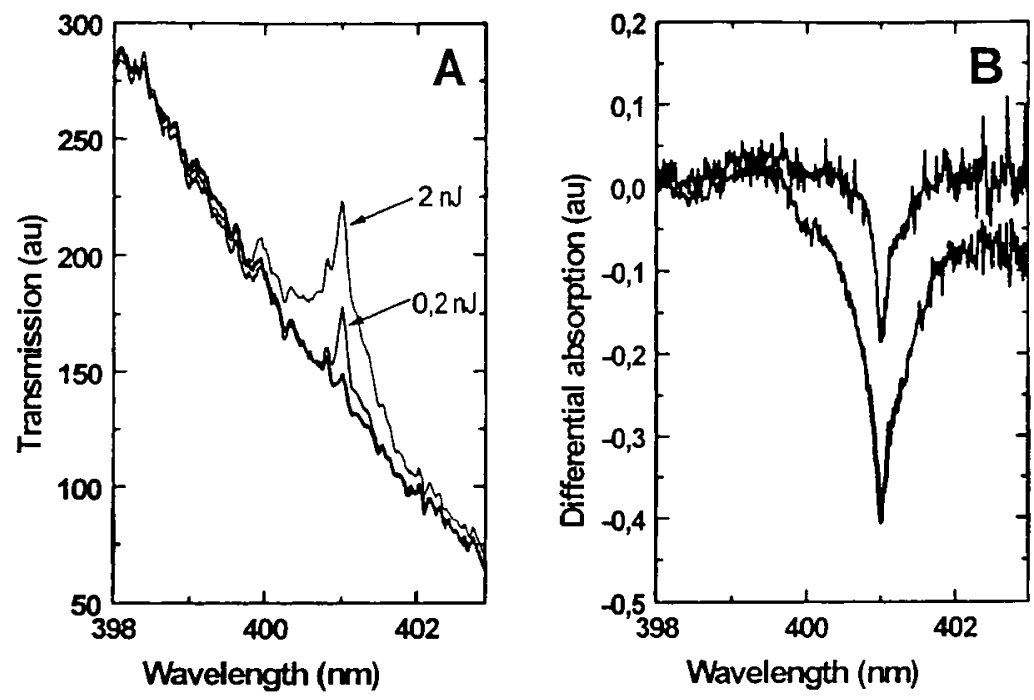

Figure 35 : Spectres de transmission (A) et d'absorption différentielle (B) transitoires de l'échantillon contenant les nanocristaux de $2,8 \mathrm{~nm}$, à $2 \mathrm{~K}$, pour deux énergies du faisceau pompe accordé à $401 \mathrm{~nm}$.

Les mesures que nous présentons ont été effectuées à différentes longueurs d'onde d'excitation et à basse température $(2 \mathrm{~K})$ pour l'échantillon contenant les nanocristaux de 
2,8 $\mathrm{nm}$ de rayon moyen. Le faisceau pompe est issu d'un laser à colorant (PBBO dissout dans du dioxane à $0,4 \mathrm{~g} . \mathrm{l}^{-1}$ ) à cavité à incidence rasante et pompé par le laser à excimères $(\mathrm{XeCl})$ nanoseconde. Nous disposons de cette façon d'une source laser très fine spectralement et accordable dans la raie d'absorption excitonique $Z_{1,2}$ de l'échantillon étudié. Le faisceau test est l'émission collimatée d'une cuve contenant le même colorant, et pompée par le même laser à excimères. L'expérience consiste alors à résoudre spectralement les variations induites sur la transmission du test par la pompe.

\subsubsection{Séparation des effets transitoires et permanents}

Les spectres de hole-burning que nous avons mesurés ont un caractère permanent et transitoire : certains changements induits par la pompe dans la transmission du test perdurent plusieurs minutes après que l'échantillon ait été illuminé par le faisceau laser. Étant du même ordre de grandeur que les effets transitoires, les effets permanents en perturbent la mesure, et il s'avère nécessaire de pouvoir s'en affranchir expérimentalement. La transmission du test à travers l'échantillon est dans ce but mesurée à plusieurs reprises :

Sur une zone de l'échantillon non encore illuminée par le faisceau pompe, la transmission du test est successivement analysée spectralement dans les conditions suivantes :

- en absence du faisceau pompe.

- en présence du faisceau pompe. Comparé au spectre précédent, celui-ci permet de quantifier les effets cumulés des hole-burning permanent et transitoire.

- en absence du faisceau pompe. Cette mesure contient des informations relatives au holeburning permanent.

Ainsi, en soustrayant le spectre de la troisième mesure à celui de la seconde, on obtient le spectre de hole-burming transitoire. Remarquons enfin qu'il est nécessaire de s'assurer que la diffusion de la pompe est suffisamment faible pour pouvoir être négligée.

\subsubsection{Hole-burning transitoire}

La figure 35 présente la transmission du faisceau test, pour deux énergies du faisceau pompe ( 2 et $0,2 \mathrm{~nJ}$ ) par impulsion et sans faisceau pompe, ainsi que les spectres d'absorption différentielle correspondants. Ces spectres sont corrigés des effets du hole-burning permanent.

Les mesures ont été effectuées au deuxième ordre de diffraction du réseau du spectrographe. La résolution spectrale de notre chaîne de détection est alors de $0,2 \mathrm{meV}$ : elle est insuffisante à résoudre le spectre du laser d'excitation dont la largeur à mi-hauteur est inférieure à $0,05 \mathrm{meV}$. C'est donc la détection et non l'excitation qui limite ici la résolution de l'expérience. Nous avons donc systématiquement soustrait $0,2 \mathrm{meV}$ aux largeurs des " trous " creusés dans les spectres de transmission du test, afin de compenser l'élargissement des spectres du fait de la moindre résolution expérimentale.

Chaque "trou" creusé dans le spectre de transmission du test par la pompe peut alors être ajusté par une fonction de forme lorentzienne. La largeur à mi-hauteur $\Gamma_{F W H M}^{\text {Trou }}$ de cette lorentzienne dépend de l'intensité d'excitation I, de la largeur de raie homogène $\Gamma_{F W H M}^{\text {homo }}$ et sature au-delà de l'intensité $I_{s}$ :

$$
\Gamma_{F W H M}^{\text {trou }}=\Gamma_{F W H M}^{\mathrm{hom} o}\left(1+\sqrt{1+I / I_{S}}\right)
$$




$$
\text { ou } \frac{\Gamma_{F W H M}^{\text {bom o }}}{2 \hbar}=\frac{1}{T_{2}}
$$

Ainsi, à intensité nulle, le trou creusé dans les spectres d'absorption a pour largeur le double de la largeur de raie homogène. La figure 36 présente sa variation avec l'intensité d'excitation.

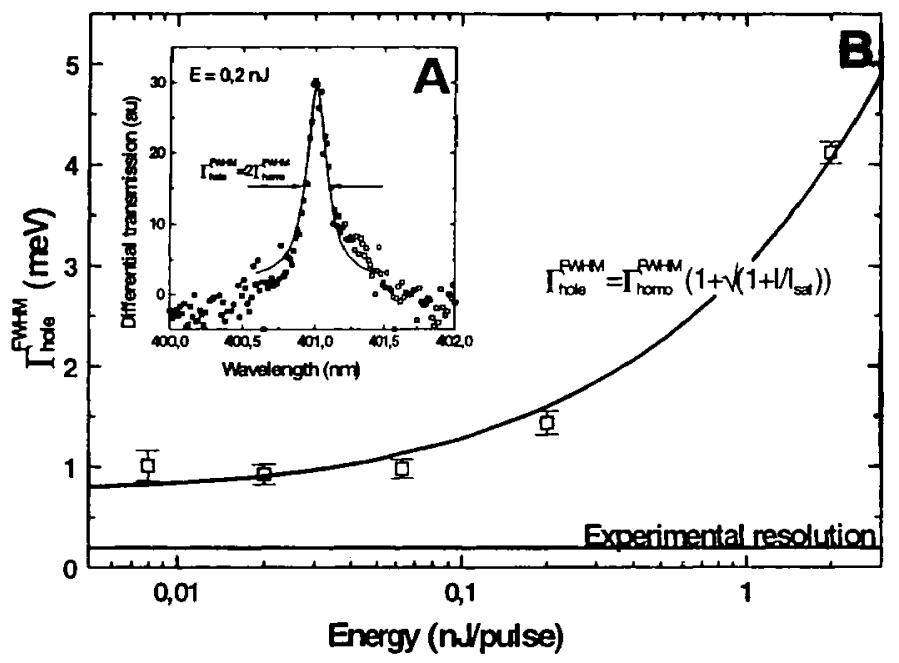

Figure 36 : Ajustement par une lorentzienne d'un spectre d'absorption différentielle (A) et variation de la largeur de cette lorentzienne avec l'énergie d'excitation (B).

L'ajustement des résultats expérimentaux par la formule est représenté en trait plein sur la figure 36. Il donne la valeur $\Gamma_{F W H M}^{\text {homo }}=0,38 \mathrm{meV}$, soit un temps de déphasage $\mathrm{T}_{2}$ de $3,47 \mathrm{ps}$.

Les mêmes mesures de l'évolution des spectres de hole-burning avec l'intensité d'excitation ont été effectuées aux longueurs d'ondes de 395 et $405 \mathrm{~nm}$ dans la raie d'exciton $Z_{12}$. Les spectres mesurés sont ajustés par des lorentziennes. Ces résultats seront comparés à des mesures de $\mathrm{T}_{2}$ par d'autres méthodes (mélange de quatre ondes) plus loin.

\subsubsection{Hole-burning permanent}

Comme nous l'avons déjà mentionné, les spectres de hole-burning que nous avons mesurés ont un caractère transitoire et permanent. Dans la mesure du temps de déphasage la composante permanente de ces spectres a été expérimentalement éliminée. Efle est similaire au photonoircissement et nous allons montrer qu'elle comporte des informations intéressantes et qu'en particulier elle donne une mesure de l'énergie des phonons optiques longitudinaux dans nos échantillons.

Le hole-burning permanent est un phénomène très étudié, notamment du fait de sa possible application dans des systèmes de stockage numérique très haute densité. Si l'inscription des données se fait avec plusieurs longueurs d'onde, il permet d'enregistrer plusieurs données en un même point physique dans la mémoire de stockage. Il est cependant nécessaire pour cela de disposer d'un matériau présentant un élargissement inhomogène très large. 
Par l'expérience acquise avec des matériaux organiques pour cette spectroscopie, les mécanismes conduisant au hole-burning permanent sont habituellement classés en deux catégories : le hole-burning photochimique (traditionnellement noté $P H B$, acronyme de photochemical hole-burning) et le hole-burning photophysique (noté $N P H B$, pour Non PHB). Nous les décrivons ici.

Dans un mécanisme photochimique, les particules photoréactives (molécules, nanocristaux...) subissent des changements chimiques importants. L'absorption optique du produit de cette réaction chimique se fait généralement dans une région spectrale différente de celle des réactifs de départ. Le nouveau spectre d'absorption peut ainsi se trouver fortement déplacé vers les hautes ou vers les basses énergies.

Au contraire, dans le cas du hole-burning photophysique, c'est l'interaction entre les particules photoréactives et leur environnement (solvant, matrice) qui est modifiée par l'interaction avec la lumière. L'absorption du produit n'est que très peu décalée spectralement par rapport à celle des réactifs de départ et l'absorption intégrée sur tout le spectre est identique à celle des réactifs.

L'exploration sur une zone spectrale étendue d'un spectre de hole-burning permanent acquis sur l'échantillon de nanocristaux de $2,8 \mathrm{~nm}$ de rayon moyen montre que les changements de transmission à la position du laser excitateur sont superposés à une absorption induite présentant deux maxima centrés à environ 3,03 et $3,30 \mathrm{eV}$.

La séparation énergétique entre les différentes structures observées étant relativement importante, le mécanisme du hole-burning permanent dans nos échantillons est probablement de nature photochimique. Il est possible que, sous une forte intensité d'excitation, un porteur photo-excité (électron ou trou) soit éjecté en dehors du volume du nanocristal et se trouve piégé soit à l'interface semi-conducteur-matrice, soit dans la matrice elle-même. Le champ électrique statique créé par la séparation de l'électron et du trou peut être la cause d'un effet Stark électro-optique. Ce mécanisme réactionnel aboutit à un produit stable et permet d'expliquer le caractère permanent des spectres de hole-burning de nature photochimique. C'est le mécanisme généralement envisagé dans la littérature. Il devient de plus en plus probable lorsque la taille des nanocristaux diminue, la barrière de potentiel à l'interface entre le semi-conducteur et la matrice diminuant lorsque l'énergie de confinement augmente.

\section{PHOTOLUMINESCENCE ET AMPLIFICATION OPTIQUE DUES À DES PROCESSUS DE COLLISION}

Dans le paragraphe 2 nous avons déjà introduit les biexcitons comme nouvelles quasiparticules $[1,29]$ qui peuvent être excitées par absorption à deux photons. Ils peuvent relaxer sur leur courbe de dispersion et se recombiner radiativement par exemple en une b.i.p. de caractère excitonique et une autre b.i.p. de caractère photonique. Cette demière est observée comme émission de l'échantillon appelée "luminescence". Dans le cas des biexcitons, cette recombinaison a lieu avec une conservation d'énergie et de la quantité de mouvement (équation (33)). Dans la figure 8, les raies de luminescence $\mathbf{N}$ ont pu être identifiées à ce processus de recombinaison de biexcitons qui ont une faible énergie cinétique à cause de leur création par absorption résonante de deux photons.

Dans cet exemple, des b.i.p. de caractère excitonique sont créées en grande densité. Elles peuvent se coupler à deux et, par émission d'un phonon optique, créér de nouveau un biexciton qui possède une importante énergie cinétique. Ceci est dû au fait que l'énergie et le vecteur d'onde de l'ensemble des quasi-particules impliqués dans le processus doit être 
conservé. Les biexcitons possèdent une masse effective deux fois plus grande que celle des excitons. Ils relaxent et se thermalisent en émettant des phonons acoustiques et se recombinent à nouveau en b.i.p. de caractère excitonique avec un grand vecteur d'onde et une b.i.p. de caractère photonique et de faible vecteur d'onde. Si l'on compare ce processus avec celui donnant lieu à la raie d'émission $\mathrm{N}$, on remarque que l'énergie cinétique des biexcitons initiale est plus grande et leur distribution sur leur courbe de dispersion beaucoup plus large. Parce que excitons et biexcitons ont des masses effectives différentes, la raie d'émission que l'on obtient ainsi (nommée " $M$ ") est spectralement large. La forme spectrale de cette raie (forme "Boltzmann inverse") reflète la distribution en énergie des biexcitons et la densité d'état des bandes. Les recombinaisons radiatives des biexcitons en exciton longitudinal et b.i.p. de caractère photonique est aussi possible et des raies " $\mathrm{M}_{\mathrm{L}}$ " et " $\mathrm{N}_{\mathrm{L}}$ " sont ainsi observées. Parce que b.i.p. et excitons longitudinaux (figure 2) ont des énergies différentes, les énergies de photon des raies $N_{L}$ et $N$ ainsi que des raies $M_{L}$ et $M$ sont séparées de $\left(E_{L}-E_{0}\right)$.

$\mathrm{Si}$ l'on excite les excitons en résonance, les raies $\mathrm{N} n$ ' apparaissent pas parce que tous les biexcitons sont créés avec une grande énergie cinétique. Normalement, on identifie ces raies à partir de leur position spectrale si les énergies d'exciton et de biexciton sont connues (par exemple à partir des études d'absorption à deux photons et de Hyper-Raman dans le cas de $\mathrm{CuCl}$ ). Leur intensité varie quadratiquement avec l'intensité d'excitation avant de montrer généralement une saturation. Leur spectre d'excitation montre des résonances sur l'énergie d'exciton $E_{0}$ et sur $E_{B} / 2$.

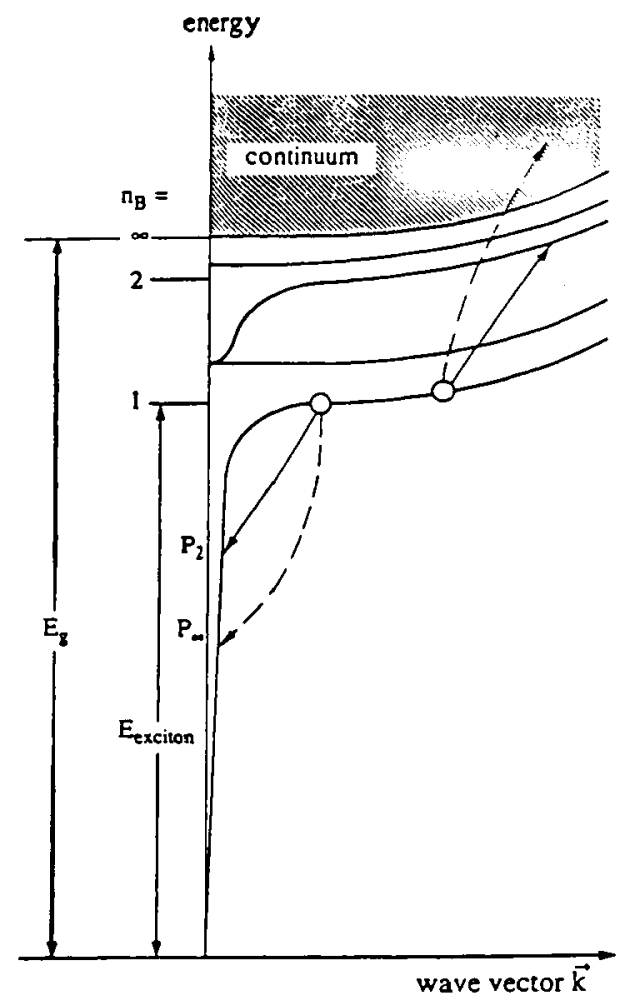

Figure 37 : Schéma de processus de collision exciton-exciton. 
A part la recombinaison des biexcitons, d'autres raies apparaissent à forte intensité d'excitation. Des raies $P$, par exemple sont dues à des processus de collision exciton-exciton [47-51]. Comme le montre le processus $P_{2}$ de la figure 37, en obéissant aux lois de conservation d'énergie et de la quantité de mouvement, un b.i.p. de caractère excitonique change son vecteur d'onde et devient photonique. Un deuxième exciton passe de l'état fondamental (nombre quantique principal $n=1$ ) à l'état $n=2$ de la série de Rydberg de l'exciton. À plus hautes intensités d'excitation, un processus similaire est observé où l'état final du deuxième exciton est un état du continuum électron-trou (l'exciton est dissocié). Cette dernière raie d'émission est habituellement appelée $P_{\infty}$. Parce que la forme et position spectrales de ces raies dépend de la distribution initiale des excitons, ils changent avec la température et l'intensité d'excitation.

Un processus similaire est schématisé sur la figure 38 pour la collision exciton-électron (ou exciton-trou) [52-54]. Dans ce cas, la distribution des excitons et des électrons qui sont créés optiquement intervient. À faible densité d'électrons, le processus donne lieu à une asymétrie de la raie de recombinaison excitonique qui se déplace vers de faibles énergies de photon quand on augmente l'intensité.

Lorsque des excitons-polaritons existent, le plasma d'électrons ou de trous est non dégénéré et ils obéissent à une distribution de Boltzmann. La forme spectrale de ces raies est comme dans le cas des raies $\mathbf{P}$ une forme "Boltzmann inversée " mais de largeurs différentes parce qu'électrons, trous et excitons ont des masses effectives différentes [52, 53].

Comme dernière raie de collision qui est souvent observée, je mentionne la raie de collision exciton piégé-électron (ou trou) $[55,56]$. Ces raies prennent naissance aux basses énergies de photon des raies de recombinaison d'un exciton, piégé par un donneur ou accepteur. Contrairement aux autres processus, ils ne font pas intervenir la conservation de la quantité de mouvement parce que l'impureté a brisé l'invariance sous translation du système. Ces raies saturent rapidement à forte intensité d'excitation due à la petite densité des impuretés.
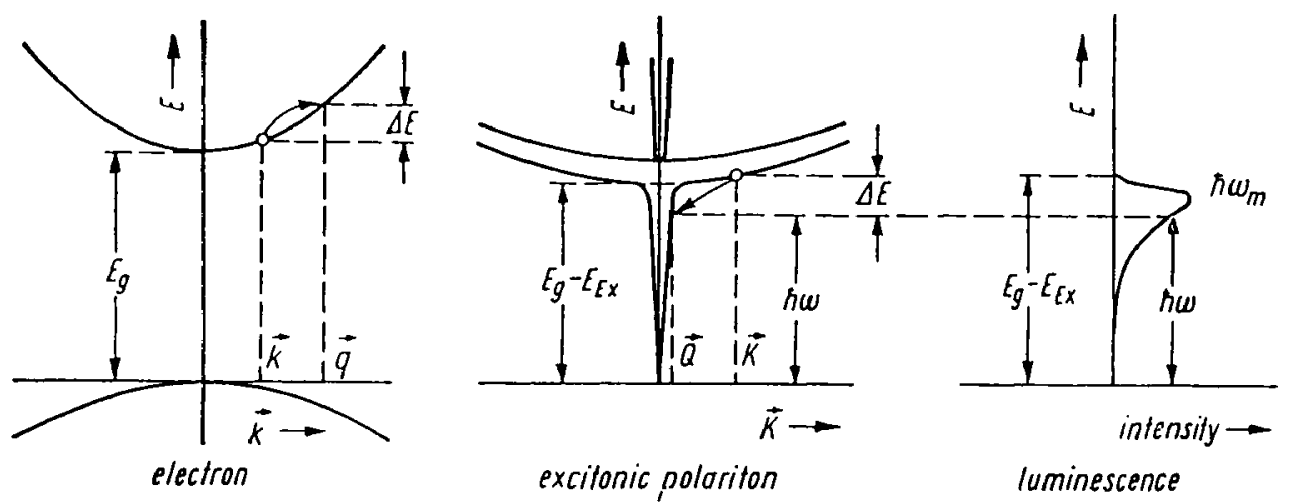

Figure 38 : Schéma de processus de collision exciton-électron.

Les processus de collision font intervenir au moins deux quasi-particules et trois états différents du système. Ils peuvent donc montrer de l'amplification optique ou du " gain ". Ceci est dû au fait que le coefficient d'absorption $\alpha_{i j}^{\prime}$ pour des transitions résonantes entre un niveau $\mathrm{j}$ vers un niveau i est proportionnel à 


$$
\alpha_{i j}^{r} \propto W_{i j} \frac{\omega^{2} \Gamma^{2}}{\left[\left(\omega_{i}-\omega_{j}\right)^{2}-\omega^{2}\right]^{2}+\Gamma^{2} \omega^{2}}\left(n_{j}-n_{i}\right)
$$

$W_{i j}$ est proportionnel à la probabilité de transition et $n_{j}$ et $n_{i}$ donnent les probabilités que les niveaux $\mathrm{j}$ et $\mathrm{i}$ sont occupés. Ceci est un résultat de la mécanique quantique et signifie que si la transition $\mathrm{j} \rightarrow \mathrm{i}$ (absorption) est possible, $\mathrm{i} \rightarrow \mathrm{j}$ (émission) est aussi possible et la probabilité qu'une transition a lieu doit être proportionnelle à la probabilité que l'état initial est occupé. En conséquence, si $\mathrm{n}_{\mathrm{j}}<\mathrm{n}_{\mathrm{i}}$ et $\alpha_{i j}^{r}<0$, on peut obtenir une émission stimulée et une amplification optique [40]. Pour la réponse linéaire, seulement l'état fondamental $g$ (avec $\left.\omega_{g}=0\right)$ est occupé $\left(n_{g}=1\right.$ pour $\left.T=0\right)$ et tous les $n_{i} \equiv 0$. Dans ce cas l'équation $(52)$ se réduit à l'équation $(21)$.

Considérons l'exemple où un laser excite en résonance des biexcitons. Dans ce cas, on a à faire à un système à trois niveaux, l'état fondamental ( $g$ ), l'exciton (j) et le biexciton (i). Parce que les biexcitons sont excités en résonance, $n_{i}>n_{j}$ et $\left(\left(n_{j}-n_{i}\right)\right.$ et $\left.\alpha_{i j}^{r}\right)<0$. Si nous écrivons l'absorption totale sous la forme

$$
\alpha=\alpha_{i j}^{r}+\tilde{\alpha}=-G
$$

où $\widetilde{\alpha}$ représente des pertes supplémentaires non résonantes, et $\alpha=-G$ peut devenir $<0$. Dans ce cas, d'après la loi de Beer-Lambert, l'intensité transmise $I_{t}$ par un échantillon de longueur $L$ est donnée par

$$
I_{1}=I_{0} e^{-\alpha d}
$$

où $\mathrm{I}_{\mathrm{o}}$ est l'intensité incidente. $\mathrm{I}_{\mathrm{t}}$ augmente donc d'une manière exponentielle et nous obtenons une amplification optique $[47,57]$. L'amplification optique peut par exemple être observée dans des mesures de luminescence ou de diffusion Hyper Raman en transmission.

Une autre configuration [58] a été développée par Shaklee (encadré dans la figure 39) : l'excitation à longueur de ligne variable (variable stripe length). Dans ce cas, dans la partie centrale homogène d'un faisceau, une partie rectangulaire est sélectionnée par deux fentes. Une lentille cylindrique focalise le faisceau sous forme d'un trait de typiquement $30 \mu \mathrm{m} \times 2000 \mu \mathrm{m}$ sur le bord de l'échantillon. Un masque mobile permet de faire varier la longueur $\mathrm{L}$ du trait. Pendant l'excitation, si l'émission spontanée se propage dans la direction longue $\mathrm{z}$ du trait, elle est amplifiée. Comme le montre la figure 39 , son intensité dépend de l'intensité d'excitation.

L'équation différentielle pour l'intensité I d'émission est donnée par

$$
\frac{d l}{d z}=I G+I_{s p}
$$

où $\mathrm{I}_{\mathrm{sp}}$ est l'intensité spontanée par unité de longueur. L'équation (54) a comme solution

$$
I(L)=I_{s p} \frac{e^{G L}-1}{G}
$$




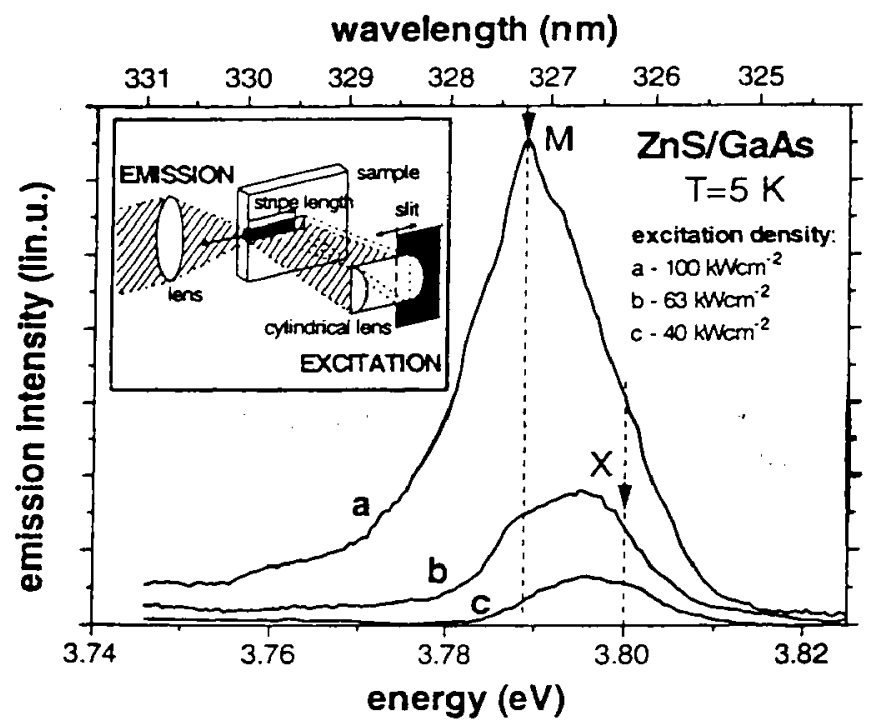

Figure 39 : Spectre d'émission d'un film de $\mathrm{ZnS}$ excité par un laser à $\mathrm{XeCl}$ (à $308 \mathrm{~nm}$ ) à $\mathrm{L}=1,2 \mathrm{~mm}$ et à des intensités différentes. $\mathrm{X}$ et $\mathrm{M}$ indiquent les positions spectrales des raies d'émission des excitons libres et des biexcitons. L'encadré montre le schéma de montage de la méthode de longueur de trait variable.

L'émission est normalement analysée par un spectromètre [59-61] et le coefficient de gain $G$ peut être déterminé en mesurant l'intensité émise pour des différentes longueurs $L$ et en analysant (figure 40) le résultat avec l'équation (55).

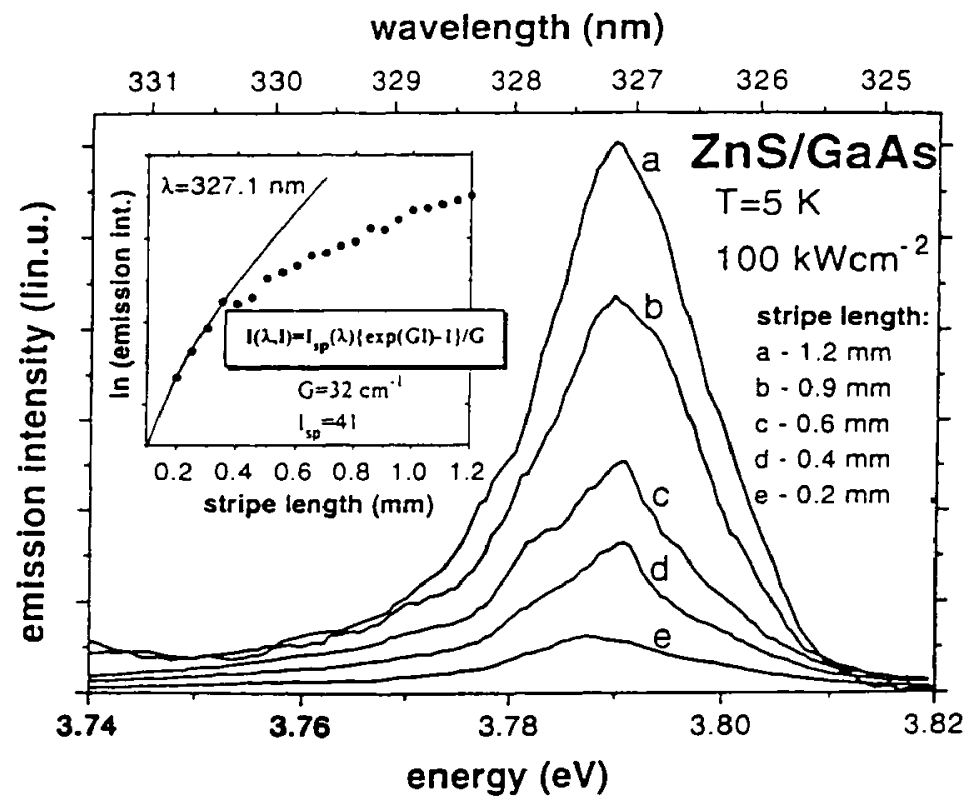

Figure 40 : Spectre d'émission d'un film de $\mathrm{ZnS}$ excité avec une intensité de $100 \mathrm{~kW} / \mathrm{cm}^{2}$ à des longueurs de trait variable. L'encadré montre la détermination du gain $\mathbf{G}$ (équation 51 ). 
Cette méthode est très élégante parce qu'elle permet de déterminer $\mathrm{G}$ et la longueur à partir de laquelle l'émission spontanée amplifiée sature sans que l'on soit obligé de construire une cavité autour de l'échantillon ou de l'utiliser comme milieu actif d'un amplificateur optique.

\section{RÉSEAUX INDUITS PAR LASER}

Nous avons vu dans ces derniers chapitres que l'excitation d'un échantillon par un champ électromagnétique peut provoquer une réponse non linéaire. Ainsi, son indice de réfraction et/ou son coefficient d'absorption sont modifiés. Cet effet est utilisé dans les expériences de réseaux induits par laser [62] ou, plus généralement, dans les expériences de mélange à 4 ondes $[63,64]$. Pour expliquer le schéma général de cette technique, nous considérons dans un premier temps une impulsion lumineuse cohérente qui traverse un séparateur de faisceaux et est ainsi divisée en deux faisceaux $P_{1}$ et $P_{2}$ de vecteurs d'ondes $\vec{k}_{1}$ et $\vec{k}_{2}$ d'intensité $I_{1}$ et $I_{2}$ et de même longueur d'onde $\lambda_{\mathrm{p}}$. Les impulsions sont focalisées sur un échantillon. Lorsqu'ils sont en coïncidence temporelle et spatiale à la surface de l'échantillon, ils y forment une figure d'interférences qui n'est autre qu'une modulation spatiale périodique de l'intensité lumineuse dans le milieu. Cette modulation est maximale si $\mathrm{I}_{1}=\mathrm{I}_{2}$ et dans ce cas l'intensité totale I varie périodiquement entre 0 et $4 \mathrm{I}_{1}$. $\grave{A}$ cause de la non-linéarité, le milieu présente également une variation spatiale périodique de son indice complexe de réfraction et il se comporte comme un réseau de diffraction. Le vecteur $\vec{Q}$ du réseau et son pas $\Lambda$ sont fonctions de l'angle $\theta$ que font entre elles les deux impulsions incidentes :

$$
\begin{gathered}
\left.\vec{Q}= \pm\left(\vec{k}_{1}-\vec{k}_{2}\right) \text { (par convention, on choisit dans la suite } \vec{Q}=\vec{k}_{2}-\vec{k}_{1}\right) \\
\Lambda=\frac{2 \pi}{|\vec{Q}|}=\frac{\lambda_{P}}{2 \sin (\theta / 2)} \approx \frac{\lambda_{P}}{\theta} \text { si } \theta \ll 1
\end{gathered}
$$

Le réseau est induit par une variation de l'indice complexe de réfraction. Si cette variation est due à une variation de l'indice réel, on parle de réseau de phase. Au contraire, on parle de réseau d'amplitude lorsqu'elle est due à une variation de l'absorption. La présence de l'un de ces deux cas est déterminée par l'énergie de photons excitateurs :

- s'ils sont en résonance avec une transition électronique du milieu, l'absorption varie fortement et l'indice réel très peu : on est dans le cas d'un réseau d'amplitude.

- s'ils sont décalés par rapport à la résonance, la variation d'indice. complexe est essentiellement due à une variation d'indice réel : c'est un réseau de phase.

Du fait de la variation périodique spatiale, le vecteur d'onde d'une quasi-particule n'est défini qu'à $m \cdot \vec{Q}$ près où $m$ est un entier. Ainsi, lorsqu'un faisceau $\mathrm{P}_{3}$ de vecteur d'onde $\vec{k}_{3}$ et d'intensité $\mathrm{I}_{3}$ teste le réseau de diffraction de vecteur $\vec{Q}$, il s'y diffracte, à l'ordre m dans la direction $\vec{k}_{4}$ donnée par la relation :

$$
\vec{k}_{4}=\vec{k}_{3}+m \cdot \vec{Q}
$$


Nous verrons que $\vec{k}_{4}$ doit de préférence être le vecteur d'onde caractérisant un état propagatoire. La relation (58) est connue comme "condition de Bragg " en physique du solide ou "accord de phase" en optique non linéaire. De plus la conservation d'énergie (équation. 33) impose une condition supplémentaire sur les modules des vecteurs d'ondes à cause de la relation de dispersion (équations. 17 et 33).

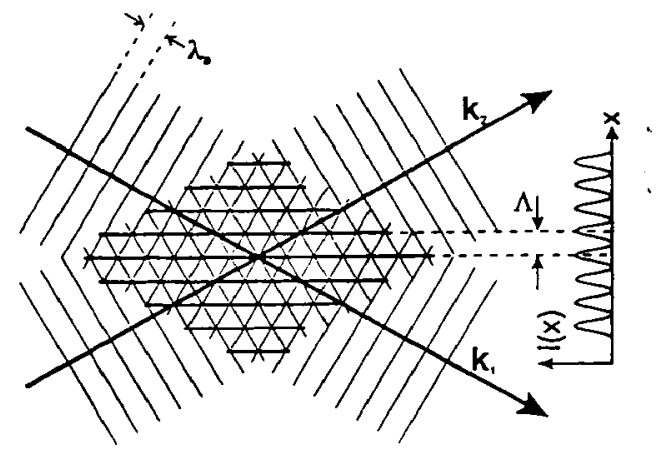

Figure 41 : Inscription d'un réseau de diffraction dans le volume d'un échantiłlon.

Comme le montre la figure 41 , les franges d'interférence sont créées dans le volume de l'échantillon. Pour une incidence normale [62] d'un faisceau test, diffracté sur ce réseau, il existe un déphasage $\varphi$ entre une onde partielle diffractée dans l'angle $\phi_{1}$ à l'entrée de l'échantillon et celle diffractée dans la même direction après que le test s'est propagé de la longueur $\mathrm{d}$ dans le milieu. Le déphasage est donné par

$$
\varphi=\frac{2 \pi}{\lambda_{t}} n \cdot \Delta l
$$

où $\lambda_{\mathbb{l}} / \mathrm{n}$ est la longueur d'onde du faisceau test dans le matériau d'indice $\mathrm{n}$ et $\Delta \mathrm{l}$ la différence des deux chemins. On obtient alors

$$
\varphi=\frac{2 \pi}{\lambda_{1}} n\left(\frac{d}{\cos \left(\phi_{1}\right)}-d\right)
$$

Si l'on considère $\mathrm{d}$ comme épaisseur de l'échantillon, les ondes partielles sont émises dans la direction $\alpha$ telle que

$$
\sin \alpha / \sin \phi_{1}=n
$$

et interfèrent constructivement à l'infini si $\sin \alpha=\mathrm{m} . \lambda_{\mathrm{N}} / \Lambda$ où $\mathrm{m}$ est un entier. Si nous considérons $m=1$, l'équation (61) donne l'angle pour une interférence constructive :

$$
\sin \phi_{1}=\frac{\lambda_{t}}{n \Lambda}
$$

si $\phi_{1} \ll 1$ est petit, nous pouvons développer les équations (60) et (62) et obtenons

$$
\varphi=\frac{\pi \lambda_{t} d}{\Lambda^{2} n}
$$

Pour que ces interférences soient constructives, il faut que $\varphi \approx 0$ donc il faut inscrire des réseaux de grand pas sur des échantillons aussi fins que possible. Le déphasage est alors minime, et la condition $\varphi \ll<1$ est appelée condition des réseaux fins, ou encore condition de Raman-Nath. 
Si $\varphi>\sim 1$, le réseau est appelé " épais " ou " réseau de volume ".

Lorsque ce déphasage est important, les différentes ondes diffractées interfèrent de façon destructive : aucun signal ne peut être observé. Une interférence constructive, ou le déphasage $\varphi=0$ pour toutes les ondes partielles sortant de l'échantillon, peut être obtenue dans le cas où l'angle d'incidence $\beta$ du test est choisi tel que $\beta=-\alpha$. Dans cette configuration symétrique, tous les chemins optiques des différentes ondes partielles diffractées sont égaux et ils interfèrent d'une manière constructive

Le mélange à quatre ondes peut de cette façon être compris dans l'interaction de quatre faisceaux de vecteurs d'onde $\vec{k}_{i}(i=1,2,3$ ou 4$)$ : les deux premiers inscrivent un réseau de diffraction sur lequel un troisième se diffracte pour générer le quatrième.

Considérons $\varphi=0$ dans le cas limite où les faisceaux pompes ne sont pas atténués. Si nous considérons que des processus de troisième ordre, un faisceau $\mathrm{P}_{4}$ est généré qui est caractérisé par le vecteur d'onde

$$
\vec{k}_{4}=\vec{k}_{2}-\vec{k}_{1}+\vec{k}_{3}+\Delta \vec{K}
$$

$\vec{k}_{4}$ doit être un état propagatoire vérifiant la relation de dispersion. Dans l'équation (60) $\Delta \vec{K}$ est le désaccord de phase qui peut apparaître parce que la conservation d'énergie des quatre quasi-particules doit être strictement respectée. Son intensité $\mathbf{L}_{4}$ est de la forme

$$
I_{4} \propto\left|\chi^{(3)}\right|^{2} I_{1} I_{2} I_{3} d^{2}\left(\frac{\sin (\Delta K d / 2)}{\Delta K d / 2}\right)^{2}
$$

Considérons le cas où il y a "accord de phase" ( $\Delta \mathrm{K} \mathrm{d}=0)$ qui donne lieu à la plus grande intensité de diffraction. L'efficacité de diffraction est définie par

$$
\eta=\frac{I_{4}}{I_{3}}
$$

où $\mathrm{I}_{3}$ est l'intensité du faisceau diffracté sur le réseau créé par les faisceaux " 1 " et “ 2 ”. $\eta$ peut être calculé analytiquement dans les approximations discutées plus haut.

Une modulation spatiale de l'indice de réfraction complexe $\tilde{n}=n+\Delta \tilde{n}(x)$ mène à une amplitude de la transmittance $t(x)$ de la forme :

$$
\mathrm{t}(\mathrm{x})=\mathrm{t}_{0} \mathrm{e}^{\mathrm{i} \phi \cos (2 \pi x / \Lambda)} \quad \text { avec } \quad \phi=\frac{2 \pi \Delta \tilde{n} d}{\lambda}
$$

Si $\mathrm{A}_{\mathrm{i}}$ est l'amplitude de l'onde incidente, l'amplitude $\mathrm{A}_{\mathrm{m}}$ de l'onde diffractée à l'ordre $\mathrm{m}$ est donnée par :

$$
A_{m}=\frac{A_{i}}{\Lambda} \int_{0}^{\Lambda} e^{i[\phi \cos (2 \pi x / \Lambda)+2 \pi m x / \Lambda]} d x
$$

En faisant le changement de variables $t=2 \pi x / \Lambda$, il vient : 


$$
\begin{aligned}
A_{m} & =\frac{A_{i}}{2 \pi} \int_{0}^{2 \pi} e^{i \phi \cos t} \cos (m t) d t \\
\because & =A_{i} i^{m} J_{m}(\phi)
\end{aligned}
$$

où $\mathrm{J}_{\mathrm{m}}$ est la fonction de Bessel d'ordre $\mathrm{m}$. Pour $\phi<<\mathrm{l}$,

$$
\begin{aligned}
& J_{0}(\phi) \approx 1 \\
& J_{1}(\phi)=J_{-1}(\phi) \approx \phi / 2
\end{aligned}
$$

Parce que $\phi \ll 1$, l'efficacité de diffraction au premier ordre $\eta$ vaut alors :

$$
\eta=\frac{I_{S}}{I_{T}}=\left|\frac{A_{1}}{A_{i}}\right|^{2}=\left|\frac{\pi \Delta \tilde{n} d}{\lambda}\right|^{2}
$$

soit avec $\Delta \tilde{n}=\Delta n+i \Delta \xi$ :

$$
\eta=\left|\frac{\pi \Delta n d}{\lambda}\right|^{2}+\left|\frac{\pi \Delta \xi d}{\lambda}\right|^{2}
$$

Pour une non-linéarité de type Kerr :

$$
\tilde{n}_{2} I=\Delta \tilde{n}=\frac{\chi^{(3)}}{\varepsilon_{0} n^{2} c} \sqrt{I_{1} I_{2}}
$$

et nous obtenons :

$$
\eta=\frac{I_{4}}{I_{3}}=\frac{\pi^{2} d^{2}\left|\chi^{(3)}\right|^{2}}{\lambda^{2} \varepsilon_{0}^{2} c^{2} n^{4}} I_{1} I_{2}
$$

À partir des équations (67) et (73) on peut donc déterminer $\chi^{(3)}$ en mesurant les intensités des quatre faisceaux.

\subsection{Réseaux épais}

Si la longueur d'interaction $d$ est grande, $\Delta \mathrm{K}$ doit être approximativement nul et la condition de Bragg s'impose. La configuration la plus simple [65] à deux faisceaux, est montrée sur la figure 42

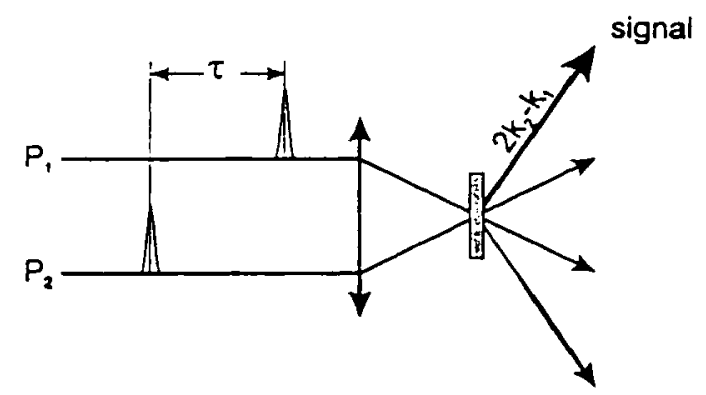

Figure 42 : Expérience de mélange à quatre ondes dans une configuration à deux faisceaux, destinée à la mesure du temps de déphasage. 
Deux impulsions, appelées $P_{1}$ et $P_{2}$ émergeant de la même source sont focalisées sur un échantillon (des nanocristallites de $\mathrm{CuCl}$ ici). $\mathrm{Si} \mathrm{I}_{1} \ll \mathrm{I}_{2}$, à coïncidence temporelle $(\tau=0$ ), les deux impulsions créent, comme décrit plus haut, une modulation spatiale de l'indice de réfraction et la pompe $P_{2}$ se diffracte dans la direction de $P_{1}$. La condition de Bragg est remplie et, $s$ 'il l'on compare avec la figure 41 , la configuration est symétrique. Les ondes partielles diffractées sont donc toutes en phase et interfèrent d'une manière constructive, dans la direction de $P_{1}$. On obtient donc un transfert d'intensité du faisceau $P_{2}$ vers $P_{1}$. Si l'on augmente l'intensité de $P_{1}$, l'intensité transférée diminue et pour $I_{1}=I_{2}$ le faisceau " $P_{1}$ " transfère autant d'intensité vers " $P_{2}$ " comme " $P_{2}$ " vers " $P 1$ ".

On voit très bien (figure 42) qu'également d'autres faisceaux sont générés dans les directions $2 \vec{k}_{1}-\vec{k}_{2}$ et $2 \vec{k}_{2}-\vec{k}_{1}$. Ils ne remplissent pas, par contre, la condition d'accord de phase et leur intensité est négligeable dans le cas des réseaux épais. Le vecteur d'onde de l'état propagatoire $\vec{k}_{4}=2 \vec{k}_{2}-\vec{k}_{1}+\Delta \vec{K}$ fait intervenir un $\Delta \vec{K} \neq 0$ dans le cas dégénéré parce que $\left|\vec{k}_{4}\right|=\left|\vec{k}_{1}\right|=\left|\vec{k}_{2}\right|$. Si l'on écrit la polarisation d'ordre trois $\mathrm{P}_{\mathrm{nl}}$ pour la configuration de transfert d'énergie, et que l'on considère les termes se propageant dans la direction de $\vec{k}_{1}$, elle prend la forme :

$$
P_{n l}=A\left(E_{2} E_{1}^{*}\right) E_{2}+B\left(E_{2} E_{2}\right) E_{1}^{*}+C I_{P_{2}} E_{1}^{*}
$$

où les grandeurs $\mathrm{A}, \mathrm{B}$ et $\mathrm{C}$ sont proportionnelles à $\chi^{(3)}$. Le premier terme correspond à la création du réseau avec le vecteur du réseau $\vec{k}_{r}= \pm\left(\vec{k}_{2}-\vec{k}_{1}\right)$. Sur celui-ci un photon du faisceau $\mathrm{P}_{2}$ se diffracte, donnant lieu au transfert d'énergie. Le deuxième terme décrit une absorption à deux photons de $\mathrm{P}_{2}$, suivie d'une recombinaison induite par le champ $\mathrm{E}_{1}$. D'après l'équation (58), elle se propage également dans la direction $\vec{k}_{1}$ [65]. Ce terme est important dans $\mathrm{CuCl}$ lorsque les biexcitons sont excités d'une manière résonante par $\mathrm{E}_{2}$. Les deux termes décrivent des processus cohérents. Le troisième, incohérent, décrit le fait que l'impulsion $\mathrm{P}_{2}$ peut saturer l'absorption linéaire des nanocristaux (comme nous l'avons discuté dans le chapitre du "hole burning") et augmente ainsi la transmission du faisceau test.

Si l'on retarde une impulsion par rapport à l'autre, on peut observer la dynamique de ces processus. Le résultat est donné sur la figure 43 . La courbe "a" donne la contribution de la diffraction de la pompe sur le réseau, "b" la recombinaison induite, et "c" la saturation de l'absorption. "d" est la somme des trois processus.

Avec accord de phase, en gardant la configuration symétrique, on peut diffracter une troisième impulsion $P_{3}$ sur le réseau généré par $P_{1}$ et $P_{2}$. Parce que l'on mesure l'émission dans une direction (qu'on filtre spatialement par un diaphragme) suivant laquelle, en absence de $P_{1}, P_{2}$ ou $P_{3}$, on ne détecte pas de lumière, le terme de saturation d'absorption n'a pas d'importance. De plus, dans cette configuration, on a un très bon rapport signal/bruit. Pour cette raison, habituellement, $P_{3}$ n'est pas dans le plan donné par $P_{1}$ et $P_{2}$, ce qui implique que la direction de $\mathrm{P}_{4}$ est bien différente de celle des trois autres faisceaux.

Une autre configuration souvent utilisée est celle de la "conjugaison de phase" [66]. Dans ce cas, deux faisceaux dégénérés se propageant en sens inverse excitent un échantillon avec des surfaces planes et parallèles. Une troisième y est focalisée avec un certain angle. Différents réseaux sont ainsi créés et les photons générés se propagent dans la direction inverse du troisième faisceau. Ce faisceau signal peut être analysé après une lame séparatrice. En changeant les polarisations des différents faisceaux, les différents éléments du tenseur de la 
susceptibilité peuvent être étudiés séparément. En retardant l'impulsion “ 3 " par rapport aux deux autres on obtient des informations sur la dynamique de la non-linéarité [66].

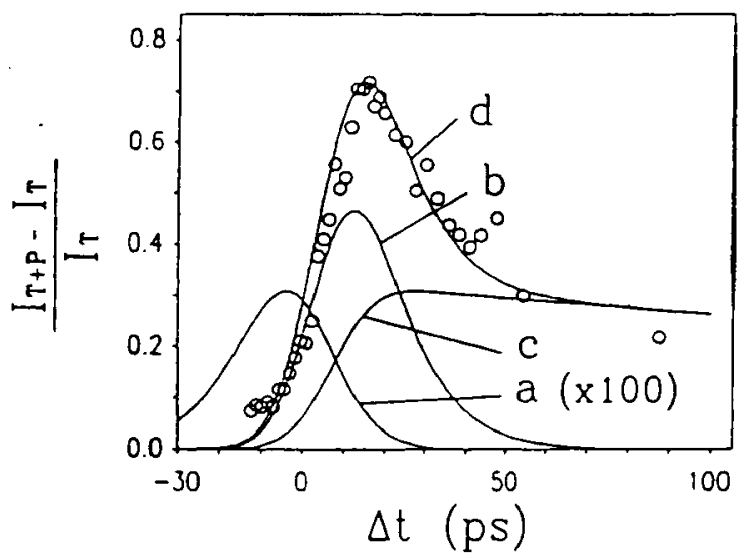

Figure 43 : Différence des intensités des impulsions " test" transmises normalisée avec ou sans excitation par la pompe en fonction du retard $\Delta t$ entre les deux impulsions $(a, b, c, d$ donnant les contributions de différents termes - voir texte).

\subsection{Réseaux fins, expériences à deux faisceaux résolus en temps}

Considérons le cas où $\Delta \mathrm{K} \mathrm{d} \ll 1$ et $\varphi \ll 1$ (équation 63).

Le premier processus qui perturbe la vie d'une paire électron-trou photocréée est la perte de la cohérence de la polarisation associée au champ excitateur, décrite par le temps caractéristique $\mathrm{T}_{2}$. Elle peut être déterminée en utilisant des impulsions cohérentes et de courtes durées excitant le matériau. Si l'on se limite aux effets non linéaires d'ordre 3, les termes cohérents sont donnés par les deux premiers termes de l'équation (75). Si les impulsions sont retardées l'une par rapport à l'autre comme indiqué figure 42 , le début de la première impulsion avec son champ $E_{1}$ créé une polarisation $P_{1}$ qui est cohérente avec $E_{1}$. Après passage de cette impulsion, la polarisation décroît en amplitude et se déphase avec le temps caractéristique $T_{2}$. Quand la deuxième impulsion arrive, son champ $E_{2}$ ne peut interférer qu'avec la partie de la polarisation créée par la première impulsion qui a gardé sa cohérence. Un réseau est créé dont la modulation dépend du retard entre les deux impulsions. Sur ce réseau, la deuxième impulsion s'autodiffracte et donne lieu à un signal émis dans la direction $2 \vec{k}_{2}-\vec{k}_{1}$ (figure 42 ). Parce que $\Delta \mathrm{Kd} \ll \mathrm{l}$ ce signal est encore important, même si l'accord de phase n'est pas réalisé. Le signal est grand lorsque l'impulsion " 1 " arrive avant “ 2 ” sur l'échantillon. L'intensité du signal $I_{\mathbf{s}}$ (free induction decay) décroît selon

$$
I_{S} \propto I_{0} e^{-2 t / T_{2}}
$$

où $T_{2}$ représente le temps de cohërence de la polarisation, induit par $\mathrm{P}_{1}$.

Le deuxième terme dans l'équation 75 est de qualité différente : il décrit l'absorption à deux photons de l'impulsion " 2 ". Si, comme par exemple dans $\mathrm{CuCl}$, des biexcitons y sont 
créés dans un état caractérisé par le vecteur d'onde $\vec{K}=2 \vec{k}_{2}$, le champ " 1 ", arrivant plus tard, peut induire leurs recombinaisons. Ceci donne lieu à un signal, également émis dans la direction $2 \vec{k}_{2}-\vec{k}_{1}$. Mais dans ce cas, le signal est grand lorsque l'impulsion " 2 " arrive avant " 1 ". I montre aussi une décroissance exponentielle avec une constante de temps $\tau_{3}$. Dans ce cas, $\tau_{3}$ signifie le temps de cohérence de l'état du biexciton. Nous avons étudié ces deux processus dans $\mathrm{CuCl}$ [67] résolu en temps. La figure 44 montre le montage.

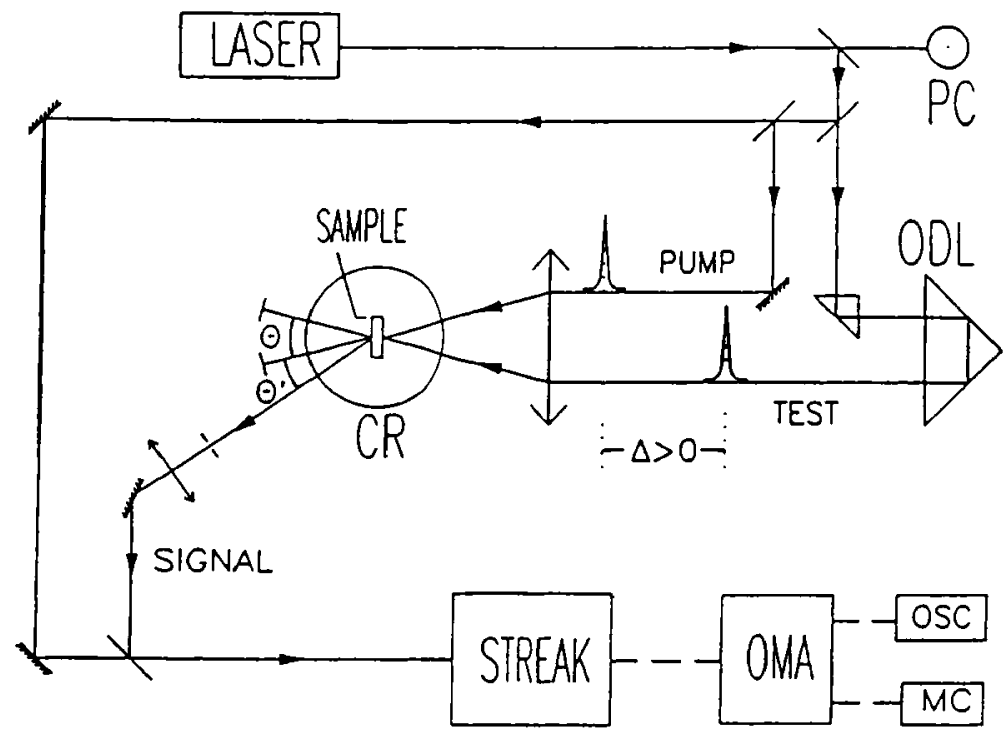

Figure 44 : Configuration expérimentale " en avant ", ou par "transmission " à deux faisceaux provenant de la même source laser, utilisée pour la réalisation du MDQO. Laser : laser à colorant picoseconde accordable ; PC : photocellule pour réaliser la fenêtre d'énergie ; ODL : ligne à retard optique variable ; CR : cryostat ;

Streak : caméra à balayage de fente ; OMA : analyseur optique multicanal ; OSC : oscilloscope ;

MC : micro-ordinateur.

Dans une configuration par transmission et dégénérée, deux faisceaux monoimpulsionnels de même fréquence excitent des échantillons minces de $\mathrm{CuCl}$ de $20 \mu \mathrm{m}$ d'épaisseur dans un cryostat à $4 \mathrm{~K}$.

Le faisceau laser est séparé en deux parties : la plus intense est appelée le faisceau " pompe" et l'autre le " test". Leur rapport d'intensité est fixe, variant entre 8 et 4 selon les séries expérimentales.

L'impulsion du faisceau test peut être retardée ou avancée par rapport à celle du faisceau pompe, grâce à un retard optique variable commandé par un moteur pas-à-pas. Le signal créé est observé à travers l'échantillon dans une direction bien définie, donnée par $2 \vec{k}_{2}-\vec{k}_{1}$. Ce faisceau est tout d'abord diaphragmé, puis rendu parallèle. Ensuite, après un deuxième diaphragme, il est focalisé sur la fente d'entrée du spectromètre ou de la caméra à balayage de fente (streak camera). Afin de réduire le "jitter" inhérent à notre système de détection, nous enregistrons, en même temps que le signal, une fraction de l'impulsion pompe (cf. figure 44). Celle-ci sert à définir l'origine temporelle des événements. 
L'utilisation d'une configuration par transmission nous impose d'étudier des échantillons de faible épaisseur, de manière à minimiser les effets de l'absorption linéaire. Sur la figure 45 sont représentés les signaux lumineux jusqu'au deuxième ordre, obtenus dans le processus de diffusion cohérente quand on excite le cristal mince de $\mathrm{CuCl}$ à la résonance biexcitonique ( $\left.\hbar \omega_{\mathrm{p}}=\mathrm{E}_{\mathrm{bj}} / 2=\hbar \omega_{\mathrm{t}}\right)$. Hors résonance, des signaux jusqu'à l'ordre \pm 5 peuvent être observés. À la résonance biexcitonique, due à une très forte absorption non linéaire, le nombre d'ordres observables décroît.

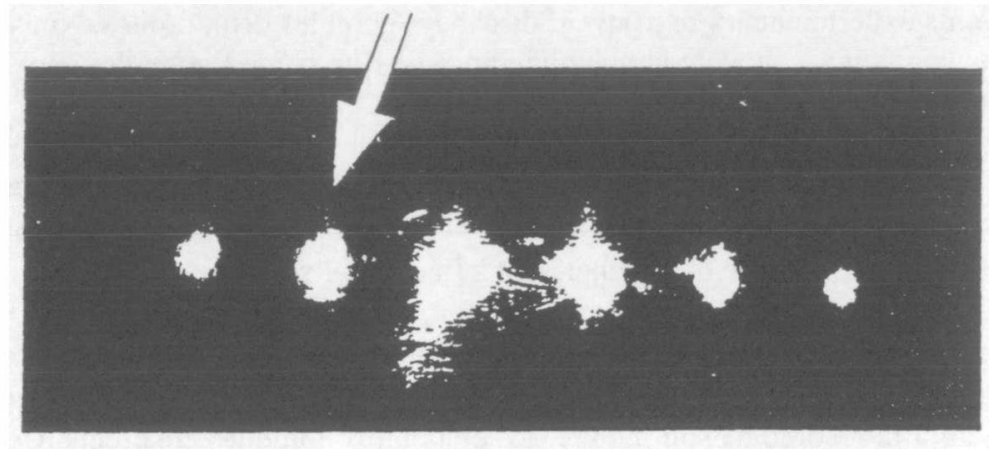

Figure 45 : Figure de diffraction obtenue dans le mélange dégénéré à quatre ondes réalisé avec deux faisceaux dans la configuration "en avant ". La flèche sur la photographie indique le signal diffracté à l'ordre +1 étudié par la suite.

L'évolution temporelle du signal est analysée en enregistrant sa position temporelle par rapport au faisceau pompe, sa forme temporelle et son intensité en fonction du retard $\Delta=t_{t}-t_{p}$, entre les maxima des impulsions pompe $\left(\mathrm{t}_{\mathrm{p}}\right)$ et test $\left(\mathrm{t}_{\mathrm{t}}\right)(\Delta<0$ si l'impulsion test arrive avant l'impulsion pompe sur l'échantillon)

La figure 46 donne la position temporelle du maximum de l'impulsion signal par rapport à celui de l'impulsion pompe $\tau_{\mathrm{M}}=\mathrm{t}_{\mathrm{s}}-\mathrm{t}_{\mathrm{p}}$, mesurée en fonction du retard $\Delta$, quand on excite $\mathrm{CuCl}$ à la moitié de l'énergie du biexciton $(\hbar \omega=3,186 \mathrm{eV})$ et pour une intensité d'excitation $\mathrm{I}_{\mathrm{P}}=5 \mathrm{MW} / \mathrm{cm}^{2}$. Nous comparons les points expérimentaux (croix) aux positions calculées à partir du modèle théorique décrivant les deux processus [68] : recombinaison induite des biexcitons (trait plein) et réseau transitoire induit (tirets).

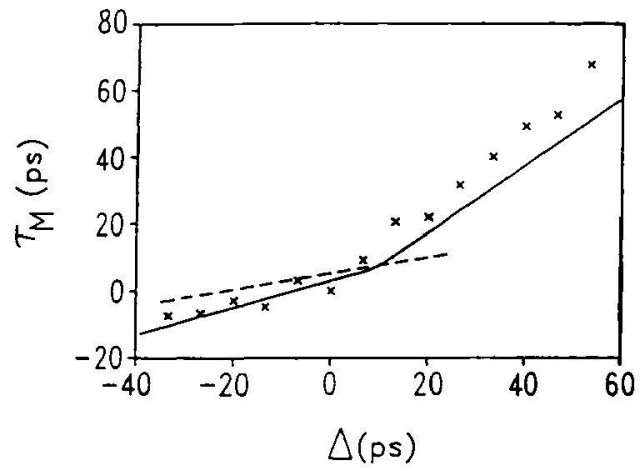

Figure 46 : Position temporelle $\tau_{M}$ du maximum du signal en fonction du retard $\Delta$, à la résonance biexcitonique : ( $x$ ) valeurs expérimentales pour $\hbar \omega=3,186 \mathrm{eV}$ et $\mathrm{I}_{\mathrm{p}}=5 \mathrm{MW} / \mathrm{cm}^{2} ;(\ldots)$ courbe théorique pour le processus de recombinaison induite ; (--- ) courbe théorique pour le processus de réseau transitoire induit. 
Le comportement temporel du signal généré peut être interprété de la manière suivante :

- Pour $\Delta<0$, le signal généré par la recombinaison induite du biexciton suit le recouvrement instantané des impulsions pompe et test. Il en résulte approximativement une droite donnée par l'équation : $\tau_{M} \approx 0,3 \Delta$ (trait plein).

Le signal dû à la diffraction de l'impulsion pompe par le réseau suit l'équation $\tau_{M}=0,25 \Delta$, comme le montre la ligne en tirets.

Les points expérimentaux se trouvent distribués entre les deux droites. Nous ne pouvons donc pas conclure au sujet de la prépondérance de l'un ou de l'autre des deux processus à partir de ces mesures.

- Pour $\Delta>0$ par contre, les positions calculées pour les deux processus sont bien séparées, étant donné que pour la recombinaison induite des biexcitons, $\tau_{M}$ suit asymptotiquement la ligne droite obéissant à l'équation $\tau_{M}=\Delta$.

Ce changement de pente s'explique de la façon suivante : tandis que, pour $\Delta<0$, l'intensité du signal est uniquement une fonction du recouvrement des deux impulsions, pour $\Delta>0$, il est dû à la recombinaison induite des états biexcitoniques cohérents. Ceux-ci, crés par la pompe, conservent leur vecteur d'onde de création, jusqu'à ce que leur recombinaison soit induite par l'impulsion test. Comme on le voit sur la figure 46 , les points expérimentaux suivent la droite de pente 1 , à partir de $\Delta=15 \mathrm{ps,} \mathrm{ce} \mathrm{changement} \mathrm{ayant} \mathrm{lieu} \mathrm{plus} \mathrm{tôt} \mathrm{pour} \mathrm{les}$ intensités d'excitation plus fortes.

Les figures $47 \mathrm{a} / \mathrm{b}$ montrent la trace de corrélation du signal généré [47]. Elle est obtenue en calculant, pour chaque retard $\Delta$, l'intensité intégrée en temps du signal généré. Elles sont obtenues sous excitation résonante $(\hbar \omega=3,186 \mathrm{eV})$, à des intensités d'excitation différentes. Pour $\Delta>15$ ps, d'après la discussion précédente, nous nous attendons à ce que l'intensité du signal soit due uniquement à la recombinaison induite des biexcitons. Elle doit décroître exponentiellement avec une constante de temps $\tau_{3}$ pour les biexcitons. L'asymétrie en $\Delta$ est d'autant plus prononcée que l'intensité d'excitation décroît. L'ajustement, dans cette région de $\Delta$, de la courbe calculée (ligne en pointillé) avec les points expérimentaux (croix) permet d'obtenir la valeur de la constante de temps $\tau_{3}$.

On constate que $\tau_{3}$ varie de $12 \mathrm{ps}$ à $27 \mathrm{ps}$ lorsque l'intensité d'excitation diminue. On vérifie que la décroissance est d'autant plus rapide que l'intensité d'excitation est plus forte, c'est-à-dire que la densité de population et les probabilités de collision augmentent, provoquant la perte du vecteur d'onde de création.

Comme indiquée par la ligne en tiret, la partie montante de la trace de corrélation est surtout due à la contribution de la diffraction de la pompe sur le réseau transitoire. Cette courbe est légèrement asymétrique, son maximum étant situé à $\Delta=-4 \mathrm{ps}$. L'asymétrie est due à des effets de saturation durant l'excitation : le maximum de la modulation du coefficient d'absorption a lieu avant le maximum de l'impulsion pompe. La largeur à mi-hauteur est donnée par la durée de l'impulsion laser.

Les valeurs maxima des deux courbes en tiret et en pointillé sont ajustées de façon que leur somme (courbe en trait plein) donne une déconvolution des résultats expérimentaux.

Si l'on excite le cristal hors de la résonance biexcitonique, l'asymétrie est différente de celle observée précédemment. On observe une augmentation lente de l'intensité du signal suivie d'une décroissance rapide pour $\Delta>0$, due à la saturation de la susceptibilité 

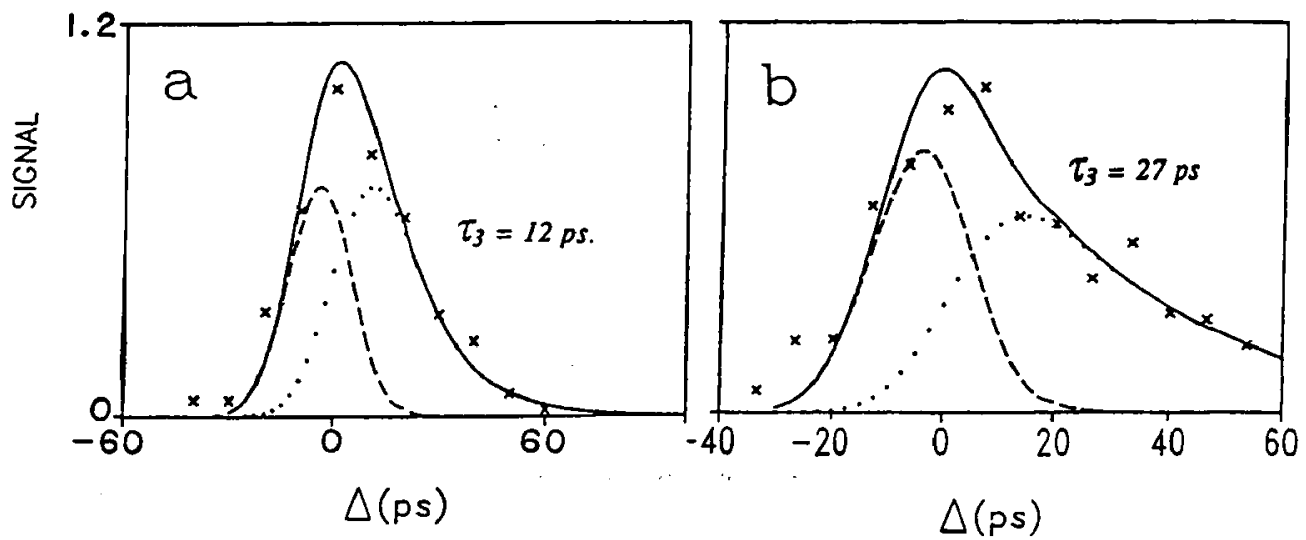

Figure 47 : Intensité intégrée en temps du signal cohérent en fonction du retard $\Delta$, à la résonance biexcitonique. a) sous forte intensité d'excitation : $I_{p}=110 \mathrm{MW} / \mathrm{cm}^{2} I_{t}=I \mathrm{p} / 8, \tau_{3}=12 \mathrm{ps}$. b) à faible intensité d'excitation : $I_{p}=5 \mathrm{MW} / \mathrm{cm}^{2} I_{t}=I_{p} / 4, \tau_{3}=27 \mathrm{ps}$. ( $\mathrm{x}$ ) points expérimentaux ; ( -- ) diffraction de l'impulsion pompe sur le réseau transitoire ; ( ... ) recombinaison induite par l'impulsion test.

non linéaire, conduisant à l'effacement de la modulation spatiale de l'index de réfraction complexe. Ce comportement est caractéristique de la contribution due au réseau.

Nous avons discuté les mesures de mélange à quatre ondes dégénéré avec une double résolution temporelle : le retard entre test et pompe modifie la modulation du réseau et ainsi l'intensité du signal généré. On peut déterminer également l'instant auquel ce signal est émis. Pour des raies de transitions qui montrent un élargissement homogène comme c'est le cas dans le $\mathrm{CuCl}$ massif étudié ici, on observe un "free induction decay" de la polarisation. La constante de temps est alors donnée par $2 \mathrm{~T}_{2}$. Si la raie est élargie d'une manière inhomogène, comme par exemple dans le cas des nanocristaux de $\mathrm{CuBr}$ que nous avons discuté précédemment, on observe alors un écho de photon. Dans un système à deux niveaux, la décroissance du signal est aussi exponentielle [40] mais la constante de temps est donnée par $4 T_{2}$. Ceci est dû au fait qu'une première impulsion courte et ainsi spectralement large créé des polarisations microscopiques qui oscillent à cause de l'élargissement inhomogène à des fréquences propres différentes. Ceci donne lieu à une interférence destructive entre ces polarisations. Ce processus est partiellement réversible [40]. On peut le démontrer en appliquant, après un intervalle temporel $\tau$, une deuxième impulsion qui change la phase de chacun des oscillateurs de $\pi$. Ainsi, après $2 \tau$, les oscillateurs se retrouvent en phase et donnent lieu à une polarisation macroscopique $\mathrm{P}$. Ce signal est détecté comme "écho" de photon. À son intensité participent tous les oscillateurs qui sont restés cohérents avec leurs états de création par la première impulsion. Cette partie décroît avec la constante de temps $T_{2}$ et parce que l'intensité du signal est proportionnelle à $|P|^{2}$, l'écho émis à l'instant $2 \tau$ décroît avec la constante de temps de $4 \mathrm{~T}_{2}$.

Il a été intéressant de mesurer les constantes de temps par mélange de quatre ondes dans des nanocristaux de $\mathrm{CuBr}$ [45] et de comparer les résultats avec ceux obtenus par "spectral hole burning ". Nous utilisons les mêmes échantillons que précédemment et les excitons avec des impulsions d'un laser $\mathrm{Ti}$ : saphir d'une durée de $100 \mathrm{fs}$ dans la région de la résonance excitonique. La configuration est celle de la figure 42 et le signal émis est intégré en temps par un photomultiplicateur. 
La mesure du temps $T_{2}$ de déphasage se fait par extrapolation à intensité nulle des temps de décroissance mesurés pour plusieurs intensités des faisceaux incidents, afin de s'affranchir de l'effet d'intensité pour la détermination du temps de déphasage intrinsèque. Nous présentons ici ces mesures pour l'échantillon contenant les nanocristaux de la plus petite taille $(\mathrm{A}=2,8 \mathrm{~nm})$.

Les dynamiques du signal en fonction de l'intensité des faisceaux incidents sont présentées sur la figure 48. À forte intensité d'excitation, le signal décroît très vite, du fait de la forte densité de porteurs créés. Lorsqu'on diminue l'intensité d'excitation, la densité de porteurs ou des phonons créés par l'excitation diminue d'autant : les processus de déphasage par collisions entre porteurs deviennent moins probables et le temps de décroissance du signal devient plus long.

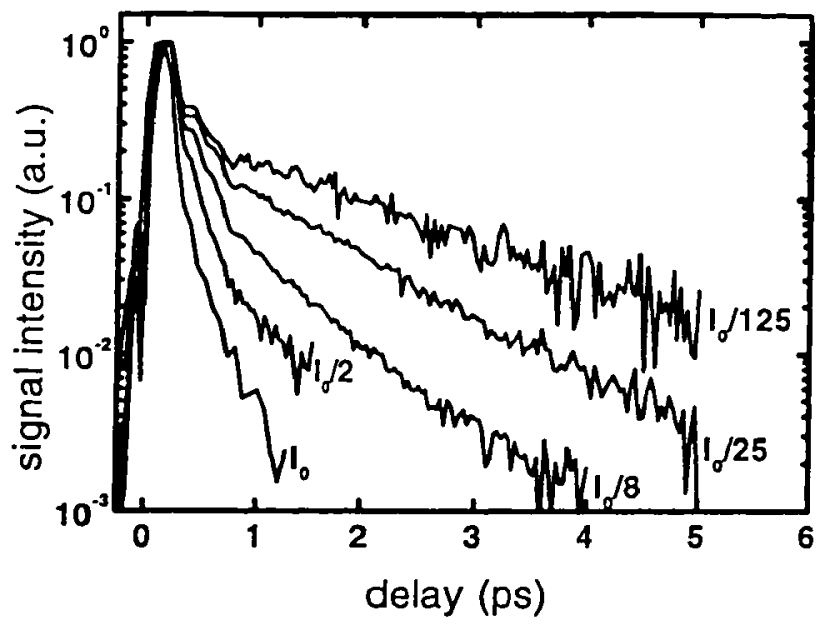

Figure 48 : Signal de mélange à quatre ondes en fonction du retard entre les impulsions pompes, pour différentes intensités d'excitation, à $400 \mathrm{~nm}(3,091 \mathrm{eV})$.

Toutes ces décroissances sont ajustables, pour les retards supérieurs à 500 fs, par des fonctions exponentielles décroissantes :

$$
y_{0}+A e^{-\tau / \tau_{D}}
$$

où $y_{0}$ est le niveau du bruit, $\tau$ le retard entre les deux impulsions, $\tau_{D}$ la constante de décroissance lente et $\mathrm{A}$ l'amplitude associée à cette décroissance.

La figure 49 représente l'évolution du temps $T_{2}=4 \tau_{\mathrm{D}}$. Étant donné que les transitions sont élargies de façon inhomogène dans nos échantillons, la mesure de ce temps long nous donne une mesure de $T_{2} / 4$. Cependant, du fait de l'effet d'intensité décrit avant, il nous faut extrapoler les mesures de $\tau_{\mathrm{D}}$ à intensité nulle pour obtenir une valeur du temps de déphasage intrinsèque du matériau :

$$
\frac{1}{\tau_{D}}=\frac{4}{T_{2}}+\beta I
$$

L'ajustement selon l'équation 77 des résultats expérimentaux est présenté en trait plein sur la figure 49. L'extrapolation à intensité nulle du temps long de décroissance nous donne alors la valeur $T_{2}=7 \mathrm{ps}$, pour l'échantillon contenant les nanocristaux de $2,8 \mathrm{~nm}$ de rayon moyen. 


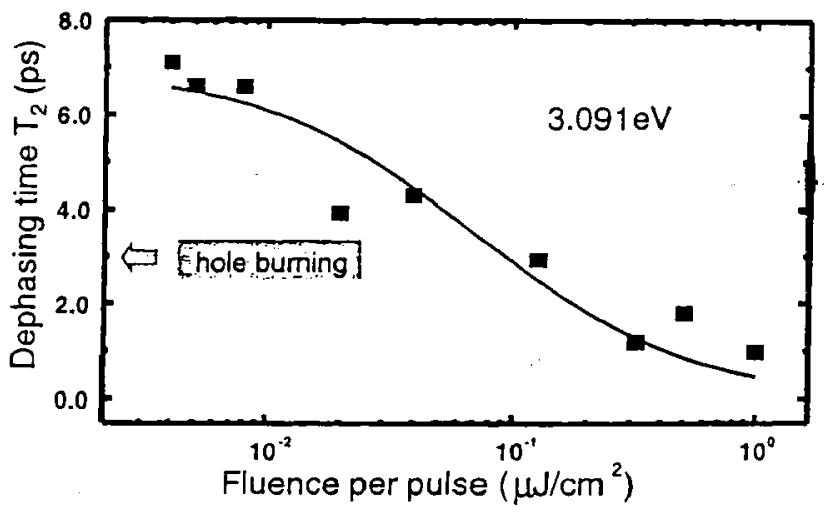

Figure 49 : Temps de dêcroissance du signal en fonction de l'intensité d'excitation à $400 \mathrm{~nm}(3,091 \mathrm{eV})$ et $5 \mathrm{~K}$.

Nous indiquons aussi dans la figure 49 la valeur de $T_{2}$ obtenue par des mesures de "spectral hole burning", qui est d'un facteur deux fois plus petit que la valeur trouvée par mélange à quatre ondes. Cette différence est systématique si l'on mesure à des énergies de photons d'excitation différentes. Ceci est dû aux techniques que l'on emploie : dans le cas du mélange d'onde, on détermine une valeur moyenne de $\mathrm{T}_{2}$ qui dépend faiblement de l'énergie de photon [45]. Dans le cas du "hole burning" les impulsions laser sont si longues que la distribution des charges dans la matrice peut se réarranger en déplaçant la fréquence de résonance de l'oscillateur. On en détermine donc une valeur de largeur spectrale moyennée en temps (" spectral diffusion ").

Si l'on change l'énergie de photon, à plus grandes énergies que celle de la figure 48 des oscillateurs de 250 fs de période apparaissent (figure 50). Il s'agit des battements quantiques qui sont observés quand différentes transitions cohérentes partagent un même état. Ici, on peut exciter un ou deux excitons dans le même nanocristal, ce qui donne lieu aux battements quantiques à des énergies de photons supérieures à celles de l'exciton.
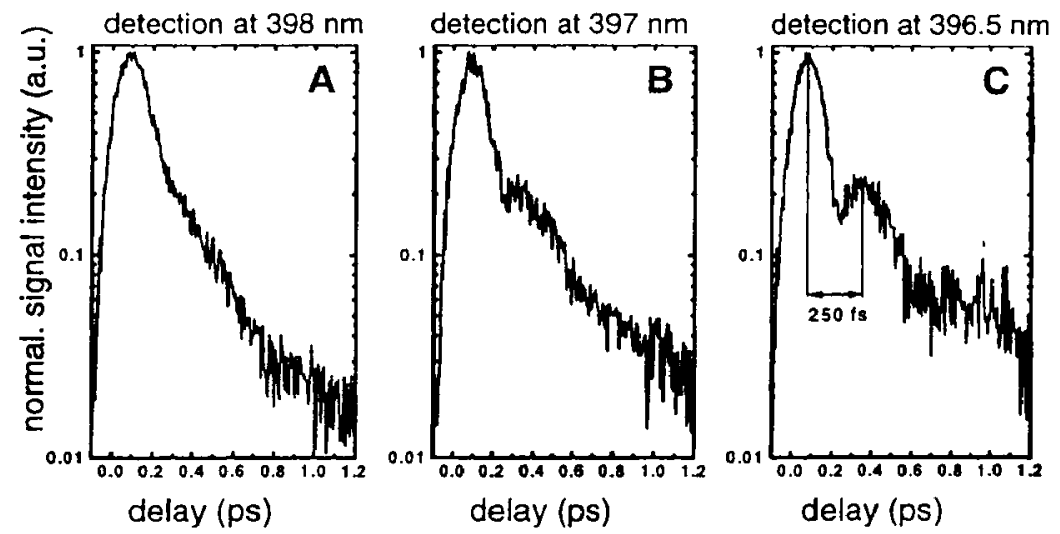

Figure 50 : Décroissances typiques du signal de mélange à quatre ondes aux longueurs d'onde d'excitation de $398 ; 397$ et $396.5 \mathrm{~nm}$. 


\subsection{Expériences à trois faisceaux résolues en temps}

Nous présentons ici la méthode de mesure du temps de vie et du coefficient de diffusion spatiale des porteurs photo-créés dans nos échantillons. Cette mesure se fait par mélange à quatre ondes dégénérées, dans une configuration à trois faisceaux. Dans cette configuration, le vecteur d'onde $\vec{k}_{3}$ du faisceau qui se diffracte sur le réseau est différent de ceux des faisceaux pompe $\mathrm{P}_{1}$ et $\mathrm{P}_{2}$.

Comme le montre la figure 51, trois faisceaux sont incidents sur l'échantillon. Il est donc possible de former trois réseaux distincts :

- avec les faisceaux de vecteurs d'onde $\vec{k}_{1}$ et $\vec{k}_{2}$, de pas $\vec{Q}_{1}=\vec{k}_{2}-\vec{k}_{1}$,

- avec les faisceaux de vecteurs d'onde $\vec{k}_{1}$ et $\vec{k}_{3}$, de pas $\vec{Q}_{2}=\vec{k}_{3}-\vec{k}_{1}$,

- avec les faisceaux de vecteurs d'onde $\vec{k}_{2}$ et $\vec{k}_{3}$, de pas $\vec{Q}_{3}=\vec{k}_{3}-\vec{k}_{2}$.

Chaque faisceau peut alors se diffracter sur chacun des réseaux.

Les faisceaux $P_{1}$ et $P_{2}$, arrivent en coüncidence temporelle sur l'échantillon et peuvent donc créer une modulation des populations des quasi-particules dont nous voulons tester l'évolution en temps.

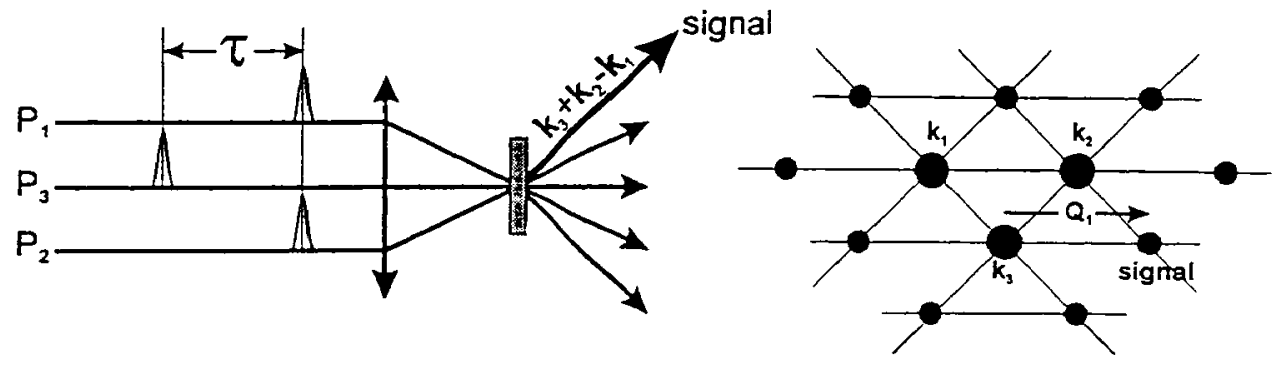

Figure 51 : Mélange à quatre ondes dégénérées, dans une configuration à trois faisceaux, destinée à la mesure du temps de vie et du coefficient de diffusion.
Figure 52 : Image formée sur un écran placé après l'échantillon, dans une configuration à trois faisceaux

Le signal que l'on étudie est la diffraction à l'ordre +1 (éventuellement -1 ) du faisceau $\mathbf{P}_{3}$ de vecteur d'onde $\vec{k}_{3}$, sur le réseau de pas $\vec{Q}_{1}=\vec{k}_{2}-\vec{k}_{1}$. Si $\vec{k}_{3}$ est dans le plan défini par $\vec{k}_{1}$ et $\vec{k}_{2}$, sa direction de propagation $\vec{k}_{3}+\vec{k}_{2}-\vec{k}_{1}$ reste dans ce plan et est confondue avec la diffraction d'autres faisceaux incidents sur les différents réseaux induits. Ainsi, afin de séparer le signal qui nous intéresse de ces autres contributions, on choisit de ne pas faire propager le faisceau $P_{3}$ dans le plan formé par les faisceau $P_{1}$ et $P_{2}$, mais dans une direction inclinée par rapport à celui-ci. La figure 52 est celle que l'on obtiendrait en plaçant un écran derrière l'échantillon, dans cette configuration.

Cet arrangement expérimental permet de séparer le signal qui nous intéresse des autres contributions. De plus, le signal se trouve plus éloigné des autres taches de diffraction ainsi que des transmissions des faisceaux incidents : le bruit éventuellement engendré par la 
diffusion de ces faisceaux intenses et détecté dans la direction du signal s'en trouve diminué.

La dynamique du signal de diffraction de $P_{3}$ contient une information relative au temps de vie des porteurs, puisque la recombinaison des porteurs créés par $P_{1}$ et $P_{2}$ détruit le réseau et empêche la génération d'un signal. Nous montrons maintenant comment la mesure de l'intensité du faisceau diffracté en fonction du retard entre les impulsions $P_{3}$ d'une part et celles de $P_{1}$ et $P_{2}$ d'autre part permettent de déterminer le temps de vie et le coefficient de diffusion des porteurs si elles sont effectuées pour plusieurs pas de réseau.

Nous négligeons dans la suite les effets cohérents discutés auparavant, et ne considérons que les effets dus à une modulation spatiale de la densité de porteurs. Notons toutefois que les “artefacts cohérents" peuvent souvent être éliminés expérimentalement par la configuration des polarisations que nous utilisons, par exemple une polarisation linéaire $\vec{P}_{1} / / \vec{P}_{2}$ et $\vec{P}_{3} \perp \vec{P}_{1}$.

L'évolution spatio-temporelle de la densité de porteurs excités $\mathrm{N}(\mathrm{x}, \mathrm{z}, \mathrm{t})$ peut être décrite par l'équation :

$$
\frac{\partial N(x, z, t,)}{\partial t}=G(x, z, t)-\frac{N(x, z, t)}{\tau(N)}+\nabla[D \nabla N(x, z, t)]
$$

où $G(x, z, t)$ est le terme de création des porteurs

$\tau(\mathrm{N})$ est le temps de recombinaison des porteurs, qui peut dépendre de $\mathrm{N}$,

D est le coefficient de diffusion ambipolaire.

Plusieurs approximations permettent de simplifier cette écriture :

- Dans les semi conducteurs, le taux de recombinaison $N / \tau$ est fortement dépendant de la densité de porteurs et peut être développé en une série de Taylor :

$$
\mathrm{N} / \tau=\mathrm{AN}+\mathrm{BN}^{2}+\mathrm{CN}^{3}+\ldots
$$

où $\quad A=1 / T_{1}$ correspond à la recombinaison linéaire,

B correspond à la recombinaison radiative bimoléculaire,

$C$ décrit la recombinaison Auger (recombinaison avec transfert de l'énergie d'un électron et d'un trou à un autre porteur).

De grandes intensités d'excitation favorisent ces processus non linéaires de recombinaison, et l'équation (79) devient alors particulièrement difficile à résoudre. Pour éviter ces complications, nous nous plaçons dans le cas de faibles intensités d'excitation, et considérons $\tau$ comme indépendant de la densité $\mathrm{N}(\mathrm{x}, \mathrm{z}, \mathrm{t})$.

- Nous ne considérons que le problème à une dimension, en supposant que la densité de porteurs ne varie que suivant la cordonnée $x$, direction du réseau. On a alors $N(x, z, t)=N(x, t)$. Ceci suppose l'épaisseur de l'échantillon petite devant le pas de réseau $\Lambda$ et une excitation homogène suivant $z$, c'est-à-dire dans l'épaisseur de l'échantillon.

Le coefficient D est un coefficient de diffusion ambipolaire, ce qui signifie que électrons et trous diffusent ensemble et qu'aucune charge d'espace ne se développe dans le volume de l'échantillon. À faible densité de porteurs et dans le cas unidimensionnel, on a (loi de Fick) :

$$
\nabla[D \nabla N(x, t)]=D \frac{\partial^{2} N(x, t)}{\partial x^{2}}
$$


Si l'on considère une création instantanée des porteurs à $t=0$ par une impulsion de Dirac, l'équation (79) devient dans ces conditions :

$$
\frac{\partial N(x, t)}{\partial t}=K I_{0}\left[1+\cos \left(\frac{2 \pi x}{\Lambda}\right)\right]-\frac{N(x, t)}{T_{1}}+D \frac{\partial^{2} N(x, t)}{\partial x^{2}}
$$

$\mathrm{K}$ étant un coefficient décrivant les pertes dues aux réflexions à la surface de l'échantillon. La solution de l'équation (82) est donnée par [69] :

$$
N(x, t)=N_{0}\left[1+e^{-t / \tau_{D}} \cos \left(\frac{2 \pi x}{\Lambda}\right)\right] e^{-t / T_{1}}
$$

où $\mathrm{N}_{0}$ est la densité de porteurs initiale et $\tau_{\mathrm{D}}$ le temps de diffusion des porteurs :

$$
\tau_{D}=\frac{\Lambda^{2}}{4 \pi^{2} D}
$$

Le déclin de la modulation spatiale est ainsi caractérisé par le temps $\tau_{\mathrm{g}}$ :

$$
\frac{1}{\tau_{8}}=\frac{4 \pi^{2} D}{\Lambda^{2}}+\frac{1}{T_{1}}
$$

Ce temps de déclin du réseau est mesuré en observant la dépendance temporelle de la diffraction d'un faisceau test $P_{3}$ : l'efficacité de diffraction $\eta$ est mesurée en fonction du retard $\Delta$ t entre l'impulsion de $P_{3}$ et celles de $P_{1}$ et $P_{2}$ qui coincident temporellement. Dans le cas de faibles intensités d'excitation, l'efficacité de diffraction dépend de façon quadratique de la variation $\Delta \tilde{\boldsymbol{n}}$ d'indice complexe et de la densité $\mathrm{N}$ de porteurs :

$$
\eta(\Delta t) \propto|\Delta \tilde{n}|^{2} \propto N^{2}
$$

C'est pourquoi le temps $\tau$ de déclin mesuré expérimentalement vaut la moitié du temps de déclin du réseau :

$$
\tau=\frac{\tau_{g}}{2}
$$

L'équation (85) sépare deux contributions distinctes au déclin du réseau : celui-ci s'efface dans le temps du fait de la diffusion des porteurs (premier terme de l'équation), mais aussi du fait de la recombinaison de ces porteurs (deuxième terme). Expérimentalement, il est possible de séparer ces deux contributions en réitérant la mesure de $\tau_{\mathrm{g}}$ pour différents pas du réseau de diffraction (en modifiant l'angle $\theta$ d'intersection entre les faisceaux $P_{1}$ et $P_{2}$ ). La dépendance de $1 / \tau_{\mathrm{g}}$ en fonction de $4 \pi^{2} / \Lambda^{2}$ est alors linéaire, de pente donnée par le coefficient de diffusion $\mathrm{D}$ et d'ordonnée à l'origine $1 / \mathrm{T}_{1}$.

Un exemple est montré sur la figure 53 où on a excité à température ambiante CdS autour de la résonance excitonique. Les différentes courbes correspondent à des pas de réseau différents [70]. Il faut noter que la décroissance est exponentielle mais dépend de l'échantillon [71] parce que la durée de vie des paires électron-trous peut être limitée par des effets extrinsèques (des centres de recombinaison, etc.). De plus, comme mentionné avant, elle dépend de l'intensité d'excitation [72]. 


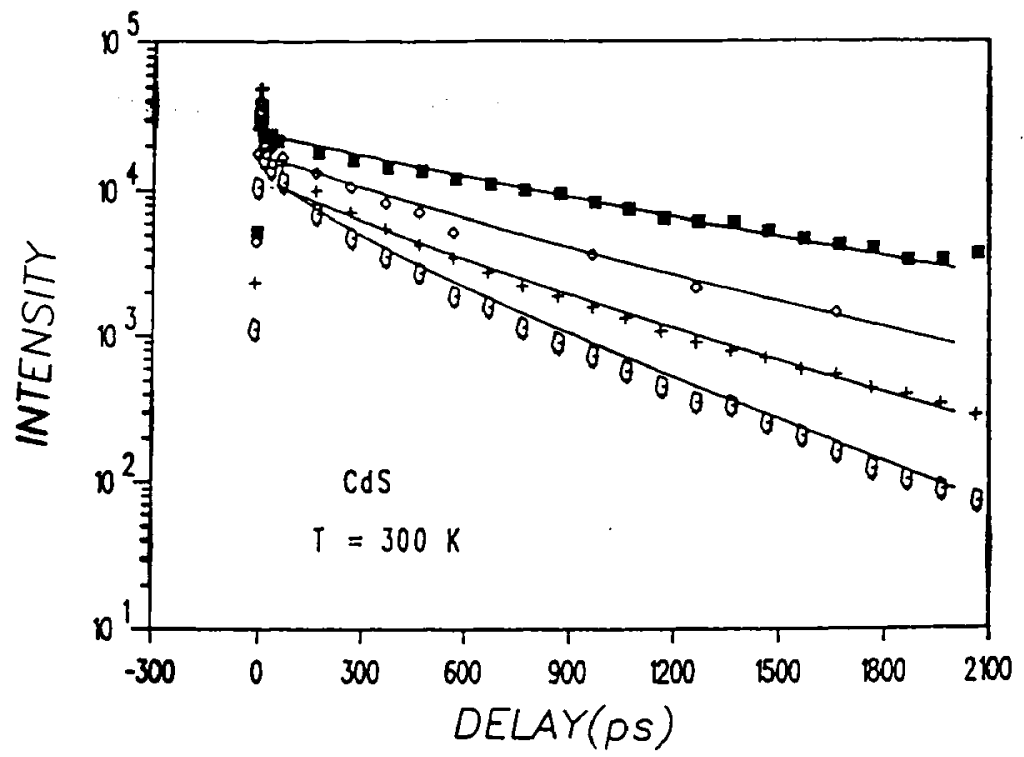

Figure 53 : Intensité du signal en fonction du retard entre $P_{3}$ et $P_{1}$ pour différentes valeurs de $\Lambda$. $(\Lambda=8,5,4,3 \mu \mathrm{m}$ du haut vers le bas).

Dans CdS, le coefficient de diffusion ambipolaire D est anisotrope : pour des paires électron-trous il est donné par

$$
D=\frac{D_{e} D_{h}}{D_{e}+D_{h}}
$$

où, par la relation d'Einstein

$$
D_{e, h}=\frac{\mu_{e, h} k_{B} T}{e}
$$

$D_{e, h}$ sont les coefficients de diffusion des électrons et trous, respectivement, $\mu_{e, h}$ est leur mobilité et $\mathrm{k}_{\mathrm{B}}$ la constante de Boltzmann. Dans un simple modèle de Drude

$$
\mu_{e, h}=\frac{e \tau_{\text {coll }}}{m_{e, h}}
$$

où $\tau_{\text {coll }}$ représente le temps moyen entre deux collisions successives et $m_{e, h}$ est la masse effective des électrons et trous. La masse effective de la bande de valence A de CdS est anisotrope. Elle vaut $[1,73]$

$$
m_{h \perp}=0,7 \mathrm{~m}_{0} \text { et } \mathrm{m}_{\mathrm{h} /}=2,5 \mathrm{~m}_{0}
$$

pour $\vec{k} \perp \vec{c}$ et $\vec{k} / / \vec{c}$ où $\vec{c}$ représente l'axe cristallin de CdS. La masse effective de la bande de conduction $\mathrm{m}_{\mathrm{e}}$ par contre est isotrope.

Dans une autre série d'expériences [71] nous avons testé cet effet à $\mathrm{T}=300 \mathrm{~K}$ en excitant $\mathrm{CdS}$ près de la transition bande à bande $(\hbar \omega=2,475 \mathrm{eV})$. Le résultat est montré sur la figure 54 où l'anisotropie de la constante de diffusion est entièrement expliquée par l'anisotropie de la bande de valence. La constante de diffusion D diminue et devient isotrope 
si l'on excite à $\hbar \omega=2,41 \mathrm{eV}$. Dans ce cas (Fig.55), on ne créé plus de paires électrons trous libres mais localisés. La diffusion est ici entièrement due au "hopping" entre ces états localisés. On remarque aussi que la durée de vie des excitations élémentaires (donnée par les valeurs d'intersection des droites avec l'ordonnée) est dans tous les cas de l'ordre de 10-20 ns.

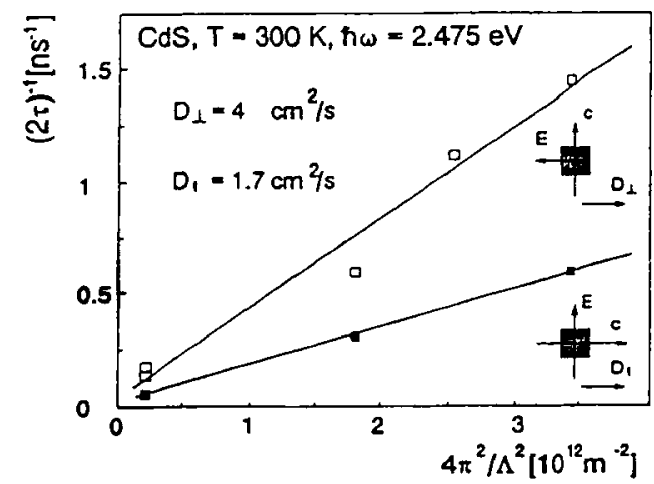

Figure 54 : Temps de déclin du signal en fonction de $1 / \Lambda$ pour deux orientations de l'échantillon différentes

L'excitation est à $\hbar \omega=2.475 \mathrm{eV}$ avec $\vec{E} \perp \vec{c} ;$ la diffusion $\vec{D} / / \vec{C}$ (carrés pleins) et $\vec{D} \perp \vec{c}$ (carrés ouverts) [71].

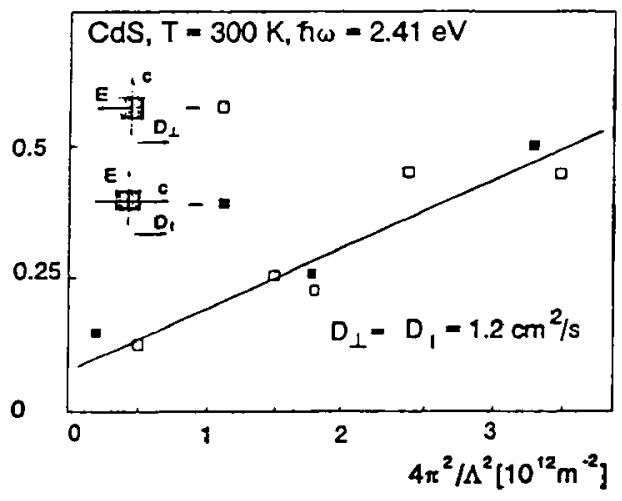

Figure 55 : Comme Fig. 54 mais pour une excitation à $\hbar \omega=2.41 \mathrm{eV}[71]$.

On peut noter que des expériences similaires ont été effectuées sur les nanocristaux de CdS dans une matrice de verre [74] préparée par la méthode sol-gel. Dans ce cas, à faible intensité, une diffusion n'a pas été observée. La dynamique du signal est indépendante du pas du réseau, mais montre une décroissance biexponentielle. Ceci est la signature d'un système où plusieurs états de durées de vie différentes interviennent et donnent lieu à des nonlinéarités optiques.

\subsection{Mélange à quatre ondes non dégénérées}

La détermination du temps de déphasage est envisageable dans l'espace des temps, comme nous venons de le voir. Cette technique nécessite cependant de disposer d'impulsions laser suffisamment brèves pour pouvoir résoudre $T_{2}$. Dans la plupart des cas, ceci n'est devenu possible qu'avec l'avènement de sources laser femtosecondes. Il faut d'autre part que ces impulsions soient accordables à la longueur d'onde pour laquelle l'on souhaite effectuer la mesure (souvent en bord de bande pour les semi-conducteurs). Une alternative à ces exigences est d'effectuer la mesure du temps de déphasage dans l'espace des fréquences. Nous présentons ici une technique : le mélange à quatre ondes non dégénérées.

Cette technique consiste à faire interférer à la surface de l'échantillon deux impulsions laser en coïncidence temporelle, de fréquence $\omega_{1}$ et $\omega_{2}$ proches mais différentes [75]. Il est habituel de disposer d'une source laser accordable autour de l'émission fixe de l'autre. La détermination de $T_{1}$ et $T_{2}$ repose sur la mesure de l'intensité du signal diffracté en fonction de la différence $\omega_{d}$ entre les fréquences des lasers excitateurs : $\omega_{d}=\omega_{1}-\omega_{2}$. 
L'intensité I résultante des battements entre deux ondes planes de pulsation $\omega_{1}$ et $\omega_{2}$, d'amplitudes $E_{1}$ et $E_{2}$ et de vecteurs d'onde $\vec{k}_{1}$ et $\vec{k}_{2}$ s'écrit :

$$
I \propto \frac{1}{2}\left|E_{1}\right|^{2}+\frac{1}{2}\left|E_{2}\right|^{2}+E_{1} E_{2} \cos \left[\left(\omega_{1}-\omega_{2}\right) t+\left(\vec{k}_{1}-\vec{k}_{2}\right) \vec{r}\right]
$$

Le réseau qui est inscrit est un réseau mobile, qui se déplace parallèlement à la surface de l'échantillon à la vitesse $\left(\omega_{1}-\omega_{2}\right) /\left(\mathbf{k}_{1}-\mathbf{k}_{2}\right)$. On parle plutôt de battements entre les deux fréquences que d'interférences, puisque l'intensité résultante à la surface de l'échantillon est modulée non seulement spatialement mais aussi temporellement.

Le signal de diffraction est émis dans la direction $2 \vec{k}_{2}-\vec{k}_{1}$, à une fréquence $\omega_{3}=2 \omega_{2}-\omega_{1}$ différente de celle des impulsions pompes. Ainsi, lorsque la détection se fait avec une résolution spectrale, le rapport signal/bruit attendu pour ces mesures est très grand.

La modélisation mathématique relative à cette technique est basée sur le formalisme de la matrice densité. Elle passe par la résolution de son équation d'évolution par la méthode des perturbations, dans le cas d'un système à deux niveaux et dans l'approximation de l'onde tournante. On obtient de cette façon la polarisation du système. La polarisation macroscopique est obtenue en intégrant la polarisation microscopique sur l'élargissement inhomogène des raies. Dans le cas d'un élargissement inhomogène $\Delta \omega_{i}$ satisfaisant à la condition :

$$
\Delta \omega_{i} \gg\left(T_{2}^{-1}, T_{1}^{-1},\left|\omega_{2}-\omega_{1}\right|\right)
$$

on obtient :

$$
P^{(3)}\left(\omega_{3}\right)=\chi^{(3)} E_{1}^{*} E_{2}^{2} e^{-i \omega_{3} t}+c c
$$

avec :

$$
\begin{gathered}
\chi^{(3)}=\chi_{R}^{(3)}+\chi_{N R}^{(3)} \\
\chi_{R}^{(3)}=\frac{i K_{3}}{\left(1+i \omega_{d} T_{1}\right)\left(1+i \omega_{d} T_{2}\right)}
\end{gathered}
$$

$\chi_{R}^{(3)}$ et $\chi_{N R}^{(3)}$ sont les susceptibilités non linéaires d'ordre 3 associées respectivement à la transition résonante (celle prise en compte dans le système à deux niveaux) et aux autres transitions (non résonantes). Le terme $\mathbf{K}_{3}$ peut être considéré comme constant sur l'intervalle de variation de $\omega_{\mathrm{d}}$. L'intensité du signal de sortie, à la fréquence $\omega_{3}$ étant proportionnelle au module au carré de la susceptibilité non linéaire d'ordre 3, on peut écrire :

$$
I_{S} \propto\left|\chi^{(3)}\right|^{2}=\left|\frac{i K_{3}}{\left(1+i \omega_{d} T_{1}\right)\left(1+i \omega_{d} T_{2}\right)}+\chi_{N R}^{(3)}\right|^{2}
$$

On voit ainsi que sa mesure donne directement des informations à la fois sur le temps de relaxation $\mathrm{T}_{1}$ et sur le temps de déphasage $\mathrm{T}_{2}$. Le terme $\chi_{N R}^{(3)}$ est également considéré comme constant sur l'intervalle de variation de $\omega_{\mathrm{d}}$. Cela a pour effet de faire interférer les termes résonants et non résonants et on attend pour cette raison une forme asymétrique du signal [76], selon le signe de $\omega_{\mathrm{d}}$. 
Dans le cas des nanocristaux de CdSe dans un verre de borosilicate, le terme non résonant n'avait pas d'importance [77]. Comme nous le voyons sur la figure 56, qui montre l'intensité du signal en fonction de $\hbar\left(\omega_{2}-\omega_{1}\right)$ on couvre une grande plage spectrale et la variation de l'intensité peut être mesurée sur cinq ordres de grandeur si le terme non résonant est faible. En utilisant le modèle [75] on trouve des valeurs de $T_{1}=10 \pm 1 \mathrm{ps}, \mathrm{T}_{2}=100 \pm 20 \mathrm{fs}$ pour l'échantillon vierge et $T_{1}=0,6 \pm 0,1$ ps et $T_{2}=30 \pm 20 \mathrm{fs}$ pour l'échantillon noirci. L'origine de ce noircissement est discuté dans le cours de D. Ricard de cet ouvrage. De plus, la résonance Raman-Stokes apparaît comme maximum secondaire. Elle ne pouvait pas être traitée dans le cadre de la théorie [75].

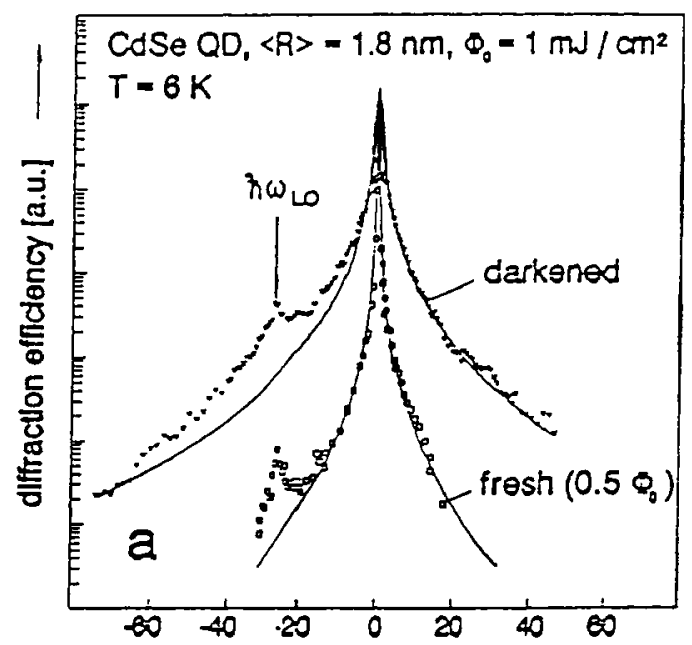

Figure 56 : Mélange à quatre ondes non dégénérées dans des nanocristaux de CdSe avec des échantillons vierges et noircis par excitation laser [76]

Dans le cas des nanocristaux, la théorie [75] peut être appliquée parce que la raie s'est élargie d'une manière inhomogène. Outre la détermination des temps de relaxation $T_{1}$ et $T_{2}$. on peut aussi utiliser cette même technique du mélange à quatre ondes non dégénérées pour la détermination de l'accord de phase dans un processus de collision similaire à la diffusion Hyper-Raman. On peut ainsi déterminer la déformation de la relation de dispersion, induite par la haute intensité d'excitation.

La configuration géométrique est résumée pour les processus sur la figure 57 . Dans le processus de diffusion Hyper-Raman seul le faisceau pompe est présent. Ill excite un état intermédiaire dont la recombinaison a lieu avec conservation d'énergie et de la quantité de mouvement :

$$
\begin{aligned}
& 2 \hbar \omega_{P}=E(q)+E(K) \\
& 2 \vec{k}_{P}=\vec{q}+\vec{K}
\end{aligned}
$$

Ceci est un processus spontané. 


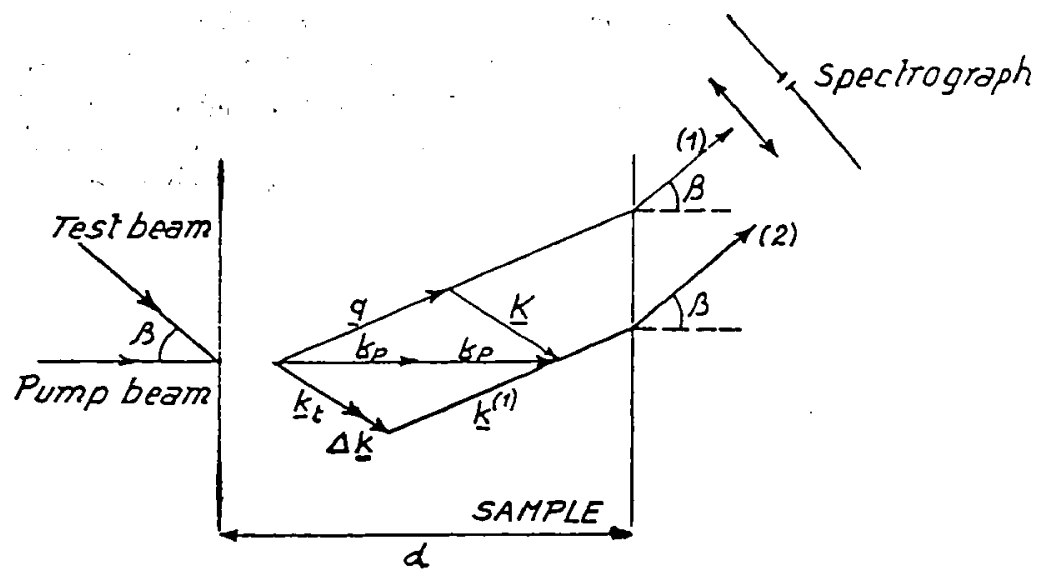

Figure 57 : Mélange à quatre ondes non dégénérées et diffusion Hyper Rarnan.

Dans le cas de mélange à quatre ondes, le faisceau test de pulsation $\omega_{1}$ et de vecteur d'onde $\vec{k}_{t}$ induit la recombinaison de l'état intermédiaire et donne lieu à l'émission d'un signal de pulsation $\omega^{(1)}$ et de vecteur d'onde $\vec{k}^{(1)}$ tel que

$$
\begin{aligned}
& 2 \hbar \omega_{P}=\hbar \omega_{t}+\hbar \omega^{(1)} \\
& 2 \vec{k}_{P}=\vec{k}_{t}+\vec{k}^{(1)}+\Delta \vec{K}
\end{aligned}
$$

Ce processus induit a lieu avec conservation d'énergie mais fait intervenir un désaccord de phase $\Delta \vec{K}$ :

$$
\Delta \vec{K}=2 \vec{k}_{p}-\vec{k}_{s}-\vec{k}^{(1)}
$$

Il devient nul lorsque $\vec{k}_{t}=\vec{K}$ et en conséquence $\vec{k}^{(1)}=\vec{q}$ ou l'inverse.

D'après l'équation (65) l'intensité du signal varie comme :

$$
I^{(1)} \propto\left|\chi^{(3)}\right|^{2} I_{p}^{2} I_{t} d^{2}\left(\frac{\sin (\Delta K d / 2)}{\Delta K d / 2}\right)^{2}
$$

Si l'on garde la configuration, les intensités et $\hbar \omega_{\mathrm{P}}$ constants et que l'on ne fasse varier que $\hbar \omega_{t}, \Delta \mathrm{K}$ varie rapidement et un spectre d'excitation de $\mathrm{I}^{(1)}$ montre un maximum prononcé quand $\Delta \vec{K}=0$. Comme le montre la figure 58 pour $\mathrm{CuCl}$ à $4 \mathrm{~K}$ en choisissant $\beta=11^{\circ}$ sa position spectrale coïncide bien avec la position spectrale des raies Hyper-Raman [78] (indiqué par les flèches) mesurées dans la même expérience en coupant le faisceau test. L'intensité de $\mathrm{I}^{(1)}$ est, par contre, plusieurs ordres de grandeurs plus importante que celle des raies Hyper-Raman. Nous avons donc pu étudier, grâce à cette technique [79] la variation de la zone induite par laser montrée sur la figure 20 en fonction de son intensité. Dans $\mathrm{CuCl}$, nous avons choisi une situation angulaire $\left(\beta=10^{\circ}\right)$ et une énergie de photon $\hbar \omega_{\mathrm{p}}=3.1845 \mathrm{eV}$ telle que $\hbar \omega^{(1)}$ se trouve dans la région de dédoublement des raies Hyper-Raman. La figure 59 montre le résultat où $\hbar \omega$ indique la position du maximum du spectre d'excitation et la flèche 
la position spectrale de la raie de Hyper-Raman à faible intensité. Les courbes en pointillé donnent le résultat calculé pour les positions des raies dédoublées discutées avant. On voit bien que, pour une densité $n_{p}<2.10^{13} \mathrm{~cm}^{-3}$ photons du faisceau pompe, une seule solution des équations (30) et (31) existe et que pour $n_{p} \geq 10^{15} \mathrm{~cm}^{-3}$ les effets de saturation interviennent. Pour ajuster la théorie aux points expérimentaux ils faudrait augmenter largement les constantes d'amortissement intervenant dans la matrice densité.

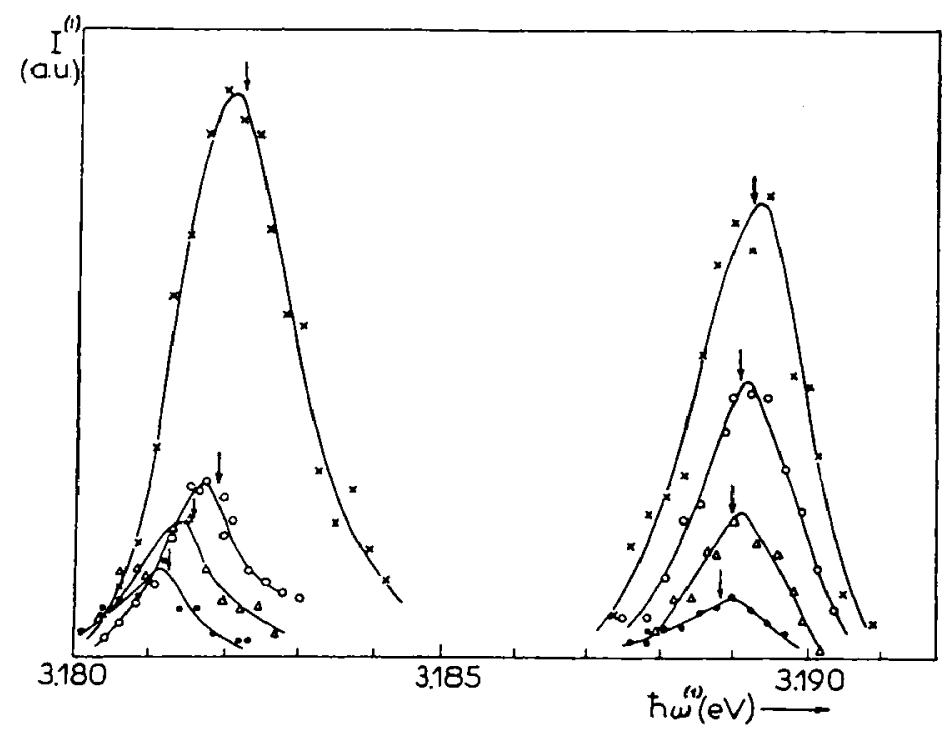

Figure 58 : Intensité du signal $\mathrm{I}^{(1)}$ en fonction de $\hbar \omega^{(1)}=2 \hbar \omega_{\mathrm{p}}-\hbar \omega_{\mathrm{i}}$ pour différentes énergies de photon $\hbar \omega_{\mathrm{p}}=(3,1858 ; 3,1856 ; 3,1854$ et $3,1851 \mathrm{eV}$ du haut vers le bas $)$. Les flèches indiquent les positions spectrales des raies Hyper-Raman pour les mêmes intensités d'excitation.

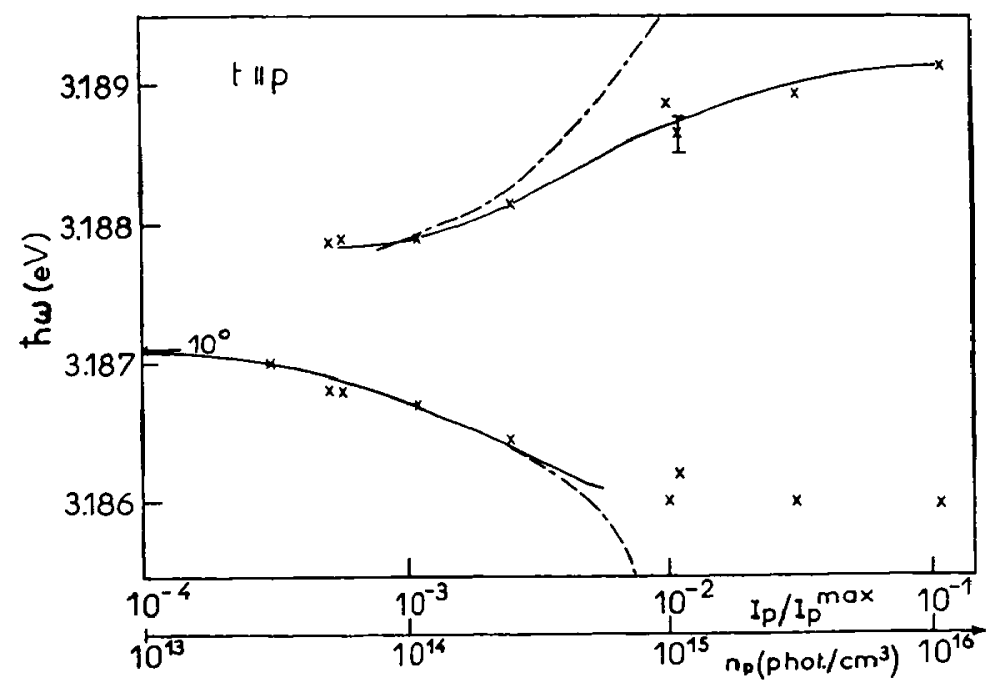

Figure 59 : Position des maxima des spectres d'excitation en fonction de l'intensité du faisceau pompe qui induit la renormalisation de la relation de dispersion. 
Ces mesures ont été effectuées en utilisant des polarisations des faisceaux test et pompe linéaires et parallèles. Comme discuté avant, pour des polarisations croisées, l'anomalie due à l'absorption à deux photons disparaît et seule celle due à l'absorption induite reste. Elle donne lieu à un déplacement global de la relation de dispersion dans la région spectrale étudiée ici [79]. Cet effet a aussi pu être étudié en fonction de l'intensité d'excitation.

\section{CONCLUSION}

Dans cet article j'ai passé en revue différentes techniques utilisées en spectroscopie non linéaire. Je me suis largement appuyé sur des exemples venant du domaine de physique des semi-conducteurs et des résultats obtenus dans notre équipe de recherche. Voulant être illustratif, je n'ai pas cité le grand nombre de résultats originaux et importants obtenus par d'autres équipes de recherche et je prie le lecteur de m'en excuser. Une bibliographie plus équilibrée est présentée dans les différents articles de revue et livres cités ici.

J'espère avoir pu montrer la richesse que présente la spectroscopie non linéaire. Il ne faut pas oublier, par contre, que chaque technique ne donne que des renseignements ponctuels sur les propriétés d'un matériau et qu'il faut utiliser le plus grand nombre de techniques pour bien le caractériser et faire la part entre ses propriétés extrinsèques et intrinsèques.

\section{Références}

[1] Hönerlage B., Lévy R., Grun J.B., Klingshirn C. et Bohnert K., Phys. Rep. 124 (1985) 161 et références citées.

[2] Klingshirn C.: "Semiconductor Optics", Springer Verlag, Heidelberg (1995) et références citées.

[3] Born M. etWolf E. : “Principles of Optics ”, Pergamon, Oxford (1977).

[4] Masumoto Y., Unuma Y., Tanaka Y. et Shionoya S., J. Phys. Soc. Japan 47 (1979) 1844 ;

Masumoto Y., Unuma Y. et Shionoya S., J. Phys. Soc. Japan 49 Suppl. A (1980) 393.

[5] Reimann K. et Rübenacke St., Phys. Rev. B 49 (1994) 11021.

[6] Fröhlich D., Mohler E. et Wiesner P., Phys. Rev. Lett. 26 (1971) 554.

Staginnus B., Fröhlich D. et Lapes T., Rev. Sci. Instr. 39 (1968) 1129.

[7] Fröhlich D. et Nieswand W., Phil. Mag 70 (1994) 321.

[8] Grun J.B., Hönerlage B. et Lévy R. dans Excitons, E.I. Rashba \& M.D. Sturge, éditions North Holland Publish. Comp. Amsterdam, (1982) p. 459.

[9] Vu Duy Phach, Bivas A., Hönerlage B. et Grun J.B., Phys. Status Solidi (b) 86 (1978) 159.

[10] Hönerlage B., Vu Duy Phach, Bivas A. et Ostertag E., Phys. Status Solidi (b) 83 (1977) 101.

[11] Itoh T., Suzuki T. et Ueta M., J. Phys. Soc. Japan 42 (1977) 1069.

.[12] Hönerlage B., Bivas A. et Vu Duy Phach, Phys. Rev. Lett. 41 (1978) 49.

[13] Itoh T. et Suzuki T., J. Phys. Soc. Japan 45 (1978) 1939.

[14] Nagasawa N., Mita T. et Ueta M., J. Phys. Soc. Japan 41 (1976) 929.

[15] Ostertag E., Phys. Rev. Lett. 45 (1980) 372.

[16] Mita T., Satomé K. et Ueta M., Solid State Commun. 33 (1980) 1135. 
[17] Hönerlage B., Rössler U., Vu Duy Phach, Bivas A. et Grun J.B., Phys. Rev. B 22 (1980) 797. Bivas A., Vu Duy Phach, Hönerlage B., Rössler U. et Grun J.B., Phys. Rev. B 20 (1979) 3442.

[18] Vu Duy Phach et Lévy R., Solid State Commun. 29 (1979) 247.

[19] Lévy R., Hönerlage B. et Grun J.B., Phys. Rev. Let. 44 (1980) 1355.

[20] Hönerlage B., Lévy R. et Grun J.B., Phys. Rev. B 24 (1981) 3211.

[21] Bigot J.Y. et Hönerlage B., Phys. Status Solidi (b) 121 (1983) 649.

[22] Hönerlage B. et Bigot J.Y., Phys. Status Solidi (b) 123 (1984) 201.

[23] Hönerlage B. et Bigot J.Y., Phys. Status Solidi (b) 124 (1984) 221.

[24] Risken H., et Vollmer H.D., Z. Phys. B, 39 (1980) 339.

[25] Grun J.B., Hönerlage B. et Lévy R., Solid State Commun. 46 (1983) 51.

[26] Lévy R., Hönerlage B. et. Grun J.B., Helv. Acta Physica, 58 (1985) 252.

[27] Itoh T., Suzuki T. et Ueta M., J. Phys. Soc. Japan, 44 (1978) 345.

[28] Itoh T. et Suzuki T., J. Phys. Soc. Japan, 45 (1978) 1939.

[29] Lévy R., Hönerlage B. et Grun J.B. dans "Optical Nonlinearities and Instabilities in Semiconductors, H. Haug editeur, Academic Press, New York (1988)

[30] Lévy R., Hönerlage B. et Grun J.B., Solid State Commun 29 (1979) 103 ; Phys. Rev. B 19 (1979) 2326

[31] Lee M., Song O., Seo J., Kim D., Suh Y.D., Jin S.M. et Kim S.K., Chem. Phys. Lett. 196, (1992) 4325.

[32] Kost A., Tutt L., Klein M.B., Dougherty T.K. et Elias WE Opt. Lett. 18 (1993) 335.

[33] Schell, J. Brinkmann D., Ohlmann D., Hönerlage B., Lévy R., Joucla M., Rehspringer J.L., Serughetti J. et Bovier C. J. Chem. Phys. 108 (1998) 8599.

[34] Netiksis V., Hönerlage B., Weil R., Loison J.L.,. Grun J.B et Lévy R. J. Appl. Phys. 74 (1993) 5729.

[35] Benhmida M., Netiksis V., Robino M., Grun J.B., Petrauskas M. et Hönerlage B., J. Appl. Phys. 80 (1996) 4632.

[36] Pelant I., Ohlmann D., Cregut O., Granger R., Triboulet R. et Hönerlage B., Solid. State Commun. 85 (1993) 815.

[37] Ohlmann D., Cregut O., Pelant I., Granger R., Triboulet R. et Hönerlage B., J. Lumin. 54, (1993) 357.

[38] Ohlmann D., Hönerlage B., Pelant I., Weil R. et Muranevich E, Nonlinear Optics 5 (1993) 235.

[39] Sheik-Bahae M., Said A.A., Wei T-H, Hagan D.J., Van Stryland E.W., IEEE Quant. Electron. 26 (1990) 760.

[40] Yaviv A., “ Quantum Electronics ”, Wiley \& Sons, New York, (1975).

[41] Gilliot P., Merle J.C., Lévy R., M. Robino et Hönerlage B., Phys. Stat. Solidi (b) 153 (1989) 403

[42] Gilliot P., Moniatte J., Cregut O. et Lévy R., Phys. Stat. Solidi (b) 164 (1997) 441.

[43] Moniatte J., Gilliot P., Valenta J. et Ekimov A.I., Optical Materials 9 (1998) 516.

[44] Lévy R., Hönerlage B. et Gilliot P., Proc SPIE 3277 (1998) 71.

[45] Valenta J., Moniatte J., Gilliot P., Hönerlage B., Grun J.B., Lévy R. et Ekimov A.I., Phys. Rev. B, 57 (1998) 1774.

[46] Valenta J., Moniatte J., Gilliot P., Lévy R., Hönerlage B. et Ekimov A.I., Appl. Phys. Lett. 70 (1997) 680.

[47] Benoit à la Guillaume C., Debever J.M. et Salvan F., Phys. Rev. 177 (1969) 567.

[48] Magde D. et Mahr H., Phys. Rev. Lett., 24 (1970) 890. 
[49] Obayaski A. et. Gunshor R.L, Phys. Stat. Sol. (b), 159 (1990) 443.

[50] Hvam J.M., Phys. Stat. Sol. (b), 63 (1974) 511.

[51] Presser N., Kudlek J., Gutowski J., J. Lumin. 53 (1992) 435.

[52] Hönerlage B., Klingshirn C. et Grun J.B., Phys. Stat. Sol. (b) 78 (1976) 599.

[53] Haug H. et Koch S., Phys. Stat. Sol. (b) 82 (1977) 531.

[54] Klingshirn C., Maier W., Hönerlage B., Haug H. et Koch S.W., Solid State Electr. 21 (1978) 1357.

[55] Hönerlage B. et Rössler U., Molecular Spectro. of Dense Phase-Proc. 12th European Congress on Molecular Spectroscopy (1975). Elsevier, Amsterdam (1976) p. 105.

[56] Klingshirn C., Lévy R., Grun J.B. et Hönerlage B., Solid State Commun. 20 (1976) 413.

[57] Shaklee K.L., Leheny R.F. et Nahory R.E., Phys. Rev. Lett., 26 (1971) 888.

Shaklee K.L. et Leheny R.F., Appl. Phys. Lett., 18 (1971) 475.

[58] Shaklee K.L., Nahory R.E. et Leheny R.F., J. Lum., 7 (1973) 284.

[59] Valenta J., Guennani D., Manar A. et Hönerlage B., Solid State Comm. 98 (1996) 695.

[60] Vlasov Yu., Luterova K., Pelant I., Hönerlage B. et Astratov V.N., Appl. Phys. Lett 71 (1997) 1616.

[61] Tomashiunas R., Pelant I., Hönerlage B., Lévy R., Cloitre T., Aulombard R.L., Phys. Rev. B 57 (1998) 13077.

[62] Eichler H.J., Günter P. et Pohl D.W. “ Laser Induced Gratings " Springer Verlag, Berlin (1986).

[63] Maker P.D. et Terhune R.W., Phys. Rev. A, 137 (1965) 801.

[64] Yablonovitch E., Flytzanis C. et Bloembergen N., Phys. Rev. Letters 29 (1972) 865.

[65] Faller P., Netiksis V., Grun J.B. et Hönerlage B., J.Appl.Phys. 74 (1993) 2748

[66] Ghanassi M., Schame-Klein M.C., Hache F., Ekimov A.E., Ricard D. et Flytzanis C., Netiksis V., Grun J.B. et Hönerlage B., J. Appl. Phys. 74 (1993) 2748.Appl. Phys. Lett. 62 (1993) 78.

[67] Lévy R., Gomes M.J.M, Kippelen B. et Hönerlage B., Phys. Stat. Solidi (b) 159 (1990) 391.

[68] Lévy R., Hönerlage B. et Grun J.B., Phys. Stat. Solidi (b) 150 (1988) 825.

[69] Eichler H.J. et Massman F., J. Appl. Phys. 53 (1982) 3237.

[70] Kippelen B., Grun J.B., Hönerlage B. et Lévy R., JOSA B8 (1991) 2363.

[71] Riblet P., Spiegelberg Ch., Faller P., Gilliot P., Puls J., Henneberger F. et Hönerlage B., Optical Materials 3 (1994) 139.

[72] Hönerlage B., Optical Materials 1 (1992) 133 ; J. Luminescence 54 (1992) 113.

[73] Yu P.Y. et Evangelista F., Solid State Commun. 27 (1978) 87.

[74] Vanagas E., Moniatte J., Mazilu M., Riblet P., Hönerlage B., Juodkazis S., Paille F., Plenet J.C., Dumas J,. Petrauskas M. et Vaitkus J., J. Appl. Phys. 81 (1997) 3586.

[75] Yajima T. et Souma H., Phys. Rev. A, 17 (1978) 309.

[76] Tomashiunas R., Moniatte J., Pelant J., Gilliot P. et Hönerlage B., Appl. Phys. Lett. 86 (1996) 3296.

[77] Jungnickel V., Henneberger F., Riblet P. et Hönerlage B., Proc. Int. Conf. Opt. Properties of Nanostructures, Sendai (1994) ; Jap. Journ. Applied Phys. 34 (1995) 281.

[78] Hönerlage B., Lévy R. et Grun J.B., Optics Commun. 43 (1982) 443.

[79] Hönerlage B., J.Y. Bigot, Lévy R., Tomasini F. et Grun J.B., Solid State Commun. 48 (1983) 803. 